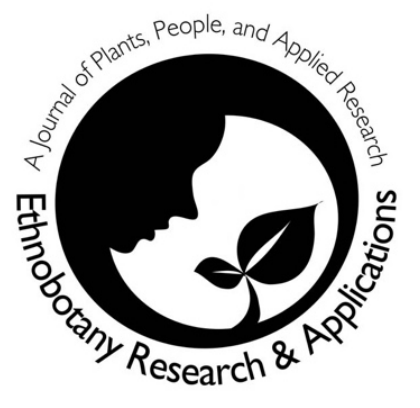

\title{
A Review of Ethnomedicinal Plant Resources in Southern Nigeria
}

\author{
K.C. Enebeli-Ekwutoziam, C.B. Aruah, B.O. Ogbonna, U.J. \\ Eze, F.S. Egedaye-Fubura, C.F. Nwankwo, I.N. Oliseyenum, \\ N.W. Udoha, T.N. Afuye, G.N. Asogwa, K.A. Chinweokwu, \\ F.O. Anenih, J.E. lyamu, I.D. Oboti, N. Nwaizu, J.I, Ajabor, \\ O.Y. Ozadibe, R.A. Otunla, Okoronkwo Bertha Francis and \\ Chukwu Miriam Orji
}

\begin{abstract}
Correspondence
K.C. Enebeli-Ekwutoziam ${ }^{1}$, C.B. Aruah ${ }^{1}$, B.O. Ogbonnaa ${ }^{1}$, U.J. Eze ${ }^{1}$, F.S. Egedeye-Fubara1, C.F. Nwankwo ${ }^{1}$, I.N.

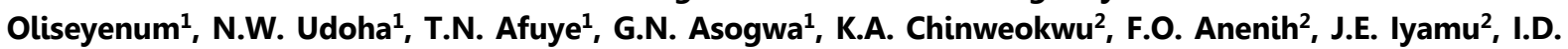
Oboti $^{2}$, N. Nwaizu ${ }^{2}$, J.I, Ajabor ${ }^{2}$, O.Y. Ozadibe ${ }^{2}$, R.A. Otunla², Okoronkwo Bertha Francis ${ }^{2}$ and Chukwu Miriam Orji $\mathbf{2}^{2}$

${ }^{* 1}$ National Biotechnology Development Agency of Nigeria

${ }^{21}$ National Biotechnology Development Agency of Nigeria, Bioresources Development Centre (Out Station) UbuluUku, Delta State

${ }^{*}$ Corresponding Author: katstancity@gmail.com
\end{abstract}

Ethnobotany Research \& Applications 22:13 (2021)

\section{Research}

\begin{abstract}
Background: The inadequate programs established to eradicate numerous health problems in Nigeria have led to little improvement in the health status, especially in southern Nigeria. Southern Nigeria has a high prevalence rate of malaria, typhoid, fevers, colds and chills, catarrh, flu, river blindness, respiratory disorders, eye problems and skin infections. The strain caused by the dire need to provide a financial health coverage for the family, a poorly developed health care systems and functional surveillance has led to the exploration of alternative medicine by the indigenes of southern Nigeria.This study aims at documenting information on the common plant resources employed in the ethnomedicinal practices of the indigenous people of the Southern Nigeria, and to explore ways of sensitizing genuine conservation efforts in the face of threat of genetic erosion posed to these resources due to anthropogenic activities.
\end{abstract}

Materials and methods: Onsite ethnomedicinal survey in the study area was carried out between September 2019 and November 2020 to document an indigenous medicinal plant traditional knowledge. Interviews were conducted with the aid of a local language interpreter. Data were obtained using 300 semi-structured questionnaires. Consultations were made on all available information about traditional medicinal plants and ethnomedicinal surveys in Southern Nigeria. Online electronic databases including Google scholar, Research Gate, SciFinder, ScienceDirect and Open Thesis were used to search for relevant literature. Ethnomedicinal data were analyzed using the Relative frequency of citation (RFC), Fidelity level (FL), Relative popularity level (RPL), Use value (UV) and Informant Consensus Factor (ICF).

Results: A total of 236 species belonging to 80 families were reported by this study. Fabaceae was the most represented family having thirty (30) plant species. The three (3) regions had varying frequencies of occurring plants species. South-Western Nigeria represented the region with the highest plant occurrence (47\%) followed by South- 
South (31\%). Leaves (42.32\%) were the most common parts used in the preparation of herbal remedies. Decoctions $(48.89 \%)$ were the most common method of plant preparation used in herbal remedies. Regional distribution and occurrence of ethnomedicinal plant resources of Southern Nigeria is reported here for the first time.

Conclusion: Medicinal plants play crucial role in the treatment of various ailments by the indigenous people in Southern Nigeria. This study highlights the level of species richness as well as biodiversity in the study area. Bioactivity and toxicity by in vitro and in vivo standard tests should be made on herbal drug extracts of the presented species for isolation and possible identification of potentially active compounds

\section{Background}

Ethnomedicinal plants are components of effective source of both traditional and modern medicine. In recent times their potency has been proven and approximately $80 \%$ of the rural population depend on them as a source of health care (Akinyemi et al. 2018 and Abd El-Ghani 2016). Traditional medicine has always been popular among countries of the developing world and its use is becoming acceptable in the industrialized nations (Akinyemi et al. 2000).

As defined by WHO (1978), traditional medicine is the total of all knowledge and practical application, whether explicable or not used in diagnosis, prevention, and elimination of physical, mental or social imbalance, and relying exclusively on practice and experience and observations handed down from generation to generation, whether verbally or in writing.

Since ancient times people across the continents including Africa and most notably West Africa, have relied on plants as sources of remedies for the treatment of many diseases (Abd El-Ghani 2016). According to Hostellmann and Marston (2002), orthodox drugs are expensive in developing countries especially West Africa.

Plant genetic resources of Nigeria are a veritable source of therapeutics and pharmaceuticals though the plants are not adequately documented (Gbile \& Adesina 1986). Ethnomedicinal practice have long been in existence in southern Nigeria but currently undergoing degradation because of pressure from practitioners of modern medical practice and the lack of proper scientific background in its method of administration. The worldwide renewed interest in traditional medicine is due to insufficient availability of orthodox medicine in poor countries, and the sustenance of healthcare has been achieved by these cultural alternatives (Okujagu 2005). Noteworthy is that despite the renewed interests in the use of ethnomedicinal plants, many of the plants may have gone into extinction long before they are documented (Eke 1999). Over-exploitation of wild population of Plant species, anthropogenic activities and lack of conservation programmes are the major problems encountered with sustainable management of these plant resources, especially in the Southern part of Nigeria (Wambebe 1998).

It has been observed that ethnomedicinal practitioners tend to hold in secret the identity of plants used for different ailment for fear of lack of future patronage should the sufferer learn to cure himself. To mystify their trade, cultivation of the plants is not encouraged, thus all the collections are virtually from the wild. With the passing away of most of these practitioners along with their wealth of knowledge, a huge loss is made in the body of knowledge dealing with the plants that heal. Often, the discerning ones try to relate this important information to a few close relatives where any interest is shown. This mode of transmission is, however, grossly inadequate in that it lacks continuity (Obute and Osuji 2002).

Health information system (HIS) is a structured repository of data, information, or knowledge that provides support in health care delivery or to promote health development. Health care provision in Nigeria is a function of the three tiers of Government, Federal, State and Local Governments (Adeyemo 2005, Omoruan et al. 2009, NHIS 1999). The primary health care system in southern Nigeria is managed by Local Government and supported by the southern States Ministry of Health and private medical practitioners (Olanrewaju \& Akanni 2010, Adeyemo 2005). There are some challenges related to the Health Information System (Onwujekwe et al. 2010, Uzochukwu et al. 2015) and this makes the Federal Government unable to take lead roles in directing stakeholders in the health sector and this has resulted to increased levels of fragmentation (Adeyemo 2005).

Population health is determined by environmental, behavioral, genetic, demographic, social, and economic factors. Medical intelligence and surveillance are important components in the health care system and help to control disease outbreak, bio-attack etc. The role of automated based medical intelligence and surveillance systems alongside traditional manual pattern of document retrieval is widespread in Europe and the West. In contrast, the 
Nigerian health care system is still poorly developed, and lacks adequate and functional surveillance systems (Menizibeya 2011). Presently, the strain caused by the dire need to provide a financial health coverage for the family, a poorly developed health care system and functional surveillance has led to the exploration of alternative medicine by the indigenes of southern Nigeria (Olanrewaju \& Akanni 2010).

The inadequate programs designed to address the numerous health problems in Nigeria have led to little improvement in the health status, especially in southern Nigeria (Kajang \& Keswet 2016). From causes such as poor nutrition, poor health facilities, availability of trained medical personnel etc. to mode of living, ways of life and occupational hazards involved in occupations like fishing, wine tapping farming. Environmental factors such as those living in the riverine areas and absence of municipal sewage systems has increased the level of waterborne diseases, and as a result has caused major increase in the health challenges of persons living in these regions.

Southern Nigeria has a high prevalence rate of malaria, as studies have shown that the highest prevalence rates are found in the Niger Delta States, and areas surrounding the confluence of the rivers Niger and Benue. All Nigerians are at risk of malaria and the problem is compounded by the increasing resistance of malaria and the cost of effective drugs (Jimoh et al. 2007, Okonko et al. 2009, Nnadozie 2015, Ezenduka et al. 2017). Other ailments prevalent in the region include typhoid (Ojo et al. 2009, Clark et al. 2010), fevers, colds and chills, catarrh, flu, river blindness (Murray et al. 2013), respiratory disorders, eye problems, worm infection (Gillespie 2018), stomach infections (Bryce, et al. 2005, Ryan 2016) and skin infections.

The need to review ethnomedicinal plants in Southern Nigeria and their various uses cannot be overemphasized for a number of reasons which includes, a growing number of household resort to alternative medicine for health provisions, widespread use of plants in folk medicine, need to conserve traditional medicinal plants and proper documentation of knowledge about them in order to curtail their imminent loss or erosion. This study aims at documenting information on the common plant resources employed in the ethnomedicinal practices of the indigenous people of the Southern Nigeria, and to explore ways of sensitizing genuine conservation efforts in the face of the genetic erosion threat posed to these resources due to anthropogenic activities.

\section{Materials and Methods \\ Demographic Data of Southern Nigeria}

The study was carried out in Southern Nigeria (Fig. 1) which is made up of three (3) regions (geopolitical zones) namely: the south-east region (S.E) comprising of Anambra state, Imo state, Abia state, Enugu state, Ebonyi state, south-south region (S.S) comprising of Delta state, Edo state, Bayelsa state, Rivers state, Cross rivers state and Akwa-ibom state, and south-west region (S.W) comprising of Lagos state, Ondo state, Ogun state, Ekiti state, Oyo state and Osun state (Fig. 1). These regions are characterized by high rainfall and high humidity for most of the year with an average annual rainfall of $250 \mathrm{~cm}$ near the coastal areas and $150 \mathrm{~cm}$ in the northern parts of the region. These regions consisted of different ethnic groups of which six (6) were predominant namely, Yoruba (Y.), Igbo (I.), Edo (E.), Bini (B.), Urhobo (U.), Efik (E.), Ikwerre (IK.), Ibibio (Ib.), Ekpere (Ek.), Kalabari (K.), Ogoni (O.), Oboso-Mbube (O-M.) and ljaw (lj.).

\section{Data collection and ethical procedures}

The informants were briefed on the objectives of the study and informed consent was obtained from each informant. Onsite ethnomedicinal survey in the study area was carried out between September 2019 and November 2020 to document an indigenous medicinal plant traditional knowledge. Interviews were conducted with the aid of a local language interpreter where necessary. Information such as local names, therapeutic use, plant part used, mode of preparation and regional distribution pattern were obtained through the use of semi-structured questionnaires (Huntington 2000). One hundred questionnaires were administered to the informants in each of the three geopolitical zones which make up Southern Nigeria and this summed up to a total of 300 questionnaires administered for the study. Consultations were made on all available information about traditional medicinal plants and ethnomedicinal surveys in Southern Nigeria. A total of 200 male and female informants from all age-groups were randomly selected for the interview, males comprised $48 \%$ and females $52 \%$ of which, 80 were traditional herb sellers, 65 community elders and 55 were herbal practitioners (Tables $1 \& 2$ ). Sixty informants from the total number of informants were interviewed with the aid of a local language interpreter. 


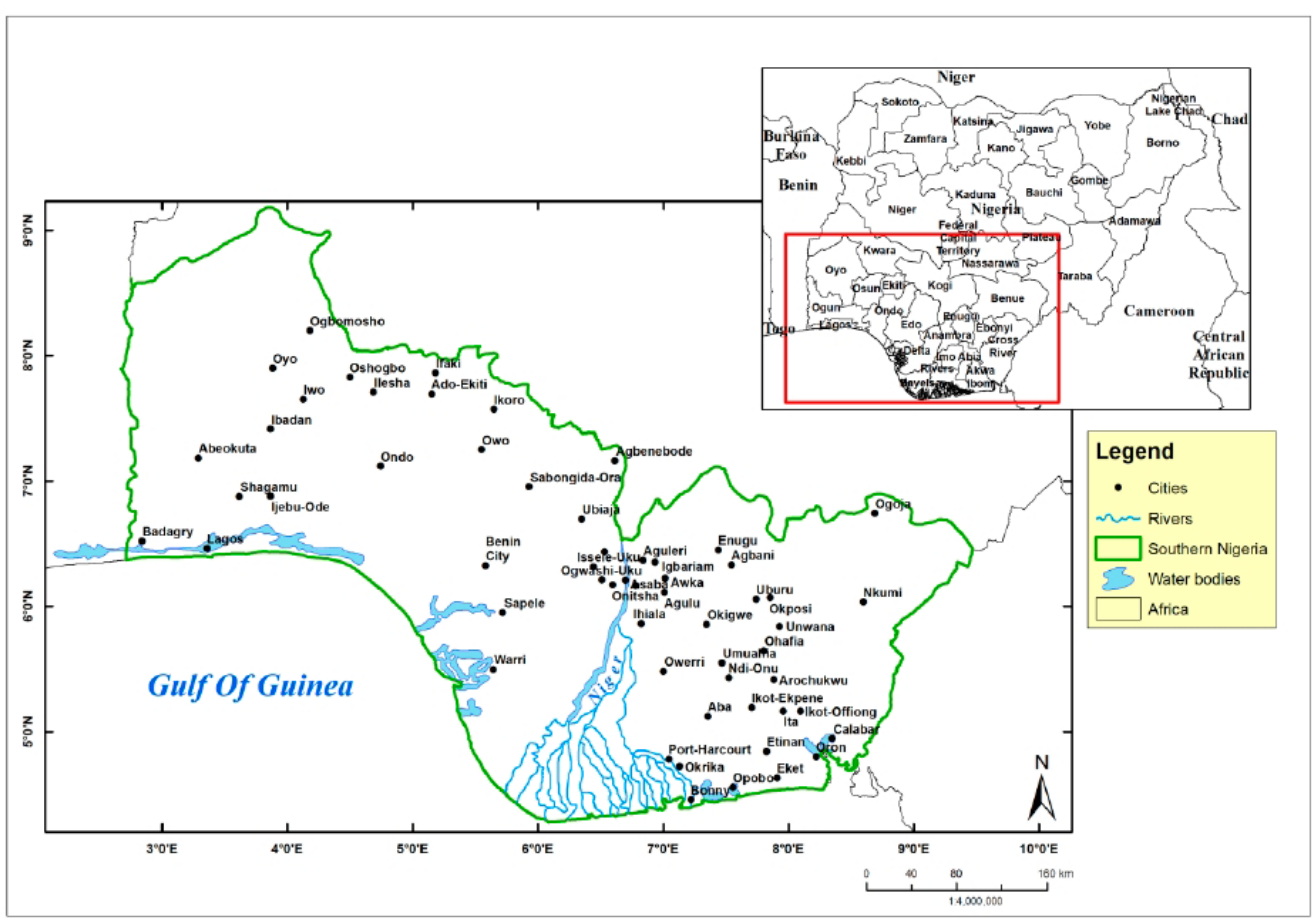

Figure 1. Location of the study area.

Table 1. Demographic data of informant on ethnomedicinal Plant species and their therapeutic application

\begin{tabular}{|l|c|c|c|c|}
\hline Respondents & $\begin{array}{c}\text { Herbal } \\
\text { vendors }\end{array}$ & $\begin{array}{c}\text { Herbal } \\
\text { practitioners }\end{array}$ & $\begin{array}{c}\text { Community } \\
\text { elders }\end{array}$ & $\begin{array}{c}\text { Total } \\
\text { interviewed } \\
\text { persons }\end{array}$ \\
\hline Male $(<40)$ & 10 & 6 & - & 16 \\
\hline Male $(>40)$ & 25 & 28 & 27 & 80 \\
\hline Female (<40) & 12 & 8 & - & 20 \\
\hline Female (>40) & 33 & 13 & 38 & 84 \\
\hline Total interviews & 80 & 55 & 65 & 200 \\
\hline Percentage (\%) men & 44 & 62 & 42 & 48 \\
\hline Percentage (\%) women & 56 & 38 & 58 & 52 \\
\hline
\end{tabular}

Table 2. Data on the trade practice of respondents

\begin{tabular}{|l|l|l|l|l|}
\hline Respondent & $\begin{array}{l}\text { Herbal } \\
\text { sellers }\end{array}$ & $\begin{array}{l}\text { Herbal } \\
\text { practitioners }\end{array}$ & Community elders & $\begin{array}{l}\text { Total } \\
\text { interviewed } \\
\text { persons }\end{array}$ \\
\hline$<40$ & 22 & 14 & - & 36 \\
\hline$>40$ & 58 & 41 & 65 & 164 \\
\hline Total interviews & 80 & 55 & 65 & 200 \\
\hline$\%<40$ & 27 & 25 & - & 18 \\
\hline$>40$ & 73 & 75 & 100 & 82 \\
\hline
\end{tabular}

\section{Authentication and validation of species}

Medicinal plants reported in the survey were collected, identified using Trees of Nigeria (Keay 1989), flora of Nigeria and West Africa (Hutchinson \& Dalziel 1954, 1958, 1968). The identified plants were pressed and deposited at the Bioresources Development Centre, Ubulu-Uku Herbarium (BDU), Delta state. Taxonomic names of plant species were validated from online databases like: The Plant List (http://www.theplantlist.org/), International Legume Database and Information Service (http://www.ildis.org/)and encyclopedia of life (https://eol.org/). Data obtained were collected and tabulated to ascribe botanical names, common names, vernacular names, families of the various plant species as well as their uses. 
Online electronic databases including Google scholar, Research Gate, SciFinder, ScienceDirect, IJPURB and Open Thesis were used to search for relevant literature on previous studies. The key words employed in the electronic search criteria were "Ethnomedicinal surveys", locations of the surveys South-South, South-East and South-West Nigeria. The following key words were used alongside ethnomedicinal practices, health information systems, medical intelligence, and surveillance.

\section{Data analysis}

Data obtained from the study were cleaned prior processing and were analyzed using Statistical Package for Social Science (SPSS) version 23 and Microsoft Office Excel 2016. Socio-demographic data of the respondents were analyzed using a simple descriptive statistical method and reported in a summary of frequency and percentages. On the other hand, ethnomedicinal data were analyzed using the Relative citation frequency (RFC), Fidelity level (FL), Relative popularity level (RPL), Use value (UV) and Informant Consensus Factor (ICF).

\section{Relative frequency of citation (RFC)}

Calculations were made for the local importance of each plant species based on the relative frequency of citation (Tardio et al. 2006). The RFC was calculated as follows: number of who mentioned the use of the species (FC) divided by the total number of respondents $(\mathrm{N})$.

\section{$\mathrm{RFC} 1 / 4 \mathrm{FC} / \mathrm{N}$.}

Informant consensus factor (ICF)

Informant consensus factor was calculated in accordance with Herinch et al. (1998) for each category of ailment to authenticate the level of agreement by the informants on the reported cures for a group of ailments.

$I C F=\left(N_{u r}-N_{t}\right) / N_{u r}-1$

Where Nur = number of use citations in each category

$\mathrm{N}_{\mathrm{t}}=$ number of species used

Fidelity Level

Fidelity Level was calculated in accordance with Alexiades and Sheldon (1996) for the most frequently reported diseases as

$\mathrm{FL}(\%)=\mathrm{N}_{\mathrm{p}} / \mathrm{N}$

Where $N_{p}=$ number of informants that claim a plant use to treat a particular disease $N$ =number of informants that use the plant as medicine in the treatment of any disease

Relative Popularity Level

Relative popularity level was calculated using the formula below in accordance with Ali-Shtayeh et al. (2000).

$\mathrm{RPL}=$ Number of diseases treated by a specific plant species / Total number of informants for any disease

Use value

Use value for each species was calculated in accordance with Savkin et al. (2013) as

Use value $(U V)=\sum u i / N$

Where ui $=$ number of uses recorded for each species $n=$ number of diseases treated by the species

\section{Results}

A total of 236 species were reported by this study and this is shown in Table 3. Study quality inconsistencies were recorded in line with local and common names, completeness of herbal drug recipe, plant parts used, ailments treated and route of herbal drug preparation. Two hundred informants provided information on herbal remedies used in 22 ethnomedicinal therapeutic applications (Table 5). The use of single plant species (5\%) as well as multiple uses of plant species (95\%) in the treatment of various ailments is reported. This study revealed important information gaps that should be addressed as well as the need for standardization of ethnomedicinal practices and studies in Southern Nigeria. 


\section{Frequency of family occurrence}

A total of eighty (80) families were reported in this study. The results obtained from this study revealed, the family of Fabaceae had the highest frequency of occurrence having thirty (30) plant species, followed by Asteraceae with fourteen (14) species, Euphorbiaceae having thirteen (13) species, Rutaceae having nine (9) species, Curcubitaceae (8), Annonaceae, Combretaceae, Rubiaceae and Verbenaceae having (6) species each, as well as Apocynaceae, Lamiaceae, Meliaceae and Sterculiaceae having five (5) species each (Table 3, Fig. 2 ).

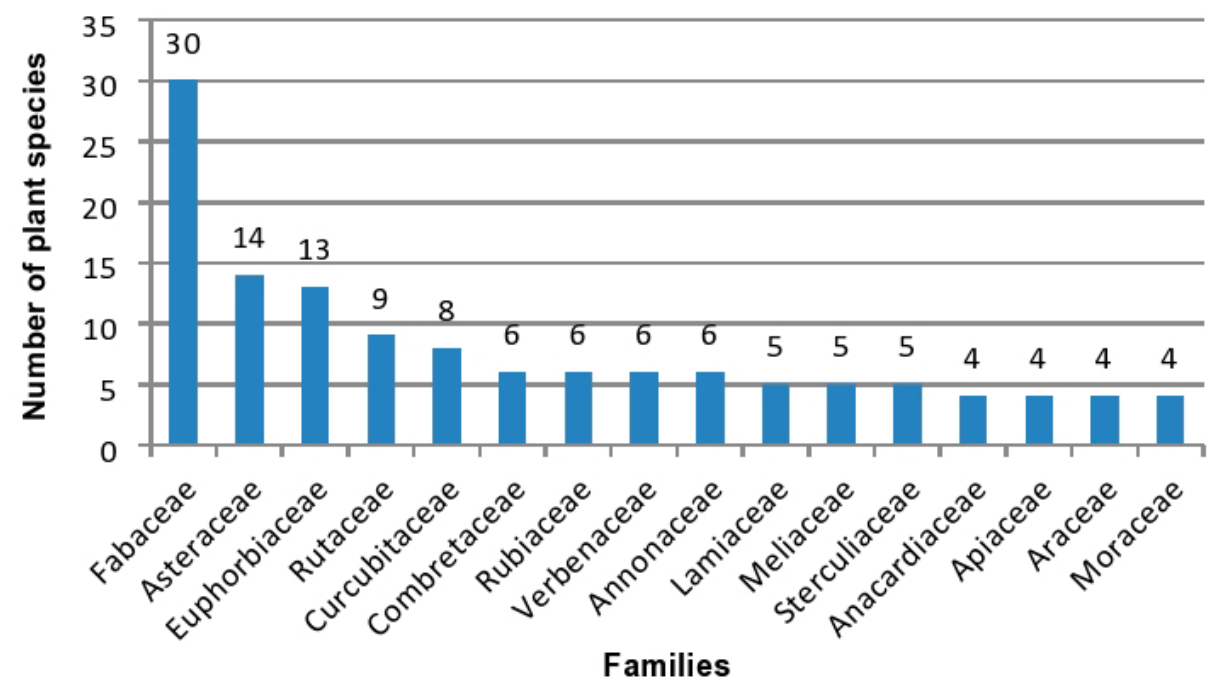

Figure 2. Top sixteen representative families of medicinal plants in Southern Nigeria.

\section{Frequency of plants regional occurrence}

In the study area (Southern Nigeria), the three (3) regions had varying frequencies of occurring plants species. South-Western Nigeria represented the region with the highest plant occurrence (47\%) followed by South-South (31\%) and South-East (22\%) (Fig. 3).

\section{Frequency of plants parts used}

In the study area, the leaves (42.32\%) were the most common parts used in the preparation of herbal remedies followed by the stem/stembark (20.63\%), roots (14\%), seeds (6.87\%), fruits (7.93\%), whole plant (3.9\%), sap (1.32\%), rhizome $(0.52 \%)$, corms, flowers, oils, pulp, and gum exudates each having $0.26 \%$ (Fig. 4 ).

\section{Frequency of plant preparation}

In the study area (Fig. 5), decoction (48.89\%) was the most common method of plant preparation used in herbal remedies followed by infusions (15.29\%), poultices (11.33\%), juice extracts (8.78\%), mastication (5\%), tinctures (4.53\%), macerations (2.54\%), baths and remedies (1.98\%), aromatherapy and powdered each having $1.41 \%$, compresses and syrups each having (0.84\%).

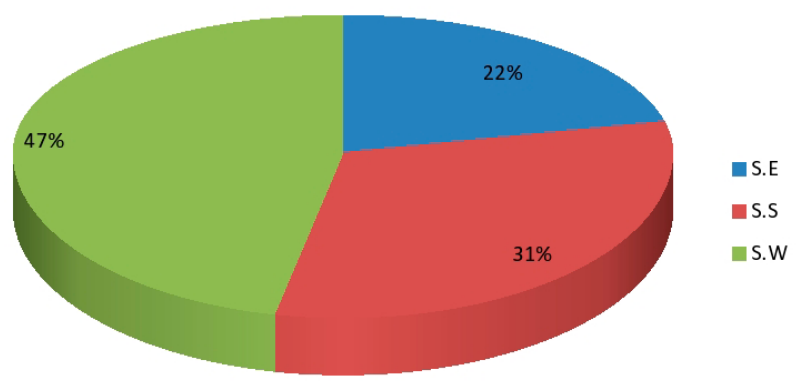

Figure 3. Frequency of plants regional occurrence. 
Table 3. Ethnomedicinal Plants in Southern Nigeria

\begin{tabular}{|c|c|c|c|c|c|c|c|c|c|c|c|c|}
\hline & Family & Local name & $\begin{array}{l}\text { Common } \\
\text { name }\end{array}$ & Therapeutic usage & Parts used & $\begin{array}{l}\text { Method of } \\
\text { preparation }\end{array}$ & 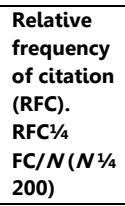 & $\begin{array}{l}\text { Fidelity } \\
\text { level }\end{array}$ & $\begin{array}{l}\text { Relative } \\
\text { popularity } \\
\text { level }\end{array}$ & $\begin{array}{l}\text { Use } \\
\text { valu } \\
\text { e }\end{array}$ & $\begin{array}{l}\text { Regional } \\
\text { distributio } \\
\text { n pattern }\end{array}$ & References \\
\hline $\begin{array}{l}\text { Abrus precatorius } \mathrm{L} . \\
\text { BDU } 106\end{array}$ & Fabaceae & $\begin{array}{l}\text { Anyannun (K.), Oju- } \\
\text { ologbo (Y.) }\end{array}$ & $\begin{array}{l}\text { Crab's eye, } \\
\text { Bead tree }\end{array}$ & $\begin{array}{l}\text { Malaria, eye infection, } \\
\text { skin disease, cough }\end{array}$ & Leaves & Decoction & 0.26 & 73 & 0.2 & 0.07 & s.s, s.w & lyama \& Idu 2015 \\
\hline $\begin{array}{l}\text { Acacia nilotica (L.) Wild ex Del } \\
\text { BDU } 133\end{array}$ & Fabaceae & Boonii (H.) & $\begin{array}{l}\text { Acacia, } \\
\text { Egyptian } \\
\text { mimosa }\end{array}$ & Malaria & Seeds & Decoction & 0.19 & 55 & 0.05 & 0.02 & s.e, s.s, s.w, & lyama \& Idu 2015 \\
\hline $\begin{array}{l}\text { Acacia senegal (L.) Wild } \\
\text { BDU } 51\end{array}$ & Fabaceae & Dakwara (H.) & $\begin{array}{l}\text { Acacia, } \\
\text { Gum } \\
\text { Arabic }\end{array}$ & Malaria & Stembark & Decoction & 0.1 & 60 & 0.05 & 0.05 & s.e, s.s, s.W & lyama \& Idu 2015 \\
\hline $\begin{array}{l}\text { Acalypha fimbriata (Schum \& } \\
\text { Thonn) Forsk } \\
\text { BDU } 91\end{array}$ & Euphorbiaceae & Abalebaji (K.) & $\begin{array}{l}\text { Copper } \\
\text { leaves }\end{array}$ & $\begin{array}{l}\text { Heart failure, cough } \\
\text { skin disease, boils }\end{array}$ & $\begin{array}{l}\text { Leaves, } \\
\text { twig }\end{array}$ & Juice, decoction & 0.11 & 68 & 0.2 & 0.18 & s.w & $\begin{array}{l}\text { Ajibesin et al. } \\
2008 \text { \& } 2012\end{array}$ \\
\hline $\begin{array}{l}\text { Acanthospermum hispidum D.C } \\
\text { BDU } 47\end{array}$ & Asteraceae & $\begin{array}{l}\text { Dagunro (Y.), Gorogoro } \\
\text { (U.) }\end{array}$ & Starburr & Malaria & Leaves & Decoction & 0.09 & 50 & 0.05 & 0.05 & s.s, s.w & $\begin{array}{l}\text { lyama \& Idu } \\
2015\end{array}$ \\
\hline $\begin{array}{l}\text { Acanthus montanus (Nees) T. } \\
\text { Anders } \\
\text { BDU } 10\end{array}$ & Acanthaceae & $\begin{array}{l}\text { Inyinyiogwu (I.), Oga } \\
\text { (IK.) }\end{array}$ & False thistle & $\begin{array}{l}\text { Chesty coughs, boils, } \\
\text { Chest pain, heart } \\
\text { failure, gonorrhea, } \\
\text { wounds }\end{array}$ & $\begin{array}{l}\text { Leaves, } \\
\text { roots }\end{array}$ & $\begin{array}{l}\text { Decoction, } \\
\text { tincture, poultice }\end{array}$ & 0.22 & 70 & 0.3 & 0.13 & s.e, s.s, s.w & $\begin{array}{l}\text { Alade et al. 2018, } \\
\text { Akwaji et al. } 2017\end{array}$ \\
\hline $\begin{array}{l}\text { Achyranthes aspera L. } \\
\text { BDU } 12\end{array}$ & Amaranthaceae & Aboro, Abora (Y.) & $\begin{array}{l}\begin{array}{l}\text { Prickly } \\
\text { chaff flower }\end{array} \\
\end{array}$ & Malaria & $\begin{array}{l}\text { Leaves, } \\
\text { roots }\end{array}$ & Decoction & 0.06 & 75 & 0.05 & 0.08 & s.w & lyama \& Idu 2015 \\
\hline $\begin{array}{l}\text { Adansonia digitata L. } \\
\text { BDU } 93\end{array}$ & Bombacaceae & Ose (Y.) & $\begin{array}{l}\text { Baobab } \\
\text { tree }\end{array}$ & $\begin{array}{l}\text { Arthritis, malaria, } \\
\text { rheumatism, } \\
\text { toothache, asthma, } \\
\text { dysentry }\end{array}$ & $\begin{array}{l}\text { Leaves, } \\
\text { stembark, } \\
\text { seeds, latex, } \\
\text { pulp }\end{array}$ & $\begin{array}{l}\text { Decoction, } \\
\text { tincture, poultice }\end{array}$ & 0.19 & 71 & 0.3 & 0.15 & s.s, s.w & $\begin{array}{l}\text { Akwaji et al. } \\
\text { 2017, Iyama \& } \\
\text { Idu2015, Lawal et } \\
\text { al. } 2010\end{array}$ \\
\hline $\begin{array}{l}\text { Adenopus breviflorus Benth. } \\
\text { BDU } 160\end{array}$ & Cucurbitaceae & Ukuro (EK.) & & Convulsion, laxative & $\begin{array}{l}\text { Leaves, } \\
\text { fruits }\end{array}$ & $\begin{array}{l}\text { Decoction, } \\
\text { infusion }\end{array}$ & 0.09 & 77 & 0.1 & 0.11 & s.s, s.e & $\begin{array}{l}\text { Ajibesin et al. } \\
2008 \& 2012\end{array}$ \\
\hline $\begin{array}{l}\text { Adenostemma mauritianum DC. } \\
\text { BDU } 03\end{array}$ & Asteraceae & Oforu (IK.) & & Malaria, measles & Leaves & Infusion & 0.05 & 60 & 0.1 & 0.2 & s.s & $\begin{array}{l}\text { Ajibesin et al. } \\
2008 \& 2012\end{array}$ \\
\hline $\begin{array}{l}\text { Afromomum melegueta roscoe } \\
\text { K. Schum } \\
\text { BDU } 31\end{array}$ & Zingiberaceae & $\begin{array}{l}\text { Ose-oji, (I.), Erhie (U.), } \\
\text { Atare (Y.) }\end{array}$ & $\begin{array}{l}\text { Alligator } \\
\text { pepper, } \\
\text { grains of } \\
\text { paradise, } \\
\text { guinea } \\
\text { grains }\end{array}$ & $\begin{array}{l}\text { Diabetes, female } \\
\text { infertility, vermifuge, } \\
\text { smallpox, chickenpox, } \\
\text { catarrh, chest } \\
\text { congestion, malaria, } \\
\text { hypertension, } \\
\text { measles, sore throat, } \\
\text { cholera, aphrodisiac }\end{array}$ & $\begin{array}{l}\text { Leaves, } \\
\text { seeds, } \\
\text { stembark }\end{array}$ & $\begin{array}{l}\text { Decoction, } \\
\text { aromatherapy, } \\
\text { mastication, } \\
\text { macerations in } \\
\text { Mimordica } \\
\text { charantha and } \\
\text { Sorghum } \\
\text { arundinaceum }\end{array}$ & 0.23 & 78 & 0.7 & 0.3 & s.e, s.s, s.w & $\begin{array}{l}\text { lyama \& Idu } \\
2015, \text { Alade et al. } \\
2018\end{array}$ \\
\hline $\begin{array}{l}\text { Ageratum conyzoides L. } \\
\text { BDU } 184\end{array}$ & Asteraceae & $\begin{array}{l}\text { Ulanjula, uratanjele (I.), } \\
\text { Ogba-Okukpo (K.), } \\
\text { Ako-yunyun (Y.) }\end{array}$ & Goat weed & $\begin{array}{l}\text { Wounds, eye } \\
\text { infections, malaria, } \\
\text { skin diseases }\end{array}$ & Leaves & $\begin{array}{l}\text { Infusion, juice } \\
\text { extract }\end{array}$ & 0.19 & 84 & 0.2 & 0.1 & s.e, s.s, s.W & Iyama \& Idu 2015 \\
\hline
\end{tabular}


Ethnobotany Research and Applications

\begin{tabular}{|c|c|c|c|c|c|c|c|c|c|c|c|c|}
\hline $\begin{array}{l}\text { Alchornea cordifolia (Schum } \\
\text { \&Thonn.) Müll.-Arg. } \\
\text { BDU } 53\end{array}$ & Euphorbiaceae & $\begin{array}{l}\text { Ubebe (I.), Epai (IK.), } \\
\text { Epa, Esin (Y.) }\end{array}$ & $\begin{array}{l}\text { Christmas } \\
\text { bush }\end{array}$ & $\begin{array}{l}\text { Eye problem, as a } \\
\text { detox bitter, wounds, } \\
\text { Toothache, } \\
\text { hemorrhoid, } \\
\text { ringworm, } \\
\text { rheumatism, } \\
\text { gonorrhea, urethral } \\
\text { disease, dysentry }\end{array}$ & $\begin{array}{l}\text { Leaves, } \\
\text { stembark, } \\
\text { root } \\
\text { epidermis }\end{array}$ & $\begin{array}{l}\text { Decoction, juice, } \\
\text { mastication, } \\
\text { maceration }\end{array}$ & 0.23 & 76 & 0.5 & 0.21 & s.e, s.s, s.w & $\begin{array}{l}\text { Ariwaodo et al. } \\
\text { 2012, Akwaji et } \\
\text { al. } 2017\end{array}$ \\
\hline $\begin{array}{l}\text { Allanblackia floribunda Oliv. } \\
\text { BDU } 57\end{array}$ & Clusiaceae & $\begin{array}{l}\text { Egba (I.), Orogboerin } \\
\text { (Y.) }\end{array}$ & Fallow tree & Malaria, tooth ache & Leaves & Decoction & 0.07 & 85 & 0.1 & 0.14 & s.e, s.w & $\begin{array}{l}\text { Akwaji et al. } \\
\text { 2017, lyama \& } \\
\text { Idu } 2015\end{array}$ \\
\hline $\begin{array}{l}\text { Allium cepa L. } \\
\text { BDU } 90\end{array}$ & Liliaceae & Alubosa (Y.), Uta (E.) & Onions & $\begin{array}{l}\text { Malaria, convulsions, } \\
\text { faintings }\end{array}$ & $\begin{array}{l}\text { Leaves, } \\
\text { whole bulb, } \\
\text { stembark }\end{array}$ & $\begin{array}{l}\text { Decoction, } \\
\text { topical } \\
\text { application }\end{array}$ & 0.13 & 50 & 0.15 & 0.11 & s.s, s.w & Iyama\&Idu 2015 \\
\hline $\begin{array}{l}\text { Allium sativum L. } \\
\text { BDU } 35\end{array}$ & Amaryllidaceae & $\begin{array}{l}\text { Ayuu (Y.), Ayo (U.), Uta } \\
\text { (E.) }\end{array}$ & $\begin{array}{l}\text { Garlic, } \\
\text { Haemorrha } \\
\text { ge Plant }\end{array}$ & $\begin{array}{l}\text { Highblood pressure, } \\
\text { malaria, fever, } \\
\text { indigestion and as a } \\
\text { tonic }\end{array}$ & $\begin{array}{l}\text { Leaves, } \\
\text { stembark }\end{array}$ & $\begin{array}{l}\text { Decoction, } \\
\text { mastication }\end{array}$ & 0.17 & 88 & 0.25 & 0.14 & s.s, s.w & Alade et al. 2018 \\
\hline $\begin{array}{l}\text { Aloe vera (L.) Burm. } f \\
\text { BDU } 16\end{array}$ & Liliaceae & & $\begin{array}{l}\text { Barbados } \\
\text { Aloe }\end{array}$ & $\begin{array}{l}\text { Malaria, hair growth, } \\
\text { wounds,skin } \\
\text { infections, } \\
\text { dysmenorrhea }\end{array}$ & Leaves & Decoction & 0.07 & 71 & 0.3 & 0.29 & s.e, s.s, s.w & $\begin{array}{l}\text { Alade\&Ajibesin } \\
\text { 2017, Iyama \& } \\
\text { Idu } 2015\end{array}$ \\
\hline $\begin{array}{l}\text { Alstonia boonei DeWild } \\
\text { BDU } 44\end{array}$ & Apocynaceae & $\begin{array}{l}\text { Egbu (I.), Ulodiri (EK.), } \\
\text { Ahun (Y.) }\end{array}$ & & $\begin{array}{l}\text { Tonic, malaria, } \\
\text { asthma, cough, } \\
\text { rheumatism, } \\
\text { gonorrhoea, } \\
\text { vermifuge, bladder } \\
\text { disease }\end{array}$ & $\begin{array}{l}\text { Leaves, } \\
\text { stembark, } \\
\text { roots }\end{array}$ & $\begin{array}{l}\text { Decoction, } \\
\text { topical } \\
\text { applications, } \\
\text { poultice }\end{array}$ & 0.22 & 90 & 0.4 & 0.18 & s.e, s.w & $\begin{array}{l}\text { Ariwaodoet al. } \\
\text { 2012, Akwaji et } \\
\text { al. } 2017\end{array}$ \\
\hline $\begin{array}{l}\text { Anacardium occidentale L. BDU } \\
88\end{array}$ & Anacardiaceae & $\begin{array}{l}\text { Kanshu(I.), Kasu (Y.), } \\
\text { Kasiu (IK.) }\end{array}$ & Cashew & $\begin{array}{l}\text { Fever, malaria, tooth } \\
\text { aches, diarrhea, } \\
\text { kidney problem, } \\
\text { whooping cough }\end{array}$ & $\begin{array}{l}\text { Leaves, } \\
\text { stembark }\end{array}$ & $\begin{array}{l}\text { Decoction, } \\
\text { poultice, tincture }\end{array}$ & 0.19 & 84 & 0.4 & 0.18 & s.e, s.s, s.w & $\begin{array}{l}\text { Akwaji et al.2017, } \\
\text { lyama \& Idu } \\
2015 \text {, Lawal et al. } \\
2010\end{array}$ \\
\hline $\begin{array}{l}\text { Ananas comosus (L). Merr. } \\
\text { BDU } 162\end{array}$ & Bromeliaceae & $\begin{array}{l}\text { Ope-Oyibo (U.), Ediebo } \\
\text { (EK.) }\end{array}$ & Pineapple & $\begin{array}{l}\text { Fever, malaria, } \\
\text { hepatitis, typhoid, } \\
\text { menstrual disorder, } \\
\text { waist pain, purgative, } \\
\text { expectorant, } \\
\text { emmenagogue }\end{array}$ & Fruits & Decoction & 0.15 & 80 & 0.5 & 0.3 & 5.5 & $\begin{array}{l}\text { Ariwaodo et al. } \\
2012 \text {, lyama \& Idu } \\
2015\end{array}$ \\
\hline $\begin{array}{l}\text { Anethum graveolens L. } \\
\text { BDU } 111\end{array}$ & Apiaceae & Udumie (K.) & Dill weed & $\begin{array}{l}\text { Laxative, improved } \\
\text { lactation }\end{array}$ & Seeds & $\begin{array}{l}\text { Decoction, } \\
\text { infusion with } \\
\text { honey }\end{array}$ & 0.07 & 85 & 0.1 & 0.14 & s.s & $\begin{array}{l}\text { Ajibesin et al. } \\
2008 \& 2012\end{array}$ \\
\hline $\begin{array}{l}\text { Anogeissus leiocarpus (D.C). } \\
\text { Guill. \& Perr } \\
\text { BDU } 64\end{array}$ & Combretaceae & Egbo-anyin (Y.) & Axle wood & Malaria & Roots & Decoction & 0.05 & 60 & 0.05 & 0.1 & s.w & Iyama \& Idu 2015 \\
\hline $\begin{array}{l}\text { Annona muricata L. } \\
\text { BDU120 }\end{array}$ & Annonaceae & Nangi (K.), Abo (Y.) & Sour sop & $\begin{array}{l}\text { Malaria, diarrhea, } \\
\text { dysentery, debility, } \\
\text { hypertension, heart } \\
\text { failure, yellow fever }\end{array}$ & Leaves & Decoction & 0.11 & 86 & 0.4 & 0.32 & s.s, s.w & Lawal et al. 2010 \\
\hline $\begin{array}{l}\text { Anthocleista djalonensis } \\
\text { A. Chev } \\
\text { BDU } 148\end{array}$ & Loganiaceae & Sapo (Y.) & $\begin{array}{l}\text { Cabbage } \\
\text { tree }\end{array}$ & $\begin{array}{l}\text { Malaria, anti-diuretic, } \\
\text { purgative, jaundice }\end{array}$ & Stembark & Decoction & 0.22 & 90 & 0.2 & 0.09 & s.w & $\begin{array}{l}\text { lyama \& Idu } \\
\text { 2015,Lawal et al. } \\
2010\end{array}$ \\
\hline
\end{tabular}


Ethnobotany Research and Applications

\begin{tabular}{|c|c|c|c|c|c|c|c|c|c|c|c|c|}
\hline $\begin{array}{l}\text { Anthonotha macrophylla P. } \\
\text { Beauv } \\
\text { BDU } 79\end{array}$ & Fabaceae & $\begin{array}{l}\text { Ububa-ikpa (I.), Abata } \\
\text { (Y.) }\end{array}$ & & $\begin{array}{l}\text { Veneral diseases, } \\
\text { vermifuge, intestinal } \\
\text { discomfort, analgesic, } \\
\text { skin infections, } \\
\text { venomous stings, } \\
\text { bites }\end{array}$ & $\begin{array}{l}\text { Leaves, } \\
\text { stembark, } \\
\text { roots, gum } \\
\text { exudates }\end{array}$ & $\begin{array}{l}\text { Decoction, } \\
\text { infusions, } \\
\text { poultices }\end{array}$ & 0.06 & 75 & 0.4 & 0.58 & s.e, s.w & $\begin{array}{l}\text { Akwaji et al. } \\
\text { 2017, Lawal et al. } \\
2010\end{array}$ \\
\hline $\begin{array}{l}\text { Artemisia dracunculus L. } \\
\text { BDU } 25\end{array}$ & Asteraceae & Nshegbuawom(O-M.) & Tarragon & $\begin{array}{l}\text { Malaria, typhoid, } \\
\text { Diabetes, skin } \\
\text { disease, cuts, arthritis, } \\
\text { laxative, vermifuge }\end{array}$ & Leaves & $\begin{array}{l}\text { Decoction, } \\
\text { infusion }\end{array}$ & 0.09 & 50 & 0.4 & 0.44 & s.s & $\begin{array}{l}\text { Ajibesin et al. } \\
2008 \& 2012\end{array}$ \\
\hline $\begin{array}{l}\text { Aspilia africana (Pers.) C.D } \\
\text { Adams } \\
\text { BDU } 66\end{array}$ & Asteraceae & $\begin{array}{l}\text { Oranjila (IK.), Ifakop } \\
\text { (O-M.) }\end{array}$ & $\begin{array}{l}\text { Haemorrha } \\
\text { ge plant }\end{array}$ & $\begin{array}{l}\text { Wound clotting and } \\
\text { healing, stomach } \\
\text { disorders }\end{array}$ & Leaves & Juice extract & 0.15 & 90 & 0.15 & 0.1 & s.s & $\begin{array}{l}\text { Alade et al. } \\
\text { 2018,Ariwaodo et } \\
\text { al. } 2012\end{array}$ \\
\hline $\begin{array}{l}\text { Azadirachta indica Juss. } \\
\text { BDU141 }\end{array}$ & Meliaceae & $\begin{array}{l}\text { Dongoyaro, Eke-oyibo } \\
\text { (Y.) }\end{array}$ & Neem & $\begin{array}{l}\text { Malaria, indigestion, } \\
\text { vermifuge }\end{array}$ & $\begin{array}{l}\text { Leaves, } \\
\text { twig }\end{array}$ & $\begin{array}{l}\text { Decoction, } \\
\text { infusion }\end{array}$ & 0.26 & 90 & 0.15 & 0.05 & s.w & $\begin{array}{l}\text { lyama \& Idu } \\
2015, \text { Lawal et al. } \\
2010\end{array}$ \\
\hline $\begin{array}{l}\text { Bambusa vulgaris } \mathrm{L} \text {. } \\
\text { BDU } 123\end{array}$ & Poaceae & Abaji- Okporo (I.) & Bamboo & $\begin{array}{l}\text { Gonorrhea, } \\
\text { hemorrhoids, } \\
\text { vermifuge, respiratory } \\
\text { disease }\end{array}$ & $\begin{array}{l}\text { Young } \\
\text { shoots, } \\
\text { leaves }\end{array}$ & $\begin{array}{l}\text { Decoction, } \\
\text { tincture, poultice }\end{array}$ & 0.09 & 50 & 0.2 & 0.22 & s.e & $\begin{array}{l}\text { Ariwaodo et } \\
\text { al.2012, Akwaji et } \\
\text { al. } 2017\end{array}$ \\
\hline Baphia nitida Lodd. BDU 95 & Fabaceae & $\begin{array}{l}\text { Abode (EK.), Ibali (I.), } \\
\text { Osun (Y.), }\end{array}$ & $\begin{array}{l}\text { African } \\
\text { sandal } \\
\text { wood, cam } \\
\text { wood }\end{array}$ & Cough, boil & $\begin{array}{l}\text { Leaves, } \\
\text { twig, } \\
\text { stembark, } \\
\text { roots }\end{array}$ & $\begin{array}{l}\text { Mastication, } \\
\text { poultice }\end{array}$ & 0.13 & 92 & 0.1 & 0.07 & s.e, s.w & $\begin{array}{l}\text { Alade et al. 2018, } \\
\text { Ariwaodo et al. } \\
\text { 2012, Ajibesin et } \\
\text { al. } 2008 \& 2012 \text {. }\end{array}$ \\
\hline $\begin{array}{l}\text { Baphia pubescence Hook.F } \\
\text { BDU } 45\end{array}$ & Fabaceae & $\begin{array}{l}\text { Awewi, Urohun, } \\
\text { Maajigii (Y.) }\end{array}$ & $\begin{array}{l}\text { Benin - } \\
\text { camwood }\end{array}$ & $\begin{array}{l}\text { Fever, sores, wounds, } \\
\text { ringworm, sterility, } \\
\text { dysmenorrhoea, } \\
\text { sterility, diarrhea, pain }\end{array}$ & $\begin{array}{l}\text { Leaves, } \\
\text { stembark, } \\
\text { roots }\end{array}$ & $\begin{array}{l}\text { Decoction, } \\
\text { infusion }\end{array}$ & 0.03 & 50 & 0.5 & 1.0 & s.w & Lawal et al. 2010 \\
\hline $\begin{array}{l}\text { Basella alba L. } \\
\text { BDU } 33\end{array}$ & Basellaceae & $\begin{array}{l}\text { Amunututu (Y.), } \\
\text { Gbologi (IK.) }\end{array}$ & $\begin{array}{l}\text { Ceylon } \\
\text { spinach }\end{array}$ & $\begin{array}{l}\text { Laxative, boils, hot } \\
\text { flushes }\end{array}$ & $\begin{array}{l}\text { Whole } \\
\text { plant }\end{array}$ & Infusion & 0.07 & 71 & 0.15 & 0.21 & s.s, s.w & $\begin{array}{l}\text { Ajibesin et al. } \\
2008 \text { \& } 2012\end{array}$ \\
\hline $\begin{array}{l}\text { Bixa orellana L. } \\
\text { BDU } 12\end{array}$ & Bixaceae & Ufie, Uhie (I.), Aje (Y.) & Annatto & $\begin{array}{l}\text { Diabetes, laxatives, } \\
\text { dysentery, diarrhoea, } \\
\text { malaria, fevers, } \\
\text { scrapes, burns }\end{array}$ & $\begin{array}{l}\text { Leaves, } \\
\text { fruits, seeds }\end{array}$ & Decoction & 0.06 & 75 & 0.4 & 0.66 & s.e, s.w & Lawal et al. 2010 \\
\hline $\begin{array}{l}\text { Brachystergiaeurycoma Harms. } \\
\text { BDU } 148\end{array}$ & Fabaceae & $\begin{array}{l}\text { Ako, Akolodu (Y.), } \\
\text { Okweri (E.), Apaupan } \\
\text { (IJ.), Odukpa (IB.) }\end{array}$ & & Bone strengthening & Seeds & Powdered & 0.17 & 88 & 0.05 & 0.02 & s.e, s.s, s.w & Lawal et al. 2010 \\
\hline $\begin{array}{l}\text { Brassica nigra (L.) } \\
\text { W.D.J. Koch } \\
\text { BDU } 38\end{array}$ & Brassicaceae & Ogwunje (K.) & $\begin{array}{l}\text { Black } \\
\text { mustard }\end{array}$ & $\begin{array}{l}\text { Hypertension, } \\
\text { rheumatism, } \\
\text { headaches }\end{array}$ & $\begin{array}{l}\text { Leaves, } \\
\text { seeds }\end{array}$ & $\begin{array}{l}\text { Infusion, juice } \\
\text { mixed with onion } \\
\text { juice, poultice }\end{array}$ & 0.11 & 86 & 0.15 & 0.13 & s.s & $\begin{array}{l}\text { Ajibesin et al. } \\
2008 \& 2012\end{array}$ \\
\hline $\begin{array}{l}\text { Brassica oleracea L. } \\
\text { BDU34 }\end{array}$ & Brassicaceae & Ogbeagu (K.) & $\begin{array}{l}\text { Wild } \\
\text { cabbage }\end{array}$ & $\begin{array}{l}\text { Stomach ulcer, } \\
\text { diabetes, wound }\end{array}$ & Leaves & Juice, poultice & 0.06 & 75 & 0.15 & 0.03 & s.s & $\begin{array}{l}\text { Ajibesin et al. } \\
2008 \text { \& } 2012\end{array}$ \\
\hline $\begin{array}{l}\text { Caesalpinia bonduc (L.) Roxb } \\
\text { BDU } 15\end{array}$ & Fabaceae & Ayoo (Y.) & & $\begin{array}{l}\text { Fungal infections, } \\
\text { malaria }\end{array}$ & $\begin{array}{l}\text { Leaves, } \\
\text { young } \\
\text { twigs }\end{array}$ & Decoction & 0.03 & 50 & 0.1 & 0.33 & s.w & Lawal et al. 2010 \\
\hline $\begin{array}{l}\text { Caladium bicolor (Aiton) Vent. } \\
\text { BDU } 20\end{array}$ & Araceae & Honya (EK.) & $\begin{array}{l}\text { Heart of } \\
\text { Jesus }\end{array}$ & Skin diseases, wound & Corm & Poultice & 0.06 & 50 & 0.1 & 0.16 & s.s & $\begin{array}{l}\text { Ajibesin et al. } \\
2008 \& 2012\end{array}$ \\
\hline $\begin{array}{l}\text { Calendula officinalis L. } \\
\text { BDU28 }\end{array}$ & Asteraceae & Ukpuda (IK.) & $\begin{array}{l}\text { Pot } \\
\text { marigold }\end{array}$ & $\begin{array}{l}\text { Urinary tract } \\
\text { infection, } \\
\text { Inflammation }\end{array}$ & Leaves & $\begin{array}{l}\text { Decoction, } \\
\text { poultice }\end{array}$ & 0.07 & 85 & 0.1 & 0.14 & 5.5 & $\begin{array}{l}\text { Ajibesin et al. } \\
2008 \& 2012\end{array}$ \\
\hline $\begin{array}{l}\text { Canavalia ensiformis (L.) DC. } \\
\text { BDU } 22\end{array}$ & Fabaceae & Pokondo (Y.) & Jack bean & Antibiotic, antiseptic & Seeds & & 0.06 & 50 & 0.1 & 0.16 & s.w & Lawal et al. 2010 \\
\hline $\begin{array}{l}\text { Canna indical. } \\
\text { BDU } 37\end{array}$ & Cannaceae & Ido $(Y)$. & Indian shot & $\begin{array}{l}\text { Local birth control, } \\
\text { Malaria }\end{array}$ & Leaves & Decoction & 0.03 & 50 & 0.1 & 0.33 & s.w & Lawal et al. 2010 \\
\hline
\end{tabular}


Ethnobotany Research and Applications

\begin{tabular}{|c|c|c|c|c|c|c|c|c|c|c|c|c|}
\hline $\begin{array}{l}\text { Cannabis sativa L. } \\
\text { BDU } 169\end{array}$ & Cannabinaceae & Igbo & $\begin{array}{l}\text { Indian } \\
\text { hemp plant }\end{array}$ & $\begin{array}{l}\text { Pain, stunted hair } \\
\text { growth }\end{array}$ & $\begin{array}{l}\text { Leaves, } \\
\text { stem }\end{array}$ & $\begin{array}{l}\text { Aromatherapy, } \\
\text { poultice }\end{array}$ & 0.15 & 90 & 0.1 & 0.06 & s.S, s.W & $\begin{array}{l}\text { Alade\&Ajibesin } \\
2017\end{array}$ \\
\hline $\begin{array}{l}\text { Carica papaya L. } \\
\text { BDU } 146\end{array}$ & Caricaceae & $\begin{array}{l}\text { Okpurukwa (I.), Eto- } \\
\text { Oyibo (U.) }\end{array}$ & $\begin{array}{l}\text { Papaya, } \\
\text { papaw }\end{array}$ & $\begin{array}{l}\text { Kidney detox, malaria, } \\
\text { fever, diabetes, } \\
\text { cancer, eczema, after- } \\
\text { shave bumps, waist } \\
\text { pain, syphilis, } \\
\text { nematode } \\
\text { infestations }\end{array}$ & $\begin{array}{l}\text { Leaves, fruit } \\
\text { (ripe and } \\
\text { unripe), } \\
\text { seeds }\end{array}$ & $\begin{array}{l}\text { Decoction, } \\
\text { infusion, } \\
\text { macerations in } \\
\text { Ocimum } \\
\text { gratissimum, } \\
\text { Garcinia kola } \\
\text { seed }\end{array}$ & 0.23 & 76 & 0.6 & 0.24 & s.e, s.s & $\begin{array}{l}\text { Alade\&Ajibesin } \\
2017\end{array}$ \\
\hline $\begin{array}{l}\text { Carpolobia lutea G. Don } \\
\text { BDU } 28\end{array}$ & Polygonaceae & $\begin{array}{l}\text { Agba, Angalagala (I.), } \\
\text { Osunsun (Y.), Ikpafum } \\
\text { (IB.) }\end{array}$ & Cattle stick & $\begin{array}{l}\text { Stomach problem, } \\
\text { malaria, snake bites, } \\
\text { leprosy, fever, ulcer, } \\
\text { dermal infection, } \\
\text { veneral diseases, } \\
\text { sterility, diarrhea, } \\
\text { headaches, wounds, } \\
\text { rheumatism fever, } \\
\text { pain, insanity, } \\
\text { aphrodiasac }\end{array}$ & $\begin{array}{l}\text { Leaves, } \\
\text { rootbark, } \\
\text { roots }\end{array}$ & Decoction & 0.03 & 50 & 0.8 & 2.66 & s.e, s.s, s.w & Lawal et al. 2010 \\
\hline $\begin{array}{l}\text { Cassia fistula L. } \\
\text { BDU } 04\end{array}$ & Fabaceae & Kasio & $\begin{array}{l}\text { Golden } \\
\text { shower tree }\end{array}$ & $\begin{array}{l}\text { Purgative, astringent, } \\
\text { vermifuge }\end{array}$ & Fruit & $\begin{array}{l}\text { Decoction, } \\
\text { tincture }\end{array}$ & 0.13 & 50 & 0.15 & 0.11 & s.e, s.s, s.w & $\begin{array}{l}\text { Lawal et al. 2010, } \\
\text { Soladoyeet al. } \\
2014\end{array}$ \\
\hline $\begin{array}{l}\text { Cassytha filiformis } \mathrm{L} \text {. } \\
\text { BDU } 100\end{array}$ & Lauraceae & Ngbuakpu (IK.) & $\begin{array}{l}\text { South sea } \\
\text { Islanders }\end{array}$ & $\begin{array}{l}\text { Lactation } \\
\text { suppressant, } \\
\text { vermifuge, jaundice, } \\
\text { infertility }\end{array}$ & $\begin{array}{l}\text { Leaves, } \\
\text { stem, } \\
\text { whole plant }\end{array}$ & $\begin{array}{l}\text { Decoction, } \\
\text { infusion, juice } \\
\text { extract }\end{array}$ & 0.05 & 60 & 0.2 & 0.4 & s.s, s.e & $\begin{array}{l}\text { Ajibesin et al. } \\
2008 \text { \& } 2012\end{array}$ \\
\hline $\begin{array}{l}\text { Ceiba petandra (L.) Gaertn. } \\
\text { BDU } 66\end{array}$ & Bombacaceae & Akpu-ogwu, Araba (I.) & $\begin{array}{l}\text { White silk } \\
\text { cotton tree }\end{array}$ & $\begin{array}{l}\text { Leprosy, tooth ache, } \\
\text { mouth problems, } \\
\text { conjunctivitis, eye } \\
\text { wounds, fever }\end{array}$ & $\begin{array}{l}\text { Leaves, } \\
\text { stembark, } \\
\text { root }\end{array}$ & $\begin{array}{l}\text { Decoction, baths, } \\
\text { compresses }\end{array}$ & 0.07 & 85 & 0.4 & 0.5 & s.w & Lawal et al. 2010 \\
\hline $\begin{array}{l}\text { Chenopodium ambrosioides L. } \\
\text { BDU } 68\end{array}$ & Chenopodiaceae & Arunpale $(Y)$. & $\begin{array}{l}\text { Sweet } \\
\text { pigweed }\end{array}$ & $\begin{array}{l}\text { Anti-hypertensive, } \\
\text { gonorrhea, syphilis, } \\
\text { laxative, febrifuge, } \\
\text { cough, tuberculosis }\end{array}$ & $\begin{array}{l}\text { Whole } \\
\text { plant }\end{array}$ & $\begin{array}{l}\text { Infusion, } \\
\text { mastication, } \\
\text { maceration }\end{array}$ & 0.1 & 60 & 0.4 & 0.4 & s.w & $\begin{array}{l}\text { Lawal et al. 2010, } \\
\text { Alade \& Ajibesin } \\
2017\end{array}$ \\
\hline $\begin{array}{l}\text { Chromolaena odorata (L.) R. M. } \\
\text { King \& Robinson } \\
\text { BDU } 167\end{array}$ & Asteraceae & $\begin{array}{l}\text { Nshegbuawom (EK.), } \\
\text { Obiarakara (I.), } \\
\text { Ukuro (O.) }\end{array}$ & $\begin{array}{l}\text { Siam weed, } \\
\text { Awolowo } \\
\text { weed }\end{array}$ & $\begin{array}{l}\text { Stomach upsets, } \\
\text { wounds, tooth aches, } \\
\text { malaria. Typhoid, } \\
\text { antimicrobial, } \\
\text { headache, dysentery, } \\
\text { hemorrhoids }\end{array}$ & $\begin{array}{l}\text { Leaves, leaf } \\
\text { sap }\end{array}$ & $\begin{array}{l}\text { Decoction, } \\
\text { maceration, } \\
\text { poultice, } \\
\text { compress }\end{array}$ & 0.17 & 88 & 0.5 & 0.26 & s.e, s.s & $\begin{array}{l}\text { Alade \& Ajibesin } \\
2017\end{array}$ \\
\hline $\begin{array}{l}\text { Chrysophyllum cainito L. } \\
\text { BDU } 171\end{array}$ & Sapotaceae & $\begin{array}{l}\text { Udala (I.), Agbalumo, } \\
\text { ebo (Y.) }\end{array}$ & $\begin{array}{l}\text { African star } \\
\text { apple }\end{array}$ & $\begin{array}{l}\text { Diabetes, larynx } \\
\text { inflammation, } \\
\text { pneumonia, angina, } \\
\text { diarrhoea, dysentery, } \\
\text { haemorrhage, } \\
\text { gonorrhoea, catarrh } \\
\text { of the bladder }\end{array}$ & $\begin{array}{l}\text { Fruit, } \\
\text { stembark }\end{array}$ & $\begin{array}{l}\text { Decoction, } \\
\text { infusion, } \\
\text { mastication }\end{array}$ & 0.13 & 96 & 0.5 & 0.34 & s.e, s.w & Lawal et al. 2010 \\
\hline $\begin{array}{l}\text { Citrullus colocynthis (L.) Schrad } \\
\text { BDU } 31\end{array}$ & Cucurbitaceae & Egusi & Bitter apple & $\begin{array}{l}\text { Syphilis, } \\
\text { stomachache, } \\
\text { laxative, skin disease }\end{array}$ & $\begin{array}{l}\text { Leaves, } \\
\text { fruits, seed } \\
\text { shell }\end{array}$ & $\begin{array}{l}\text { Decoction, } \\
\text { powder mixed } \\
\text { with palm oil }\end{array}$ & 0.19 & 84 & 0.2 & 0.11 & 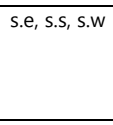 & $\begin{array}{l}\text { Ajibesin et al. } \\
2008 \& 2012, \\
\text { Soladoye et al. } \\
2014\end{array}$ \\
\hline
\end{tabular}


Ethnobotany Research and Applications

\begin{tabular}{|c|c|c|c|c|c|c|c|c|c|c|c|c|}
\hline $\begin{array}{l}\text { Citrus aurantifolia (Christm) } \\
\text { Swing. } \\
\text { BDU } 11\end{array}$ & Rutaceae & $\begin{array}{l}\text { Oromankirisi (I.), Otie- } \\
\text { Ogangan (U.) }\end{array}$ & Lime & $\begin{array}{l}\text { Catarrh, flu, } \\
\text { ringworm, eczema, } \\
\text { rheumatism, malaria, } \\
\text { impotency in men, as } \\
\text { mouth wash, stomach } \\
\text { trouble, fever }\end{array}$ & Fruit juice & $\begin{array}{l}\text { Aromatherapy, } \\
\text { baths, decoction, } \\
\text { infusion }\end{array}$ & 0.23 & 78 & 0.5 & 0.21 & s.e, s.s & $\begin{array}{l}\text { Alade \& Ajibesin } \\
\text { 2017, Iyama \& } \\
\text { Idu } 2015\end{array}$ \\
\hline $\begin{array}{l}\text { Citrus aurantium L. } \\
\text { BDU } 15\end{array}$ & Rutaceae & Osan-ijagoin (Y.) & $\begin{array}{l}\text { Sour / } \\
\text { Bitter } \\
\text { orange }\end{array}$ & Malaria & Fruits & Decoction & 0.07 & 85 & 0.05 & 0.07 & s.w & lyama \& Idu 2015 \\
\hline $\begin{array}{l}\text { Citrus limon (L.) Burm. F. } \\
\text { BDU } 60\end{array}$ & Rutaceae & $\begin{array}{l}\text { Lemonu (I.), Alimo- } \\
\text { negieghe (E.) }\end{array}$ & Lemon & $\begin{array}{l}\text { Digestive disorders, } \\
\text { diarrhea, ulcers, } \\
\text { excessive weight gain, } \\
\text { spots, scabs, wounds } \\
\text { scars, malaria, } \\
\text { Insect bites }\end{array}$ & Fruit juice & $\begin{array}{l}\text { Decoction, } \\
\text { tincture, infusion }\end{array}$ & 0.25 & 96 & 0.5 & 1.41 & s.e, s.s & Iyama \& Idu 2015 \\
\hline $\begin{array}{l}\text { Citrus medica L. } \\
\text { BDU } 40\end{array}$ & Rutaceae & & & Malaria & $\begin{array}{l}\text { Leaves, } \\
\text { fruits }\end{array}$ & Decoction & 0.1 & 70 & 0.05 & 0.05 & s.s, s.w & lyama \& Idu 2015 \\
\hline $\begin{array}{l}\text { Citrus paradisi Macf } \\
\text { BDU } 151\end{array}$ & Rutaceae & Osan-gerepu (Y.) & Grape & Malaria & Fruits & Decoction & 0.07 & 71 & 0.05 & 0.07 & s.w & Iyama \& Idu 2015 \\
\hline $\begin{array}{l}\text { Citrus sinensis Osbek } \\
\text { BDU } 06\end{array}$ & Rutaceae & Osan-mimo (Y.) & $\begin{array}{l}\text { Sweet } \\
\text { orange }\end{array}$ & $\begin{array}{l}\text { Ulcer, sore } \\
\text { tuberculosis } \\
\text { constipation, malaria, } \\
\text { fever, dysentery, } \\
\text { headache, tooth ache, } \\
\text { vermifuge }\end{array}$ & $\begin{array}{l}\text { Leaves, } \\
\text { fruits, } \\
\text { stembark }\end{array}$ & Decoction & 0.09 & 77 & 0.2 & 0.22 & s.w & $\begin{array}{l}\text { Alade \& Ajibesin } \\
\text { 2017, Ariwaodo } \\
\text { et al. 2012, Iyama } \\
\text { \& Idu 2015 }\end{array}$ \\
\hline $\begin{array}{l}\text { Clausena anisata (Will) Hook-f } \\
\text { ex Benth. } \\
\text { BDU } 130\end{array}$ & Rutaceae & Atapari, Obuko (Y.) & Clausena & Malaria & Leaves & Decoction & 0.07 & 71 & 0.05 & 0.07 & s.w & Iyama \& Idu 2015 \\
\hline $\begin{array}{l}\text { Cleistopholis patens (Benth.) } \\
\text { Engl. \& Diels } \\
\text { BDU } 05\end{array}$ & Annonaceae & $\begin{array}{l}\text { Out (E.) Ojo (I.), Orila, } \\
\text { Ator- angbo (Y.). }\end{array}$ & $\begin{array}{l}\text { Salt and oil } \\
\text { tree }\end{array}$ & $\begin{array}{l}\text { Malaria, fungal } \\
\text { infection }\end{array}$ & $\begin{array}{l}\text { Leaves, } \\
\text { stembark }\end{array}$ & $\begin{array}{l}\text { Decoction, } \\
\text { tincture }\end{array}$ & 0.03 & 83 & 0.1 & 0.33 & s.e, s.s, s.w & $\begin{array}{l}\text { Akwaji et al. } \\
\text { 2017, Iyama \& } \\
\text { Idu } 2015\end{array}$ \\
\hline $\begin{array}{l}\text { Clerodendrumpaniculatum } \mathrm{L} \text {. } \\
\text { BDU } 09\end{array}$ & Verbanaceae & Ora-ojola, Adabi (Y.) & $\begin{array}{l}\text { Garden } \\
\text { Quinine }\end{array}$ & Malaria & $\begin{array}{l}\text { Leaves, } \\
\text { roots }\end{array}$ & Decoction & 0.06 & 50 & 0.05 & 0.08 & s.w & lyama \& Idu 2015 \\
\hline $\begin{array}{l}\text { Cnestis ferruginea } \\
\text { DC. } \\
\text { BDU } 107\end{array}$ & Connaraceae & $\begin{array}{l}\text { Ukpo-ibieka (E.), } \\
\text { Usiereebua (EF.), Okpe- } \\
\text { nketa (IK.), Agwola (U.), } \\
\text { Akara-oje (Y.) }\end{array}$ & & $\begin{array}{l}\text { Eye infection, laxative, } \\
\text { fever, toothache, } \\
\text { haemorrhoid }\end{array}$ & $\begin{array}{l}\text { Leaves, } \\
\text { roots }\end{array}$ & Infusion & 0.13 & 96 & 0.25 & 0.19 & s.e, s.s, s.w & $\begin{array}{l}\text { Ariwaodo et al. } \\
2012\end{array}$ \\
\hline $\begin{array}{l}\text { Cochlospermum tinctorium A. } \\
\text { Rich } \\
\text { BDU } 93\end{array}$ & Cochlospermaceae & Feru (Y.), Gbutu (E.) & $\begin{array}{l}\text { Cotton } \\
\text { plant }\end{array}$ & Malaria & Roots & Decoction & 0.07 & 85 & 0.05 & 0.17 & s.s, s.w & Iyama \& Idu 2015 \\
\hline $\begin{array}{l}\text { Cocos nucifera L. } \\
\text { BDU } 76\end{array}$ & Araceae & Agbon (Y.) & Coconut & $\begin{array}{l}\text { Malaria, teething, } \\
\text { convulsions, skin } \\
\text { diseases, migraine, } \\
\text { fever, poison } \\
\text { antedote }\end{array}$ & $\begin{array}{l}\text { Stembark, } \\
\text { roots, fruits }\end{array}$ & Decoction & 0.15 & 90 & 0.4 & 0.26 & s.w & $\begin{array}{l}\text { Alade \& Ajibesin } \\
\text { 2017, Ariwaodo } \\
\text { et al. 2012, Iyama } \\
\text { \& Idu 2015 }\end{array}$ \\
\hline $\begin{array}{l}\text { Cola acuminata (P. Beauv.) } \\
\text { Schott and Endl. } \\
\text { BDU } 79\end{array}$ & Sterculiaceae & $\begin{array}{l}\text { Oji-hausa (I.), Obi- } \\
\text { abata (Y.) }\end{array}$ & Kola & Malaria & Stembark & Decoction & 0.07 & 85 & 0.05 & 0.07 & s.e, s.w & lyama \& Idu 2015 \\
\hline $\begin{array}{l}\text { Cola milleniï K. Schum. } \\
\text { BDU } 268\end{array}$ & Sterculiaceae & Obi-edun (Y.) & $\begin{array}{l}\text { Monkey } \\
\text { kola }\end{array}$ & $\begin{array}{l}\text { Anti-viral, ringworm, } \\
\text { scabies, gonorrhoea, } \\
\text { dysentery, opthalmia }\end{array}$ & $\begin{array}{l}\text { Leaves, } \\
\text { fruits }\end{array}$ & & 0.11 & 77 & 0.3 & 0.66 & s.w & Lawal et al. 2010 \\
\hline $\begin{array}{l}\text { Colocasia esculenta (L.) Schott } \\
\text { BDU } 131\end{array}$ & Araceae & Inodin; Ede (EK.) & $\begin{array}{l}\text { Cocoyam, } \\
\text { taro }\end{array}$ & Insect bites, sore & $\begin{array}{l}\text { Whole } \\
\text { plant }\end{array}$ & $\begin{array}{l}\text { Decoction, juice } \\
\text { extract }\end{array}$ & 0.03 & 50 & 0.1 & 0.33 & 5.5 & $\begin{array}{l}\text { Ajibesin et al. } \\
2008 \text { \& } 2012\end{array}$ \\
\hline
\end{tabular}


Ethnobotany Research and Applications

\begin{tabular}{|c|c|c|c|c|c|c|c|c|c|c|c|c|}
\hline $\begin{array}{l}\text { Combretum racemosum } \mathrm{P} . \\
\text { Beauv. } \\
\text { BDU } 87\end{array}$ & Combretaceae & $\begin{array}{l}\text { English cristmas rose } \\
\text { (IK.), Adallabanya (K.), } \\
\text { Okoso (E.), Alagame } \\
\text { (I.), Ogan (Y.) }\end{array}$ & $\begin{array}{l}\text { Christmas } \\
\text { rose }\end{array}$ & $\begin{array}{l}\text { Skin disease, } \\
\text { haemorrhoids, } \\
\text { convulsive coughing, } \\
\text { tuberculosis, tooth } \\
\text { ache }\end{array}$ & Leaves & Juice extract & 0.05 & 50 & 0.3 & 0.5 & s.e, s.s, s.w & $\begin{array}{l}\text { Ajibeesin et al. } \\
2008 \text { \& 2012, } \\
\text { Ugbogu \& } \\
\text { Chukwuma } 2019\end{array}$ \\
\hline $\begin{array}{l}\text { Cordia alliodora L. } \\
\text { BDU } 20\end{array}$ & Boraginaceae & Omo (Y.) & Salm-wood & Fungal infections & & & 0.14 & 92 & 0.05 & 0.03 & s.w & Lawal et al. 2010 \\
\hline $\begin{array}{l}\text { Corchorus olitorus } \\
\text { BDU } 07\end{array}$ & Tiliaceae & Ahihiara (I.) & $\begin{array}{l}\text { Jute } \\
\text { vegetable }\end{array}$ & $\begin{array}{l}\text { Irregular } \\
\text { menstruation, fever, } \\
\text { malaria }\end{array}$ & $\begin{array}{l}\text { Leaves+ } \\
\text { rubber } \\
\text { plant leaves }\end{array}$ & $\begin{array}{l}\text { Infusion, cold } \\
\text { water extract }\end{array}$ & 0.05 & 60 & 0.15 & 0.3 & s.e & $\begin{array}{l}\text { lyama \& Idu } \\
\text { 2015, obute } 2005 \\
\& 2007\end{array}$ \\
\hline $\begin{array}{l}\text { Corylus avellana L. } \\
\text { BDU } 104\end{array}$ & Betulaceae & & Hazel & $\begin{array}{l}\text { Hemorrhoid } \\
\text { menstrual disorder, } \\
\text { bleeding }\end{array}$ & $\begin{array}{l}\text { Leaves, } \\
\text { flowers }\end{array}$ & Decoction & 0.06 & 66 & 0.15 & 0.3 & s.s & $\begin{array}{l}\text { Ajibesin et al } \\
.2008 \text { \& } 2012\end{array}$ \\
\hline $\begin{array}{l}\text { Costus afer Ker. } \\
\text { BDU } 132\end{array}$ & Costaceae & $\begin{array}{l}\text { Okpete }(\mathrm{U} .) \text {, Ireke- } \\
\text { omode }(\mathrm{Y} .)\end{array}$ & $\begin{array}{l}\text { Bush cane, } \\
\text { common } \\
\text { ginger lilly }\end{array}$ & $\begin{array}{l}\text { Malaria, nausea, } \\
\text { stomachache, } \\
\text { aphrodiasac }\end{array}$ & $\begin{array}{l}\text { Leaves, } \\
\text { stembark }\end{array}$ & Decoction & 0.1 & 80 & 0.2 & 0.2 & s.s, s.w & $\begin{array}{l}\text { lyama \& Idu } \\
\text { 2015, Ariwaodo } \\
\text { et al. 2012, Ajuru } \\
2018\end{array}$ \\
\hline $\begin{array}{l}\text { Costus lucanusianus Braun \& } \\
\text { Schum } \\
\text { BDU } 19\end{array}$ & Costaceae & Piri-ngwo (IK.) & Bush cane & Measles, eye defects & Stembark & $\begin{array}{l}\text { Baths, } \\
\text { compresses, } \\
\text { decoction }\end{array}$ & 0.18 & 80 & 0.1 & 0.05 & s.e & Obute \& Adubor \\
\hline $\begin{array}{l}\text { Crateva adansonii DC. } \\
\text { BDU48 }\end{array}$ & Capparaceae & Amakarode (K.) & $\begin{array}{l}\text { Garlic pear } \\
\text { tree }\end{array}$ & $\begin{array}{l}\text { Rheumatism, malaria, } \\
\text { infertility, } \\
\text { stomachache }\end{array}$ & $\begin{array}{l}\text { Leaves, } \\
\text { stembark }\end{array}$ & $\begin{array}{l}\text { Decoction, } \\
\text { poultices }\end{array}$ & 0.06 & 75 & 0.2 & 0.33 & s.s & $\begin{array}{l}\text { Ajibesin } e t \text { al. } \\
2008 \& 2012\end{array}$ \\
\hline $\begin{array}{l}\text { Crotalaria retusa L. } \\
\text { BDU } 120\end{array}$ & Fabaceae & Koropo, Alatunse (Y.) & $\begin{array}{l}\text { Rattle pea, } \\
\text { Rattle Box }\end{array}$ & Malaria & Leaves, root & Decoction & 0.03 & 50 & 0.1 & 0.16 & s.w & Iyama \& Idu 2015 \\
\hline $\begin{array}{l}\text { Croton lobatus L. } \\
\text { BDU71 }\end{array}$ & Euphorbiaceae & Okwe (I.), Ajeofole (Y.) & $\begin{array}{l}\text { Lobed } \\
\text { croton }\end{array}$ & $\begin{array}{l}\text { Skin disease, } \\
\text { rheumatism,scorpions } \\
\text { ting, stomachache }\end{array}$ & Leaves & $\begin{array}{l}\text { Decoction, } \\
\text { poultice }\end{array}$ & 0.14 & 75 & 0.2 & 0.14 & s.e, s.w & $\begin{array}{l}\text { Ajibesin et al. } \\
2008 \& 2012\end{array}$ \\
\hline $\begin{array}{l}\text { Croton zambesicus Müll. -Arg. } \\
\text { BDU } 78\end{array}$ & Euphorbiaceae & $\begin{array}{l}\text { Ajekobale (Y.), Alele } \\
\text { (O.) }\end{array}$ & & Dysentery, diarrhea & Leaves & Decoction & 0.06 & 75 & 0.1 & 0.16 & s.s, s.w & $\begin{array}{l}\text { Ajibesin et al. } \\
2008 \& 2012\end{array}$ \\
\hline $\begin{array}{l}\text { Cryptolepis sanguinolenta } \\
\text { (Lindl) Schtlr } \\
\text { BDU } 159\end{array}$ & Periplocaceae & Gangamau & $\begin{array}{l}\text { Cryptole- } \\
\text { pis }\end{array}$ & Malaria & Stembark & Decoction & 0.02 & 75 & 0.1 & 0.3 & s.s & lyama \& Idu 2015 \\
\hline $\begin{array}{l}\text { Curcuma longa L. } \\
\text { BDU } 83\end{array}$ & Zingiberaceae & $\begin{array}{l}\text { Buru, Simogo (U.), } \\
\text { Laali-pupa (Y.) }\end{array}$ & Turmeric & Malaria & Rhizome & $\begin{array}{l}\text { Decoction, } \\
\text { infusion }\end{array}$ & 0.03 & 83 & 0.1 & 0.16 & s.s, s.w & Iyama \& Idu 2015 \\
\hline $\begin{array}{l}\text { Cucumis sativus L. } \\
\text { BDU } 247\end{array}$ & Cucuribitaceae & & Cucumber & Arthritis, pain & Fruits & Juice extract & 0.07 & 85 & 0.1 & 0.14 & s.w & Akwaji et al. 2017 \\
\hline $\begin{array}{l}\text { Cucurbita maxima Duch. } \\
\text { BDU } 198\end{array}$ & Cucuribitaceae & Akumocha (IK.) & Pumpkin & Skin disease & Leaves & Juice extract & 0.1 & 60 & 0.05 & 0.05 & s.s & $\begin{array}{l}\text { Ajibesin et al. } \\
2008 \& 2012\end{array}$ \\
\hline $\begin{array}{l}\text { Cymbopogon citratus (DC.) } \\
\text { Stapf. } \\
\text { BDU } 217\end{array}$ & Poaceae & $\begin{array}{l}\text { Obiete/Ebitien (U.), } \\
\text { Ewe-tea, Kooko-oba } \\
\text { (Y) }\end{array}$ & $\begin{array}{l}\text { Lemon } \\
\text { grass }\end{array}$ & $\begin{array}{l}\text { Typhoid fever, cough, } \\
\text { malaria pneumonia, } \\
\text { as an astringent, } \\
\text { diuretic, antiseptic }\end{array}$ & $\begin{array}{l}\text { Leaves } \\
\text { +bark } \\
\text { of } A \text { Istoniab } \\
\text { oonei } \\
\text { grapefruits, } \\
\text { pineapple, } \\
\text { pawpaw } \\
\text { and garlic }\end{array}$ & Decoction & 0.25 & 96 & 0.4 & 0.14 & s.s, s.w & lyama \& Idu 2015 \\
\hline $\begin{array}{l}\text { Dacryoides edulis (D. Don) Lam. } \\
\text { BDU s129 }\end{array}$ & Burreraceae & $\begin{array}{l}\text { Ube }(I .) \text { Elemi }(Y .), \\
\text { Eben (I.) }\end{array}$ & Native pear & $\begin{array}{l}\text { Skin } \\
\text { parasites/disease, } \\
\text { hypertension, cough, } \\
\text { heat conditions, } \\
\text { malaria }\end{array}$ & $\begin{array}{l}\text { Leaves, } \\
\text { stem resins, } \\
\text { fruits }\end{array}$ & Decoction & 0.2 & 95 & 0.3 & 0.13 & s.e, s.s, s.w & Iyama \& Idu 2015 \\
\hline $\begin{array}{l}\text { Daucus carota L } \\
\text { BDU14 }\end{array}$ & Apiaceae & Nsoro (IK.) & Wild carrot & Eye infection & Root & Decoction & 0.11 & 95 & 0.05 & 0.05 & s.s & $\begin{array}{l}\text { Ajibesin et al. } \\
2008 \& 2012\end{array}$ \\
\hline
\end{tabular}


Ethnobotany Research and Applications

\begin{tabular}{|c|c|c|c|c|c|c|c|c|c|c|c|c|}
\hline $\begin{array}{l}\text { Dalbergia lacteal Vatke } \\
\text { BDU } 152\end{array}$ & Fabaceae & Ojjij, abinrere (Y.) & Dalbergia & $\begin{array}{l}\text { Sore throat, pimples, } \\
\text { skin disease }\end{array}$ & Stem & & 0.03 & 83 & 0.15 & 0.5 & s.w & Lawal et al. 2010 \\
\hline $\begin{array}{l}\text { Dalbergia latifolia Roxb. } \\
\text { BDU } 244\end{array}$ & Fabaceae & Ogun-aja (Y.) & $\begin{array}{l}\text { Indian } \\
\text { rosewood }\end{array}$ & Yellow fever & & & 0.05 & 60 & 0.05 & 0.1 & s.w & Lawal et al. 2010 \\
\hline $\begin{array}{l}\text { Daniella ogea Harms. } \\
\text { BDU } 453\end{array}$ & Fabaceae & Iyaa (Y.) & & $\begin{array}{l}\text { Nerves soothing, back } \\
\text { pain }\end{array}$ & & & 0.06 & 75 & 0.1 & 0.16 & s.w & Lawal et al. 2010 \\
\hline Dennettia tripetala Bak. BDU 61 & Annonaceae & Nimi (I.), Ata-igberi (Y.) & $\begin{array}{l}\text { Pepper } \\
\text { Fruit }\end{array}$ & Malaria, colds & $\begin{array}{l}\text { Leaves, } \\
\text { Fruits }\end{array}$ & $\begin{array}{l}\text { Decoction, } \\
\text { mastication }\end{array}$ & 0.23 & 93 & 0.1 & 0.04 & s.e, s.w & lyama \& Idu 2015 \\
\hline $\begin{array}{l}\text { Dialium guineense Willd. } \\
\text { BDU } 27\end{array}$ & Fabaceae & Ugbe-him (IK.) & $\begin{array}{l}\text { Velvet } \\
\text { Tamarind }\end{array}$ & $\begin{array}{l}\text { Malaria, diarrhea, } \\
\text { stomachache, } \\
\text { toothache }\end{array}$ & Leaves & Infusion & 0.11 & 95 & 0.2 & 0.18 & s.s & Akwaji et al. 2017 \\
\hline $\begin{array}{l}\text { Diodia scandens Sw } \\
\text { BDU } 96\end{array}$ & Rubiaceae & Onaedi (I.) & & $\begin{array}{l}\text { After birth womb } \\
\text { cleansing in females, } \\
\text { vermifuge }\end{array}$ & $\begin{array}{l}\text { Leaves + } \\
\text { Napoleonai } \\
\text { mperialis }\end{array}$ & Infusion & 0.1 & 60 & 0.1 & 0.1 & s.e & $\begin{array}{l}\text { Obute } 2005 \& \text { \& } \\
2007\end{array}$ \\
\hline $\begin{array}{l}\text { Dioscorea dumentorum (Knuth) } \\
\text { Pax } \\
\text { BDU } 202\end{array}$ & Dioscoreaceae & $\begin{array}{l}\text { Ona ochao (I.),E suru- } \\
\text { igbo (Y.) }\end{array}$ & $\begin{array}{l}\text { African } \\
\text { bitter Yam }\end{array}$ & $\begin{array}{l}\text { Malaria, abdominal } \\
\text { pain }\end{array}$ & Leaves & powdered & 0.03 & 83 & 0.1 & 0.33 & s.e, s.w & lyama \& Idu 2015 \\
\hline $\begin{array}{l}\text { Dioscorea rotundata Poir } \\
\text { BDU } 98\end{array}$ & Dioscoreaceae & Fingi (K.) & $\begin{array}{l}\text { White yam, } \\
\text { West } \\
\text { African } \\
\text { yam }\end{array}$ & Burns, skin disease & Leaves & Infusion & 0.02 & 75 & 0.1 & 0.5 & s.s & $\begin{array}{l}\text { Ajibesin et al. } \\
2008 \& 2012\end{array}$ \\
\hline $\begin{array}{l}\text { Distemonanthus benthamianus } \\
\text { Baill. } \\
\text { BDU } 62\end{array}$ & Fabaceae & $\begin{array}{l}\text { Anyarthan (B.), Osashi } \\
\text { (IK.) }\end{array}$ & $\begin{array}{l}\text { African } \\
\text { satinwood }\end{array}$ & $\begin{array}{l}\text { Skin disease, boils, } \\
\text { malaria, tonic for } \\
\text { pregnant women }\end{array}$ & Stembark & $\begin{array}{l}\text { Decoction with } \\
\text { Pterocarpus } \\
\text { erinaceus, } \\
\text { mastication, } \\
\text { poultice }\end{array}$ & 0.06 & 66 & 0.2 & 0.33 & s.s & $\begin{array}{l}\text { Ugbogu \& } \\
\text { Chukwuma } 2019\end{array}$ \\
\hline $\begin{array}{l}\text { Dracaena arborea (Wild.) BDU } \\
30\end{array}$ & Dracaenaceae & Odo (IK.) & $\begin{array}{l}\text { African } \\
\text { dragon } \\
\text { tree }\end{array}$ & Stomachache, boils & $\begin{array}{l}\text { Leaves, } \\
\text { stembark }\end{array}$ & Infusion, poultice & 0.11 & 95 & 0.1 & 0.09 & s.s & Akwaji et al. 2017 \\
\hline $\begin{array}{l}\text { Elaeis guineensis Jacq. } \\
\text { BDU } 01\end{array}$ & Araceae & $\begin{array}{l}\text { Nkwu, akwu (I.), } \\
\text { Obaekpe (U.), Ope, } \\
\text { Eyin (Y.) }\end{array}$ & Oil palm & $\begin{array}{l}\text { Antidote for poison, } \\
\text { skin troubles, } \\
\text { convulsions, colds, } \\
\text { persistent coughs, } \\
\text { fibroids, malaria, } \\
\text { dysmenorrheal }\end{array}$ & $\begin{array}{l}\text { Oil, unripe } \\
\text { kernel, } \\
\text { leaves }\end{array}$ & $\begin{array}{l}\text { Aromatherapy, } \\
\text { decoction, } \\
\text { mastication, } \\
\text { syrups }\end{array}$ & 0.22 & 99 & 0.4 & 0.18 & s.s, s.w, s.e & $\begin{array}{l}\text { lyama \& Idu } \\
2015 \text {, Lawal et al. } \\
2010\end{array}$ \\
\hline $\begin{array}{l}\text { Eleusine indica (Linn) Gaertn. } \\
\text { BDU } 33\end{array}$ & Poaceae & Ichite (I.) & $\begin{array}{l}\text { Goose } \\
\text { grass, } \\
\text { wiregrass }\end{array}$ & $\begin{array}{l}\text { Anti-inflammatory, } \\
\text { convulsions, cough }\end{array}$ & Roots & & 0.1 & 95 & 0.15 & 0.15 & s.e & Akwaji et al. 2017 \\
\hline $\begin{array}{l}\text { Emilia coccinea (Sims) G. Don. } \\
\text { BDU } 247\end{array}$ & Asteraceae & Ntiene (EK.) & $\begin{array}{l}\text { Tassel } \\
\text { flower }\end{array}$ & $\begin{array}{l}\text { Ear and eye infection, } \\
\text { Measles, skin } \\
\text { diseases, sores }\end{array}$ & Leaves & Juice extract & 0.09 & 88 & 0.3 & 0.27 & s.s & $\begin{array}{l}\text { Ajibesin et al. } \\
20082012\end{array}$ \\
\hline $\begin{array}{l}\text { Emilia sonchifolia (L.) DC } \\
\text { BDU } 02\end{array}$ & Asteraceae & $\begin{array}{l}\text { Ogbunizu (I.), } \\
\text { Odundunodo (Y.) }\end{array}$ & $\begin{array}{l}\text { Yellow } \\
\text { tassel } \\
\text { flower }\end{array}$ & $\begin{array}{l}\text { Anticoagulant in } \\
\text { wound treatment, } \\
\text { malaria }\end{array}$ & $\begin{array}{l}\text { Leaves, } \\
\text { whole plant }\end{array}$ & $\begin{array}{l}\text { Decoction, } \\
\text { topical } \\
\text { application of } \\
\text { leaf juice extract. }\end{array}$ & 0.05 & 70 & 0.1 & 0.2 & s.e, s.w & Iyama \& Idu 2015 \\
\hline Enantia chlorantha Ol BDU 03 & Annonaceae & Awopa (Y.) & $\begin{array}{l}\text { African } \\
\text { yellow } \\
\text { wood }\end{array}$ & Malaria & Stembark & $\begin{array}{l}\text { Decoction, } \\
\text { infusion, } \\
\text { powdered }\end{array}$ & 0.14 & 92 & 0.05 & 0.04 & s.w & lyama \& Idu 2015 \\
\hline $\begin{array}{l}\text { Ertythrinia abbyssinica L. } \\
\text { BDU } 84\end{array}$ & Fabaceae & $\begin{array}{l}\text { Ologbosere, Lakale, } \\
\text { Majiriya (Y.) }\end{array}$ & & Yellow fever & & & 0.03 & 66 & 0.05 & 0.16 & s.w & Lawal et al. 2010 \\
\hline $\begin{array}{l}\text { Erythrinia senegalensis D.C } \\
\text { BDU } 159\end{array}$ & Fabaceae & $\begin{array}{l}\text { Ologbo-sere (Y.), } \\
\text { Onugobi (IK.), Echichi } \\
\text { (EK.) }\end{array}$ & Parrot tree & $\begin{array}{l}\text { Malaria, } \\
\text { stomachaches, } \\
\text { jaundice, gonorrhea, } \\
\text { ulcers }\end{array}$ & $\begin{array}{l}\text { Leaves, } \\
\text { roots }\end{array}$ & $\begin{array}{l}\text { Decoction, leaf } \\
\text { juice extract }\end{array}$ & 0.22 & 90 & 0.3 & 0.11 & s.s, s.w & $\begin{array}{l}\text { Akwaji et al. } \\
\text { 2017, Ajbesin et } \\
\text { al. 2008\& 2012, } \\
\text { lyama \& Idu } 2015\end{array}$ \\
\hline
\end{tabular}


Ethnobotany Research and Applications

\begin{tabular}{|c|c|c|c|c|c|c|c|c|c|c|c|c|}
\hline $\begin{array}{l}\text { Equisetum arvense L. } \\
\text { BDU } 168\end{array}$ & Equisaceae & Oruetemi (K.) & Horse Tail & $\begin{array}{l}\text { Asthma, cough, } \\
\text { urinary tract infection }\end{array}$ & Leaves & $\begin{array}{l}\text { Decoction, } \\
\text { infusion }\end{array}$ & 0.02 & 75 & 0.15 & 0.8 & s.s & $\begin{array}{l}\text { Ajibesin } \text { et al. } \\
2008 \& 2012 \\
\end{array}$ \\
\hline $\begin{array}{l}\text { Eucalyptus camaldulensis Dehn } \\
\text { BDU } 157\end{array}$ & Myrtaceae & & Eucalyptus & $\begin{array}{l}\text { Malaria, colds, } \\
\text { convulsions, } \\
\text { respiratory disorders, } \\
\text { allergies }\end{array}$ & $\begin{array}{l}\text { Leaves, } \\
\text { stembark }\end{array}$ & Decoction & 0.09 & 88 & 0.3 & 0.27 & s.e, s.s, s.w & lyama \& Idu 2015 \\
\hline $\begin{array}{l}\text { Euphorbia hirta L. } \\
\text { BDU } 189\end{array}$ & Euphorbiaceae & $\begin{array}{l}\text { ogwuasma, ahihiaugwa } \\
\text { (I.), Okwungwo (IK.) }\end{array}$ & $\begin{array}{l}\text { Australian } \\
\text { asthma } \\
\text { plant, } \\
\text { garden } \\
\text { spurge, } \\
\text { spurge } \\
\text { weed }\end{array}$ & $\begin{array}{l}\text { Asthma, catarrh, } \\
\text { eczema, immune } \\
\text { builder, malaria }\end{array}$ & Leaves & $\begin{array}{l}\text { Decoction, } \\
\text { topical } \\
\text { application, } \\
\text { aromatherapy }\end{array}$ & 0.14 & 97 & 0.3 & 0.18 & s.e, s.s & $\begin{array}{l}\text { Akwaji et al. } \\
\text { 2017, lyama \& } \\
\text { Idu } 2015\end{array}$ \\
\hline $\begin{array}{l}\text { Ficus asperifolia Miq. } \\
\text { BDU } 51\end{array}$ & Moraceae & $\operatorname{lpin}(Y)$. & & Hypertension & & & 0.02 & 50 & 0.05 & 0.3 & s.w & Lawal et al. 2010 \\
\hline $\begin{array}{l}\text { Ficus exasperate Vahl } \\
\text { BDU } 167\end{array}$ & Moraceae & Epin (Y.) & $\begin{array}{l}\text { Sandpaper } \\
\text { tree }\end{array}$ & $\begin{array}{l}\text { Malaria, female } \\
\text { infertility }\end{array}$ & Leaves & Decoction & 0.06 & 91 & 0.1 & 0.16 & s.w & $\begin{array}{l}\text { lyama \& Idu } \\
\text { 2015, Soladoyeet } \\
\text { al. 2014 }\end{array}$ \\
\hline $\begin{array}{l}\text { Fluerya aestuans (L) Gaud ex } \\
\text { mig } \\
\text { BDU } 112\end{array}$ & Urticaceae & Ipe-eri (Y.) & $\begin{array}{l}\text { Old woman } \\
\text { smokes } \\
\text { tobacco }\end{array}$ & Malaria & Roots & Decoction & 0.03 & 83 & 0.05 & 0.16 & s.w & lyama \& Idu 2015 \\
\hline $\begin{array}{l}\text { Foeniculum vulgare Mill. } \\
\text { BDU } 122\end{array}$ & Apiaceae & Icheje (IK.) & Fennel & $\begin{array}{l}\text { Eye infection, } \\
\text { cough }\end{array}$ & Seeds & $\begin{array}{l}\text { Infusion, drops, } \\
\text { poultice }\end{array}$ & 0.06 & 66 & 0.1 & 0.16 & 5.5 & $\begin{array}{l}\text { Ajibesin et al. } \\
2008 \& 2012\end{array}$ \\
\hline $\begin{array}{l}\text { Funtumia africana (Benth). Stapf. } \\
\text { BDU } 127\end{array}$ & Apocynaceae & Ako-ire (Y.) & $\begin{array}{l}\text { Male } \\
\text { Funtum }\end{array}$ & Malaria & Roots & Infusion & 0.13 & 96 & 0.05 & 0.04 & s.w & Iyama \& Idu 2015 \\
\hline $\begin{array}{l}\text { Funtumia elastica (Preuss) Stapf } \\
\text { BDU 179 }\end{array}$ & Apocynaceae & Mini-eme (IK.) & $\begin{array}{l}\text { Bush } \\
\text { rubber tree }\end{array}$ & $\begin{array}{l}\text { Hemorrhoids, } \\
\text { jaundice, impotence }\end{array}$ & Stembark & Infusion, poultice & 0.08 & 94 & 0.15 & 0.18 & s.s & Akwaji et al. 2017 \\
\hline $\begin{array}{l}\text { Garcinia kola Heckel } \\
\text { BDU } 93\end{array}$ & Clusiaceae & $\begin{array}{l}\text { Akuilu, agbuilu (I.), Aka } \\
\text { (U.), Orogbo (Y.) }\end{array}$ & Bitter cola & $\begin{array}{l}\text { Bronchitis, throat } \\
\text { infection, malaria, } \\
\text { asthma, cough }\end{array}$ & $\begin{array}{l}\text { Seeds, } \\
\text { roots }\end{array}$ & $\begin{array}{l}\text { Infusion, } \\
\text { mastication }\end{array}$ & 0.15 & 80 & 0.3 & 0.2 & s.s, s.e, s.w & $\begin{array}{l}\text { Alade \& Ajibesin } \\
\text { 2017, Soladoyeet } \\
\text { al. } 2014\end{array}$ \\
\hline $\begin{array}{l}\text { Gliricidia sepium Jacq. } \\
\text { BDU } 97\end{array}$ & Fabaceae & Agunmaniye $(Y)$. & & Rheumatism & & & 0.07 & 50 & 005 & 0.07 & s.w & Lawal et al. 2010 \\
\hline $\begin{array}{l}\text { Glycine max L. } \\
\text { BDU } 79\end{array}$ & Fabaceae & Mmanuagwa (EK.) & Soy bean & $\begin{array}{l}\text { Measles, } \\
\text { hepatitis,diabetes }\end{array}$ & Seeds & $\begin{array}{l}\text { Decoction, } \\
\text { poultices of oil } \\
\text { extract }\end{array}$ & 0.06 & 50 & 0.15 & 0.3 & s.s & $\begin{array}{l}\text { Ajibesin et al. } \\
2008 \& 2012\end{array}$ \\
\hline $\begin{array}{l}\text { Glyphaea brevis (Spreng.) } \\
\text { Monach } \\
\text { BDU } 201\end{array}$ & Tiliaceae & Atori (Y.) & $\begin{array}{l}\text { Masque- } \\
\text { rade stick }\end{array}$ & $\begin{array}{l}\text { Malaria, female } \\
\text { infertility, fever, } \\
\text { diarrhea, tooth ache }\end{array}$ & $\begin{array}{l}\text { Leaves, } \\
\text { stembark }\end{array}$ & Decoction & 0.02 & 50 & 0.3 & 1.3 & s.w & $\begin{array}{l}\text { Ariwaodo et al. } \\
\text { 2012, lyama \& } \\
\text { Idu 2015, Lawal } \\
\text { et al. 2010, } \\
\text { Soladoyeet al. } \\
2014\end{array}$ \\
\hline $\begin{array}{l}\text { Gmelina arborea Roxb. } \\
\text { BDU } 41\end{array}$ & Verbenaceae & Igi Melina & Beechwood & $\begin{array}{l}\text { High blood pressure, } \\
\text { diarrhoea }\end{array}$ & & & 0.09 & 66 & 0.1 & 0.11 & s.w & Lawal et al. 2010 \\
\hline $\begin{array}{l}\text { Gongronema latifolium Benth. } \\
\text { BDU126 }\end{array}$ & Asclepiadaceae & Utazi (I.) & $\begin{array}{l}\text { Amaranth } \\
\text { globe }\end{array}$ & $\begin{array}{l}\text { Malaria, womb } \\
\text { stabilization after } \\
\text { birthing, diabetes, } \\
\text { hypertension, } \\
\text { vermifuge, anaemia }\end{array}$ & Leaves & $\begin{array}{l}\text { Decoction with } \\
\text { Citrus } \\
\text { aurantifolia } \\
\text { juice and Pine } \\
\text { juice, juice } \\
\text { extract }\end{array}$ & 0.13 & 96 & 0.4 & 0.26 & s.e & $\begin{array}{l}\text { lyama \& Idu } \\
\text { 2015, Soladoyeet } \\
\text { al. } 2014\end{array}$ \\
\hline $\begin{array}{l}\text { Gossypium barbadense L. } \\
\text { BDU119 }\end{array}$ & Malvaceae & Ewe-owu (Y.) & $\begin{array}{l}\text { West } \\
\text { Indian } \\
\text { Cotton }\end{array}$ & $\begin{array}{l}\text { Dysmenorrheal, } \\
\text { malaria }\end{array}$ & Leaves & Decoction & 0.07 & 85 & 0.1 & 0.14 & s.w & Iyama \& Idu 2015 \\
\hline $\begin{array}{l}\text { Gossypium hirsutum L. } \\
\text { BDU } 115\end{array}$ & Malvaceae & Ela-owu (Y.) & Cotton & Malaria & Leaves & Decoction & 0.03 & 50 & 0.05 & 0.16 & s.w & $\begin{array}{l}\text { lyama \& Idu } \\
2015 s\end{array}$ \\
\hline
\end{tabular}


Ethnobotany Research and Applications

\begin{tabular}{|c|c|c|c|c|c|c|c|c|c|c|c|c|}
\hline $\begin{array}{l}\text { Harungana madagascariensis } \\
\text { Lamex Poir } \\
\text { BDU } 116\end{array}$ & Hypericaceae & $\begin{array}{l}\text { Otori (I.), Amuje, } \\
\text { Asunje (Y.), Uwara (U.) }\end{array}$ & $\begin{array}{l}\text { Dragon's } \\
\text { blood tree }\end{array}$ & $\begin{array}{l}\text { Skin disease, itches, } \\
\text { leprous spots malaria }\end{array}$ & $\begin{array}{l}\text { Plant sap, } \\
\text { leaves, } \\
\text { stembark }\end{array}$ & $\begin{array}{l}\text { Decoction, } \\
\text { topical } \\
\text { application }\end{array}$ & 0.15 & 80 & 0.2 & 0.13 & s.e, s.s, s.w & lyama \& Idu 2015 \\
\hline $\begin{array}{l}\text { Heliotropium indicum L } \\
\text { BDU110 }\end{array}$ & Boraginaceae & Akuko (Y.), Azu (EK.) & $\begin{array}{l}\text { Heliotrope, } \\
\text { Cock's } \\
\text { comb }\end{array}$ & $\begin{array}{l}\text { Malaria, convulsion, } \\
\text { toothache, Scorpion } \\
\text { sting, insect bites, } \\
\text { boils }\end{array}$ & $\begin{array}{l}\text { Leaves, } \\
\text { whole plant }\end{array}$ & $\begin{array}{l}\text { Decoction } \\
\text { infusion }\end{array}$ & 0.02 & 50 & 0.3 & 1.5 & s.s, s.w & lyama \& Idu 2015 \\
\hline $\begin{array}{l}\text { Hyptis suaveolens (L.) Poit } \\
\text { BDU } 138\end{array}$ & Lamiaceae & Jogbo (Y.) & Hyptis & Malaria & Leaves & Decoction & 0.05 & 70 & 0.05 & 0.1 & s.w & lyama \& Idu 2015 \\
\hline $\begin{array}{l}\text { Icacina trichanta Oliv. } \\
\text { BDU } 137\end{array}$ & Icacinaceae & $\begin{array}{l}\text { Mbia (IK.), Gbegbe (Y.), } \\
\text { Osun (B.) }\end{array}$ & Icacina & $\begin{array}{l}\text { Malaria, asthma, } \\
\text { rheumatism, } \\
\text { toothache, purgative }\end{array}$ & Leaves, fruit & Decoction, juice & 0.11 & 95 & 0.3 & 0.22 & s.s, s.w & $\begin{array}{l}\text { Ariwaodo et al. } \\
\text { 2012, lyama \& } \\
\text { Idu 2015, } \\
\text { Ugbogu \& } \\
\text { Chukwuma } 2019\end{array}$ \\
\hline $\begin{array}{l}\text { Ipomoea batatas } \mathrm{L} . \\
\text { BDU } 121\end{array}$ & Convolvulaceae & oboribo & $\begin{array}{l}\text { Sweet } \\
\text { Potato }\end{array}$ & Vitamin deficiency & $\begin{array}{l}\text { Whole } \\
\text { tuber }\end{array}$ & Meals & 0.03 & 66 & 0.05 & 0.16 & $\begin{array}{l}\text { s.e, s.s., s.w } \\
\end{array}$ & Oke et al.1999 \\
\hline $\begin{array}{l}\text { Ipomoea Involucrata P. Beauv } \\
\text { BDU } 129\end{array}$ & Convolvulaceae & $\begin{array}{l}\text { Fifilori (K.), Mgban-ala } \\
\text { (I.), Alukorese (Y.) }\end{array}$ & & $\begin{array}{l}\text { Asthma, malaria, } \\
\text { rheumatism }\end{array}$ & $\begin{array}{l}\text { Leaves, } \\
\text { stem }\end{array}$ & $\begin{array}{l}\text { Decoction, } \\
\text { infusion }\end{array}$ & 0.06 & 50 & 0.15 & 0.03 & s.e, s.s, s.w & $\begin{array}{l}\text { Ariwaodo et al } \\
\text { 2012, Ajibesin et } \\
\text { al. } 2008 \text { \& } 2012\end{array}$ \\
\hline $\begin{array}{l}\text { Ipomoea mauritiana Jacq. } \\
\text { BDU } 146\end{array}$ & Convolvulaceae & $\begin{array}{l}\text { Mgban-ala (O.), Atewo } \\
\text { (Y.) }\end{array}$ & $\begin{array}{l}\text { Morning } \\
\text { glory, Palm } \\
\text { of monkey } \\
\text { hand }\end{array}$ & Asthma & Roots & Decoction & 0.06 & 50 & 0.05 & 0.08 & s.s, s.w & $\begin{array}{l}\text { Ajibesin et al. } \\
2008 \& 2012\end{array}$ \\
\hline $\begin{array}{l}\text { Irvingia gabonensis (Aubry- } \\
\text { LeComteex O'Rorke) Bail. } \\
\text { BDU } 189\end{array}$ & Invingaceae & Agbono & $\begin{array}{l}\text { West } \\
\text { African } \\
\text { bush } \\
\text { mango }\end{array}$ & $\begin{array}{l}\text { Stomachache, skin } \\
\text { disease, infertility, as } \\
\text { vermifuge }\end{array}$ & $\begin{array}{l}\text { Leaves, } \\
\text { stembark, } \\
\text { seeds }\end{array}$ & $\begin{array}{l}\text { Decoction, } \\
\text { infusion, } \\
\text { poultice }\end{array}$ & 0.11 & 86 & 0.2 & 0.18 & s.s, s.w & $\begin{array}{l}\text { Ariwaodo et al. } \\
\text { 2012, Akwaji et } \\
\text { al. } 2017\end{array}$ \\
\hline $\begin{array}{l}\text { Jatropha curcas J.L. Ellis \& Saroja } \\
\text { BDU } 149\end{array}$ & Euphorbiaceae & $\begin{array}{l}\text { Oluluidu (O.), Ishakpa } \\
\text { (U.), Lapalapa-funfun } \\
\text { (Y.) }\end{array}$ & $\begin{array}{l}\text { Boundary } \\
\text { stick, } \\
\text { Physic nut, } \\
\text { Pig nut }\end{array}$ & $\begin{array}{l}\text { Anaemia, malaria, as } \\
\text { blood booster, }\end{array}$ & $\begin{array}{l}\text { Leaves, } \\
\text { stembark, } \\
\text { roots }\end{array}$ & $\begin{array}{l}\text { Decoction, juice } \\
\text { extract, } \\
\text { mastication, } \\
\text { poultices, soups, }\end{array}$ & 0.21 & 95 & 0.3 & 0.11 & s.s, s.w & $\begin{array}{l}\text { Alade \& Ajibesin } \\
\text { 2017, Akwaji et } \\
\text { al.2017 }\end{array}$ \\
\hline $\begin{array}{l}\text { Jatropha gossypifolia L. } \\
\text { BDU } 150\end{array}$ & Euphorbiaceae & Botuje-pupa (Y.) & $\begin{array}{l}\text { Wild } \\
\text { cassava }\end{array}$ & Malaria & Leaves & Juice extract & 0.1 & 75 & 0.05 & 0.05 & s.w & lyama \& Idu 2015 \\
\hline $\begin{array}{l}\text { Kalanchoe pinnata (Lam.) Pers. } \\
\text { BDU } 169\end{array}$ & Crassulaceae & $\begin{array}{l}\text { Oken (IJ.); Obekpokpa } \\
\text { (U.), Nkwaaka (EK.) }\end{array}$ & $\begin{array}{l}\text { Resurrectio } \\
\text { n plant }\end{array}$ & $\begin{array}{l}\text { Fever, excision of } \\
\text { umbilical cord, } \\
\text { persistent cough, Ear } \\
\text { infection, boils, } \\
\text { convulsion }\end{array}$ & $\begin{array}{l}\text { Leaves, } \\
\text { roots }\end{array}$ & $\begin{array}{l}\text { Decoction, juice } \\
\text { extract, }\end{array}$ & 0.13 & 80 & 0.3 & 0.23 & s.s & $\begin{array}{l}\text { Alade \& Ajibesin } \\
\text { 2017, Ariwaodo } \\
\text { et al. } 2012\end{array}$ \\
\hline $\begin{array}{l}\text { Khaya grandifolia C. DC. } \\
\text { BDU } 268\end{array}$ & Meliaceae & Oganwo (Y.) & Mahogany & Malaria, hemorrhoid & Stembark & $\begin{array}{l}\text { Decoction, } \\
\text { infusion }\end{array}$ & 0.1 & 80 & 0.15 & 0.15 & s.w & $\begin{array}{l}\text { lyama \& Idu } \\
\text { 2015, Ariwaodo } \\
\text { et al. } 2012\end{array}$ \\
\hline $\begin{array}{l}\text { Khaya ivoriensis A. Chev } \\
\text { BDU } 158\end{array}$ & Meliaceae & Oganwo (Y.) & $\begin{array}{l}\text { African } \\
\text { Mahogany }\end{array}$ & Malaria & Stembark & Decoction & 0.14 & 75 & 0.05 & 0.03 & s.w & lyama \& Idu 2015 \\
\hline $\begin{array}{l}\text { Kigelia africana (Lamb) Benth } \\
\text { BDU } 165\end{array}$ & Bignoniaceae & & $\begin{array}{l}\text { Sausage } \\
\text { tree }\end{array}$ & $\begin{array}{l}\text { Malaria, female } \\
\text { infertility }\end{array}$ & Roots & Infusion & 0.09 & 77 & 0.1 & 0.11 & $\begin{array}{l}\text { s.e, s.s., s.w } \\
\end{array}$ & $\begin{array}{l}\text { lyama \& Idu } \\
\text { 2015, Soladoyeet } \\
\text { al. 2014 }\end{array}$ \\
\hline $\begin{array}{l}\text { Lannea taraxalifolia A. Rich } \\
\text { BDU } 170\end{array}$ & Anacardiaceae & Yanrin (Y.) & & Dislocation & & & 0.06 & 75 & 0.05 & 0.08 & s.w & Lawal et al. 2010 \\
\hline $\begin{array}{l}\text { Lantana camara L. } \\
\text { BDU } 153\end{array}$ & Verbanaceae & Ewon-agogo (Y.) & Wild Sage & Malaria & Leaves & Decoction & 0.06 & 75 & 0.05 & 0.08 & s.w & lyama\&ldu 2015 \\
\hline $\begin{array}{l}\text { Lawsonia inermis L. } \\
\text { BDU } 177\end{array}$ & Lythraceae & Laali (Y.) & $\begin{array}{l}\text { Henna } \\
\text { plant }\end{array}$ & Malaria, skin care & Leaves & Decoction & 0.03 & 50 & 0.1 & 0.33 & s.w & Iyama \& Idu 2015 \\
\hline
\end{tabular}


Ethnobotany Research and Applications

\begin{tabular}{|c|c|c|c|c|c|c|c|c|c|c|c|c|}
\hline $\begin{array}{l}\text { Lecaniodiscuscupanoides Planch } \\
\text { exBenth } \\
\text { BDU } 161\end{array}$ & Sapindaceae & Akika (Y.) & $\begin{array}{l}\text { Lecaniodisc } \\
\text { us }\end{array}$ & $\begin{array}{l}\text { Malaria, fever, } \\
\text { purgative, typhoid, } \\
\text { jaundice, cough }\end{array}$ & $\begin{array}{l}\text { Leaves, } \\
\text { stembark, } \\
\text { seeds, roots }\end{array}$ & Decoction & 0.1 & 80 & 0.3 & 0.6 & s.W & $\begin{array}{l}\text { Ariwaodo et al. } \\
2012 \text {, lyama \& } \\
\text { Idu } 2015\end{array}$ \\
\hline $\begin{array}{l}\text { Lippia multiflora Poir } \\
\text { BDU } 141\end{array}$ & Verbanaceae & $\begin{array}{l}\text { Efinin-gorogoro, } \\
\text { Efinrin-oko (Y.) }\end{array}$ & Sweet leaf & Malaria & $\begin{array}{l}\text { Whole } \\
\text { plant }\end{array}$ & Decoction & 0.06 & 50 & 0.1 & 0.08 & s.w & lyama \& Idu 2015 \\
\hline $\begin{array}{l}\text { Lophira alata Banks ex Gaertn. f. } \\
\text { BDU } 155\end{array}$ & Ochnaceae & Pahan (Y.) & Iron wood & Malaria & Stembark & Decoction & 0.03 & 50 & 0.1 & 0.16 & s.w & lyama \& Idu 2015 \\
\hline $\begin{array}{l}\text { Ludwigia hyssopifolia (G. Don) } \\
\text { Exell } \\
\text { BDU } 144\end{array}$ & Onagraceae & Bini-sensen (B.) & $\begin{array}{l}\text { Water } \\
\text { primrose }\end{array}$ & Malaria & Leaves & Decoction & 0.06 & 75 & 0.1 & 0.08 & s.s & lyama \& Idu 2015 \\
\hline $\begin{array}{l}\text { Luffa cylindrical (L.) M.J. Roem } \\
\text { BDU } 165\end{array}$ & Cucurbitaceae & Aniamme (IK.) & $\begin{array}{l}\text { Sponge } \\
\text { guord }\end{array}$ & $\begin{array}{l}\text { Cough, laxative, } \\
\text { emetic }\end{array}$ & $\begin{array}{l}\text { Leaves, } \\
\text { stem, } \\
\text { seeds, roots }\end{array}$ & $\begin{array}{l}\text { Decoction, } \\
\text { infusion }\end{array}$ & 0.06 & 50 & 0.15 & 0.3 & s.s & $\begin{array}{l}\text { Ajibesin et al. } \\
2008 \text { \& } 2012\end{array}$ \\
\hline $\begin{array}{l}\text { Mallotus cordifolia Muell.arg } \\
\text { BDU } 134\end{array}$ & Euphorbiaceae & Ebewosa (B.) & & Malaria & Leaves & Decoction & 0.05 & 80 & 0.05 & 0.1 & s.s & lyama \& Idu 2015 \\
\hline Mangifera indica L. BDU 170 & Anarcadiaceae & $\begin{array}{l}\text { Mangoro (I.), Mankwo } \\
\text { (IK.), Imagolo (U.) }\end{array}$ & Mango & $\begin{array}{l}\text { Malaria, typhoid fever, } \\
\text { diabetes, memory } \\
\text { enhancer, headache, } \\
\text { jaundice, skin disease, } \\
\text { astringent, sore } \\
\text { throat, dysentry }\end{array}$ & $\begin{array}{l}\text { Leaves, } \\
\text { stem, bark, } \\
\text { fruits }\end{array}$ & $\begin{array}{l}\text { Baths, decoction, } \\
\text { maceration, } \\
\text { meals }\end{array}$ & 0.25 & 60 & 0.55 & 0.22 & s.e, s.s & $\begin{array}{l}\text { Alade \& Ajibesin } \\
\text { 2017, Ariwaodo } \\
\text { et al.2012, Akwaji } \\
\text { et al. 2017 }\end{array}$ \\
\hline $\begin{array}{l}\text { Manihot esculenta Crantz } \\
\text { BDU } 172\end{array}$ & Euphorbiaceae & $\begin{array}{l}\text { Akpu, jigbo, Ugboro, } \\
\text { jiaph (I.), Imidaka (U.) }\end{array}$ & Cassava & $\begin{array}{l}\text { Eye problems, wound } \\
\text { healing, chronic otitis }\end{array}$ & $\begin{array}{l}\text { Leaves, } \\
\text { juvenile } \\
\text { roots }\end{array}$ & $\begin{array}{l}\text { Leaf juice extract, } \\
\text { root decoction }\end{array}$ & 0.1 & 75 & 0.15 & 0.15 & s.e, s.s & $\begin{array}{l}\text { Alade \& Ajibesin } \\
2017\end{array}$ \\
\hline $\begin{array}{l}\text { Mansonia altissima A. Chev. } \\
\text { BDU } 55\end{array}$ & Sterucliaceae & Ofun $(Y)$. & $\begin{array}{l}\text { African } \\
\text { black } \\
\text { walnut }\end{array}$ & $\begin{array}{l}\text { Constipation, leprosy, } \\
\text { yaws, scabies, syphilis }\end{array}$ & $\begin{array}{l}\text { Twig bark, } \\
\text { stembark, } \\
\text { roots }\end{array}$ & $\begin{array}{l}\text { Decoction, } \\
\text { infusion }\end{array}$ & 0.11 & 95 & 0.3 & 0.22 & s.w & Lawal et al. 2010 \\
\hline $\begin{array}{l}\text { Milicia excelsa (Welw.) C.C. Berg } \\
\text { BDU } 183\end{array}$ & Meliaceae & Oje (I.), Iroko $(\mathrm{Y}$.$) ,$ & Iroko & $\begin{array}{l}\text { Malaria, rheumatism, } \\
\text { nausea, abdominal } \\
\text { pain, insomnia }\end{array}$ & $\begin{array}{l}\text { Stembark, } \\
\text { roots }\end{array}$ & Decoction & 0.07 & 50 & 0.3 & 0.4 & s.e, s.w & $\begin{array}{l}\text { Ariwaodo et al. } \\
\text { 2012, lyama } \& \\
\text { Idu 2015, Lawal } \\
\text { et al. 2010 }\end{array}$ \\
\hline $\begin{array}{l}\text { Microdesmis puberula Hook. f. } \\
\text { ex Planch } \\
\text { BDU } 166\end{array}$ & Euphorbiaceae & Uperi (I.), Ido-apata (Y.) & $\begin{array}{l}\text { Microdesmi } \\
\mathrm{s}\end{array}$ & Malaria & Leaves & Decoction & 0.03 & 50 & 0.05 & 0.16 & s.e, s.w & Iyama \& Idu 2015 \\
\hline $\begin{array}{l}\text { Millettia griffonianas Bail. } \\
\text { BDU } 122\end{array}$ & Fabaceae & Ito $(Y)$. & & General weakness & & & 0.07 & 50 & 0.05 & 0.06 & s.w & Lawal et al. 2010 \\
\hline $\begin{array}{l}\text { Momordica balsamina L. } \\
\text { BDU 173 }\end{array}$ & Curcubitaceae & $\begin{array}{l}\text { Sibifuka, Akbanndene } \\
\text { (I.), Ejirin (Y.) }\end{array}$ & & Haemorrhoid & $\begin{array}{l}\text { Whole } \\
\text { plant }\end{array}$ & Decoction & 0.06 & 75 & 0.05 & 0.08 & s.e, s.w & $\begin{array}{l}\text { Ajibesin et al. } \\
2008 \& 2012\end{array}$ \\
\hline $\begin{array}{l}\text { Momordica charantia L. } \\
\text { BDU } 248\end{array}$ & Curcubitaceae & $\begin{array}{l}\text { Aloose (O.), } \\
\text { Akbanndene (IK.), } \\
\text { Ejinrin-were (Y.), }\end{array}$ & $\begin{array}{l}\text { African } \\
\text { cucumber,b } \\
\text { alsam pear }\end{array}$ & $\begin{array}{l}\text { Malaria, ulcers, burns, } \\
\text { skin infections, } \\
\text { disbetes, convulsion, } \\
\text { vermifuge, } \\
\text { aphrodisiac, } \\
\text { gonorrhea, yaws, } \\
\text { boils, malignant } \\
\text { ulcers, diabetes, } \\
\text { gastrointestinal } \\
\text { problems, viral } \\
\text { diseases, female } \\
\text { infertility, malaria }\end{array}$ & Leaves & $\begin{array}{l}\text { Decoction, juice } \\
\text { extract }\end{array}$ & 0.11 & 95 & 0.4 & 0.32 & s.e, s.w & $\begin{array}{l}\text { Ajibesin et al. } \\
2008 \text { \& } \\
2012 \text {,Ariwaodo et } \\
\text { al. 2012, lyama \& } \\
\text { Idu 2015, } \\
\text { Soladoye } \text { et al. } \\
2014\end{array}$ \\
\hline $\begin{array}{l}\text { Mondia whitei (Hook. f.) Skeels } \\
\text { BDU } 186\end{array}$ & Periplocacaeae & Isirigun (Y.) & Mondi & Malaria & $\begin{array}{l}\text { Whole } \\
\text { plant, roots }\end{array}$ & Decoction & 0.06 & 50 & 0.05 & 0.08 & S.W & lyama \& Idu 2015 \\
\hline
\end{tabular}


Ethnobotany Research and Applications

\begin{tabular}{|c|c|c|c|c|c|c|c|c|c|c|c|c|}
\hline $\begin{array}{l}\text { Morinda lucida Benth } \\
\text { BDU } 305\end{array}$ & Rubiaceae & Oruwo (Y.), Njisi (I.). & $\begin{array}{l}\text { Brimstone } \\
\text { tree }\end{array}$ & $\begin{array}{l}\text { Malaria, female } \\
\text { infertility }\end{array}$ & $\begin{array}{l}\text { Leaves, } \\
\text { stembark }\end{array}$ & $\begin{array}{l}\text { Decoction, juice } \\
\text { extract }\end{array}$ & 0.11 & 50 & 0.1 & 0.09 & s.e, s.w & $\begin{array}{l}\text { lyama \& Idu } \\
\text { 2015, Soladoyeet } \\
\text { al. } 2014\end{array}$ \\
\hline $\begin{array}{l}\text { Morinda morindiodes (Barker). } \\
\text { Milne-Redh } \\
\text { BDU } 277\end{array}$ & Rubiaceae & $\begin{array}{l}\text { Poju-owiwi, Oju- } \\
\text { ologbo (Y.) }\end{array}$ & Morinda & Malaria & $\begin{array}{l}\text { Leaves, } \\
\text { stembark }\end{array}$ & Decoction & 0.05 & 90 & 0.05 & 0.1 & s.w & Iyama \& Idu 2015 \\
\hline $\begin{array}{l}\text { Moringa oleifera Lam. } \\
\text { BDU } 197\end{array}$ & Moringaceae & Ewe-igbale (Y.) & $\begin{array}{l}\text { Horse } \\
\text { radish tree }\end{array}$ & $\begin{array}{l}\text { Malaria, vitamin } \\
\text { supplement, acute } \\
\text { rheumatism }\end{array}$ & Leaves & $\begin{array}{l}\text { Decoction, } \\
\text { maceration }\end{array}$ & 0.25 & 60 & 0.2 & 0.08 & s.w & $\begin{array}{l}\text { Alade \& Ajibesin } \\
\text { 2017, Iyama \& } \\
\text { Idu 2015, Lawal } \\
\text { et al.2010 }\end{array}$ \\
\hline $\begin{array}{l}\text { Musa acuminata L. } \\
\text { BDU } 198\end{array}$ & Musaceae & & $\begin{array}{l}\text { Dwarf } \\
\text { banana }\end{array}$ & Diabetes & Fruits & & 0.03 & 50 & 0.05 & 0.16 & s.e & Akwaji et al.2017 \\
\hline $\begin{array}{l}\text { Musa paradisiaca L. } \\
\text { BDU } 107\end{array}$ & Musaceae & Mbana (I.); Ogede (Y.) & Plantain & Malaria, infertility & $\begin{array}{l}\text { Leaves, } \\
\text { stembark, } \\
\text { fruits }\end{array}$ & Decoction & 0.06 & 50 & 0.1 & 0.16 & s.e, s.w & $\begin{array}{l}\text { Alade \&s Ajibesin } \\
2017 \text {, Iyama \& } \\
\text { Idu } 2015\end{array}$ \\
\hline $\begin{array}{l}\text { Musa sapientum L. } \\
\text { BDU } 31\end{array}$ & Musaceae & Ogede-were $(Y)$. & Banana & Malaria & Leaves & Decoction & 0.05 & 70 & 0.05 & 0.1 & s.w & Lawal et al. 2010 \\
\hline $\begin{array}{l}\text { Musanga cecropioides R. Br. ex } \\
\text { Tedlie } \\
\text { BDU } 212\end{array}$ & Moraceae & Agbawo, Oro (Y.) & $\begin{array}{l}\text { Cork wood, } \\
\text { Umbrella } \\
\text { tree }\end{array}$ & $\begin{array}{l}\text { Malaria, dysentery, } \\
\text { cough, vemifuge }\end{array}$ & $\begin{array}{l}\text { Leaves, } \\
\text { stembark, } \\
\text { roots }\end{array}$ & Decoction & 0.1 & 75 & 0.2 & 0.2 & s.w & $\begin{array}{l}\text { Ariwaodo et al. } \\
2012\end{array}$ \\
\hline $\begin{array}{l}\text { Napoleona imperialis P. Beauv. } \\
\text { BDU } 240\end{array}$ & Lecythidaceae & $\begin{array}{l}\text { Nnekeloche, } \\
\text { abakalabaka (I.) }\end{array}$ & $\begin{array}{l}\text { Vogel's } \\
\text { Napoleona }\end{array}$ & $\begin{array}{l}\text { Blood clot removal in } \\
\text { freshly birthed } \\
\text { women, vermifuge, } \\
\text { gonorrhea, fevers }\end{array}$ & $\begin{array}{l}\text { Leaves, } \\
\text { stem, roots }\end{array}$ & $\begin{array}{l}\text { Decoction, } \\
\text { infusion }\end{array}$ & 0.15 & 80 & 0.2 & 0.13 & s.e & $\begin{array}{l}\text { Obute 2005\& } \\
2007\end{array}$ \\
\hline $\begin{array}{l}\text { Nasturtium officinale R. Br } \\
\text { BDU } 401\end{array}$ & Brassicaceae & Aguba (IK.) & Watercress & Impotence & Leaves & Decoction & 0.02 & 50 & 0.05 & 0.3 & s.s & $\begin{array}{l}\text { Ajibesinet al. } \\
2008\end{array}$ \\
\hline $\begin{array}{l}\text { Nauclea diderrichï (De Wild) } \\
\text { Merr } \\
\text { BDU } 408\end{array}$ & Rubiaceae & Ope (U.), Opepe (Y.) & $\begin{array}{l}\text { African } \\
\text { peach }\end{array}$ & Malaria & Stembark & Decoction & 0.06 & 50 & 0.05 & 0.08 & s.s, s.w & lyama \& Idu 2015 \\
\hline $\begin{array}{l}\text { Nauclea latifolia (Smith) Bruce } \\
\text { BDU } 452\end{array}$ & Rubiaceae & Egbesi (Y.) & $\begin{array}{l}\text { African } \\
\text { peach }\end{array}$ & $\begin{array}{l}\text { Malaria, fevers, } \\
\text { blennorrhea, colic, } \\
\text { constipation, } \\
\text { jaundice, otitis, } \\
\text { dysmenorrheal }\end{array}$ & $\begin{array}{l}\text { Leaves, } \\
\text { stembark, } \\
\text { roots }\end{array}$ & $\begin{array}{l}\text { Decoction, } \\
\text { tincture }\end{array}$ & 0.21 & 95 & 0.4 & 0.19 & s.s, s.w & $\begin{array}{l}\text { Akwaji et al.2017, } \\
\text { lyama \& Idu } 2015\end{array}$ \\
\hline $\begin{array}{l}\text { Newbouldia laevis (Beauv.) } \\
\text { Seeman ex Bureau } \\
\text { BDU } 399\end{array}$ & Bignoniaceae & $\begin{array}{l}\text { Ogirisi (O.), } \\
\text { Oke-ogirishi (I.), Akoko } \\
\text { (Y.). }\end{array}$ & $\begin{array}{l}\text { Smooth } \\
\text { Newbouldi } \\
\text { a, Tree of } \\
\text { life, Fertility } \\
\text { tree }\end{array}$ & $\begin{array}{l}\text { Eye problems. } \\
\text { childbirth, } \\
\text { constipation, malaria, } \\
\text { septic wounds, } \\
\text { convulsion, epilepsy, } \\
\text { bleeding, migraine, } \\
\text { eye infection, skin } \\
\text { disease, infertility }\end{array}$ & $\begin{array}{l}\text { Leaves, } \\
\text { stembark, } \\
\text { roots }\end{array}$ & $\begin{array}{l}\text { Decoction, } \\
\text { infusion, topical } \\
\text { application of } \\
\text { leaf juice, } \\
\text { poultice }\end{array}$ & 0.19 & 71 & 0.6 & 0.28 & s.e, s.s, s.w & $\begin{array}{l}\text { Ariwaodo et al. } \\
\text { 2012, lyama \& } \\
\text { Idu 2015, Lawal } \\
\text { et al. } 2010\end{array}$ \\
\hline $\begin{array}{l}\text { Ocimum gratissimum L. } \\
\text { BDU } 470\end{array}$ & Lamiaceae & $\begin{array}{l}\text { Nchuanwu (I.), Eran, } \\
\text { Ufuo-oyibo (U.), } \\
\text { Efinrin-ajase (Y.). }\end{array}$ & $\begin{array}{l}\text { Tea bush, } \\
\text { Scent leaf }\end{array}$ & $\begin{array}{l}\text { Constipation, } \\
\text { Diabetes Miletus, } \\
\text { vermifuge, malaria }\end{array}$ & $\begin{array}{l}\text { Leaves, leaf } \\
\text { extract } \\
+ \text { Viscum } \\
\text { album }\end{array}$ & $\begin{array}{l}\text { Decoction, } \\
\text { infusion }\end{array}$ & 0.25 & 60 & 0.2 & 0.08 & s.e, s.s, s.w & $\begin{array}{l}\text { lyama \& Idu } \\
\text { 2015, Alade \& } \\
\text { Ajibesin } 2017\end{array}$ \\
\hline $\begin{array}{l}\text { Palisota hirsuta (Thumb.)K. } \\
\text { Schum. BDU } 350\end{array}$ & Commelinaceae & $\begin{array}{l}\text { Ikpereaturu(I.), Asatie } \\
\text { (EK.) }\end{array}$ & Palisota & $\begin{array}{l}\text { Rheumatism, arthritis, } \\
\text { malaria, boils, } \\
\text { gonorrhea }\end{array}$ & $\begin{array}{l}\text { Leaves, } \\
\text { stem }\end{array}$ & $\begin{array}{l}\text { Decoction, } \\
\text { infusion, poultice }\end{array}$ & 0.09 & 77 & 0.3 & 0.14 & s.e,s.S. & Iyama \& Idu 2015 \\
\hline $\begin{array}{l}\text { Parinari macrophylla Sabine } \\
\text { BDU } 352\end{array}$ & Rosaceae & Abere (Y.) & $\begin{array}{l}\text { Neouoil } \\
\text { tree }\end{array}$ & Malaria & Seeds & Decoction & 0.06 & 50 & 0.05 & 0.08 & s.w & Iyama\&ldu 2015 \\
\hline $\begin{array}{l}\text { Parkia bicolor A. Chev. BDU } \\
125\end{array}$ & Fabaceae & Iru (Y.) & & Diarrhea, dysentery & & & 0.07 & 85 & 0.1 & 0.14 & s.e & Lawal et al. 2010 \\
\hline
\end{tabular}


Ethnobotany Research and Applications

\begin{tabular}{|c|c|c|c|c|c|c|c|c|c|c|c|c|}
\hline $\begin{array}{l}\text { Parquetinanigrescens(Afzel) } \\
\text { Bullock BDU } 246\end{array}$ & $\begin{array}{l}\text { Periplocaceae } \\
\end{array}$ & Ewe-ogbo (Y.) & $\begin{array}{l}\text { African } \\
\text { parquetina }\end{array}$ & Malaria & Leaves & $\begin{array}{l}\text { Decoction, } \\
\text { infusion }\end{array}$ & 0.06 & 75 & 0.05 & 0.08 & s.w & lyama \& Idu 2015 \\
\hline $\begin{array}{l}\text { Pastinaca sativa L. } \\
\text { BDU } 313\end{array}$ & Apiaceae & Udeghe (K.) & Parsnip & Cough, hypertension & Leaves & Decoction & 0.06 & 50 & 0.1 & 0.16 & s.s & $\begin{array}{l}\text { Ajibesin } e t \text { al. } \\
2008 \& 2012\end{array}$ \\
\hline $\begin{array}{l}\text { Pentaclethra macrophylla Benth. } \\
\text { BDU } 227\end{array}$ & Fabaceae & Ugba (I.) & $\begin{array}{l}\text { African oil } \\
\text { bean tree }\end{array}$ & $\begin{array}{l}\text { Stuttering, skin } \\
\text { diseases, convulsion, } \\
\text { gonorrhea, infertility, } \\
\text { wound, itching }\end{array}$ & $\begin{array}{l}\text { Leaves, } \\
\text { pod, seeds, } \\
\text { stembark }\end{array}$ & $\begin{array}{l}\text { Poultice of oil } \\
\text { extract }\end{array}$ & 0.09 & 72 & 0.4 & 0.44 & s.e & Akwaji et al. 2017 \\
\hline $\begin{array}{l}\text { Peperomia pellucida (L.) H.B. \& } \\
\text { K. } \\
\text { BDU } 318\end{array}$ & $\begin{array}{l}\text { Piperaceae } \\
\text {. }\end{array}$ & Rinrin (Y.) & Silver Bush & Malaria & Leaves & Decoction & 0.11 & 86 & 0.1 & 0.4 & s.w & lyama \& Idu 2015 \\
\hline $\begin{array}{l}\text { Pergularia daemia (Forsk.) Choiv } \\
\text { BDU } 341\end{array}$ & Asclepiadaceae & $\begin{array}{l}\text { Utaezi (I.), Jagborokun, } \\
\text { rogbo-aguntan (Y.) }\end{array}$ & Pergularia & $\begin{array}{l}\text { Malaria, boils, } \\
\text { typhoid, cough }\end{array}$ & Leaves & $\begin{array}{l}\text { Decoction of } \\
\text { leaves + Carica } \\
\text { papaya, Vernonia } \\
\text { amygdalina, } \\
\text { Ananas comosus } \\
\text { and } \\
\text { Chromolaena } \\
\text { odoratajuice, } \\
\text { infusion, } \\
\text { maceration, } \\
\text { poultice }\end{array}$ & 0.21 & 95 & 0.2 & 0.10 & s.e, s.w & lyama \& Idu 2015 \\
\hline $\begin{array}{l}\text { Persea americana Mill } \quad \text { BDU } \\
211\end{array}$ & Lauraceae & $\begin{array}{l}\text { Ube-oyibo (I.), Pia (Y.), } \\
\text { Ur-uvwon (U.) }\end{array}$ & $\begin{array}{l}\text { Avocado } \\
\text { pear }\end{array}$ & $\begin{array}{l}\text { Malaria, typhoid, } \\
\text { diabetes, high blood } \\
\text { pressure }\end{array}$ & $\begin{array}{l}\text { Leaves, } \\
\text { stembark, } \\
\text { seeds }\end{array}$ & $\begin{array}{l}\text { Decoction, juice } \\
\text { extract }\end{array}$ & 0.17 & 98 & 0.2 & 0.11 & s.e, s.s, s.w & $\begin{array}{l}\text { lyama \& Idu } \\
2015 \text {, Akwajiet al. } \\
2017\end{array}$ \\
\hline Petivera alliacea L. BDU 206 & $\begin{array}{l}\text { Phytolaccaceae } \\
\end{array}$ & Awogba (Y.) & $\begin{array}{l}\text { Skunk } \\
\text { weed, } \\
\text { Gully-root }\end{array}$ & Malaria & Leaves & Decoction & 0.02 & 50 & 0.1 & 0.3 & s.w & lyama \& Idu 2015 \\
\hline $\begin{array}{l}\text { Phyllanthus amarus Schum et } \\
\text { Thonn BDU } 215\end{array}$ & Euphorbiaceae & Eyin-olobe (Y.) & $\begin{array}{l}\text { Small leaf, } \\
\text { stone } \\
\text { breaker }\end{array}$ & Malaria & Leaves & $\begin{array}{l}\text { Decoction, } \\
\text { infusion }\end{array}$ & 0.02 & 75 & 0.1 & 0.3 & s.w & Iyama \& Idu 2015 \\
\hline $\begin{array}{l}\text { Phyllanthus muellerianus (O. } \\
\text { Ktz) Exell } \\
\text { BDU } 239\end{array}$ & Euphorbiacea & Egun-eja (Y.) & Myrobalan & Malaria & Leaves & Maceration & 0.09 & 50 & 0.1 & 0.06 & s.w & lyama \& Idu 2015 \\
\hline $\begin{array}{l}\text { Physallis angulata L. } \\
\text { BDU } 315\end{array}$ & Solanaceae & Koropo (Y.) & $\begin{array}{l}\text { Ground } \\
\text { angular } \\
\text { cherry }\end{array}$ & Malaria & Leaves & Decoction & 0.07 & 50 & 0.1 & 0.07 & s.w & lyama \& Idu 2015 \\
\hline $\begin{array}{l}\text { Picralima nitida (Stapf) Th. \& H. } \\
\text { Dur } \\
\text { BDU } 200\end{array}$ & Apocynaceae & Erin, Eso-abere (Y.) & Picralima & Malaria & Seeds & Decoction & 0.06 & 50 & 0.1 & 0.08 & s.w & $\begin{array}{l}\text { lyama \& Idu } \\
2015\end{array}$ \\
\hline $\begin{array}{l}\text { Piper guineense Schum. \& } \\
\text { Thonn } \\
\text { BDU } 279\end{array}$ & Piperaceae & $\begin{array}{l}\text { Iyere (Y.), Ebe-ahanbi } \\
\text { (B.) }\end{array}$ & $\begin{array}{l}\text { Climbing } \\
\text { black } \\
\text { pepper }\end{array}$ & $\begin{array}{l}\text { Malaria, female } \\
\text { infertility, newly } \\
\text { birthed women, } \\
\text { stomachache }\end{array}$ & 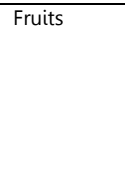 & Decoction & 0.18 & 80 & 0.2 & 0.11 & s.w & $\begin{array}{l}\text { Akwaji et al. } \\
\text { 2017, Iyama \& } \\
\text { Idu 2015, } \\
\text { Soladoyeet al. } \\
\text { 2014, Ugbogu \& } \\
\text { Chukwuma } 2019\end{array}$ \\
\hline $\begin{array}{l}\text { Piper nigrum Schum. \& Thonn. } \\
\text { BDU } 191\end{array}$ & Piperaceae & Uziza (I.) & & $\begin{array}{l}\text { After birth womb } \\
\text { stabilizer treatment in } \\
\text { women }\end{array}$ & $\begin{array}{l}\text { Leaves and } \\
\text { seeds }\end{array}$ & $\begin{array}{l}\text { Decoction, } \\
\text { tinctures }\end{array}$ & 0.17 & 88 & 0.1 & 0.02 & s.e & $\begin{array}{l}\text { Obute } 2005 \& \\
2007\end{array}$ \\
\hline $\begin{array}{l}\text { Prosopis africanaGuill\&Perr } \\
\text { BDU } 113\end{array}$ & Fabaceae & Ayan (Y.) & & Pile & & & 0.13 & 50 & 0.1 & 0.04 & s.w & Lawal et al. 2010 \\
\hline $\begin{array}{l}\text { Prunus domestica L. } \\
\text { BDU } 108\end{array}$ & Rosaceae & Pulomu (1.) & Plum & Eczema & Leaf ash & $\begin{array}{l}\text { Poultice, topical } \\
\text { application }\end{array}$ & 0.06 & 75 & 0.1 & 0.08 & s.e & Ajuru 2018 \\
\hline
\end{tabular}


Ethnobotany Research and Applications

\begin{tabular}{|c|c|c|c|c|c|c|c|c|c|c|c|c|}
\hline $\begin{array}{l}\text { Psidium guajava L. } \\
\text { BDU } 255\end{array}$ & Myrtaceae & $\begin{array}{l}\text { Gova (I.), Igobe (U.); } \\
\text { Gilofa (Y.) }\end{array}$ & Guava & $\begin{array}{l}\text { Anaemia, malaria, } \\
\text { diarrhea, dysentery, } \\
\text { spews, stools, fever, } \\
\text { pain, female infertility }\end{array}$ & $\begin{array}{l}\text { Leaves, } \\
\text { bark, root }\end{array}$ & $\begin{array}{l}\text { Decoction, } \\
\text { maceration }\end{array}$ & 0.23 & 65 & 0.5 & 0.19 & s.e, s.s, s.w & $\begin{array}{l}\text { Alade \& Ajibesin } \\
2017 \text {, lyama } \\
\& \\
\text { Idu } \\
2015 \text {, Soladoye } s \text {. } \\
2014 \text {, Lawal et al. } \\
2010\end{array}$ \\
\hline $\begin{array}{l}\text { Pterocarpus osun Craib } \\
\text { BDU } 152\end{array}$ & Fabaceae & Osun (Y.) & Camwood & $\begin{array}{l}\text { Umbilical cord } \\
\text { antiseptic, } \\
\text { rheumatism, eczema, } \\
\text { gonorrhea, } \\
\text { candidasis, acne, } \\
\text { amenorrhea }\end{array}$ & $\begin{array}{l}\text { Leaves, } \\
\text { stem, } \\
\text { stembark, } \\
\text { heartwood, } \\
\text { roots }\end{array}$ & $\begin{array}{l}\text { Baths, poultice, } \\
\text { powdered }\end{array}$ & 0.22 & 90 & 0.4 & 0.15 & s.w & $\begin{array}{l}\text { Akwaji et al. } \\
2017 \text {,Lawal et al. } \\
2010\end{array}$ \\
\hline $\begin{array}{l}\text { Pterocarpus santalinoides D.C } \\
\text { BDU } 266\end{array}$ & Fabaceae & $\begin{array}{l}\text { Nturukpa (I.), } \\
\text { Gbengben (Y.), }\end{array}$ & $\begin{array}{l}\text { Winged } \\
\text { fruit }\end{array}$ & Malaria, anti-ageing & $\begin{array}{l}\text { Leaves, } \\
\text { stem }\end{array}$ & Decoction & 0.09 & 72 & 0.1 & 0.11 & 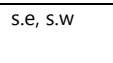 & lyama \& Idu 2015 \\
\hline $\begin{array}{l}\text { Pycanthus angolensis (Welw) } \\
\text { Warb. } \\
\text { BDU } 174\end{array}$ & Myristicaceae & Akomu (Y.) & $\begin{array}{l}\text { African } \\
\text { nutmeg }\end{array}$ & $\begin{array}{l}\text { Malaria, purgative, } \\
\text { enema, skin disease }\end{array}$ & $\begin{array}{l}\text { Leaves, } \\
\text { stembark }\end{array}$ & Decoction & 0.05 & 60 & 0.2 & 0.4 & s.w & $\begin{array}{l}\text { Akwaji et al } \\
.2017, \text { lyama \& } \\
\text { Idu } 2015 \\
\end{array}$ \\
\hline $\begin{array}{l}\text { Pyrenacantha staudtii Engl } \\
\text { BDU } 341\end{array}$ & Icacinaceae & $\begin{array}{l}\text { Arorodegbo, Oroto- } \\
\text { agba (Y.) }\end{array}$ & $\begin{array}{l}\text { Pyrenacant } \\
\text { ha }\end{array}$ & Malaria & Leaves & Juice extract & 0.06 & 50 & 0.05 & 0.08 & s.w & Iyama \& Idu 2015 \\
\hline $\begin{array}{l}\text { Ricinus communis L. } \\
\text { BDU } 386\end{array}$ & Euphorbiaceae & $\begin{array}{l}\text { Ogiriaro (O.), Ecastor } \\
\text { (U.), Laa (Y.) }\end{array}$ & $\begin{array}{l}\text { Castor } \\
\text { bean }\end{array}$ & $\begin{array}{l}\text { As purgative, } \\
\text { stomachaches, as } \\
\text { vermifuge } \\
\text { skin disease }\end{array}$ & $\begin{array}{l}\text { Leaves, } \\
\text { seeds }\end{array}$ & $\begin{array}{l}\text { Decoction, juice, } \\
\text { syrup, topical } \\
\text { application }\end{array}$ & 0.27 & 80 & 0.2 & 0.07 & s.s, s.w & $\begin{array}{l}\text { Soladoyeet al } \\
2014\end{array}$ \\
\hline $\begin{array}{l}\text { Ruawolfia vomitoria Afzel } \\
\text { BDU } 262\end{array}$ & Apocynaceae & $\begin{array}{l}\text { Asofeyeje (Y.), } \\
\text { Donuinya (K.) }\end{array}$ & $\begin{array}{l}\text { Serpent } \\
\text { wood, } \\
\text { Swizzle } \\
\text { stick }\end{array}$ & $\begin{array}{l}\text { Malaria, skin disease, } \\
\text { small pox, } \\
\text { stomachache, } \\
\text { gonorrhea, waist pain, } \\
\text { urogenital tract } \\
\text { infection, hemorrhoid }\end{array}$ & $\begin{array}{l}\text { Leaves, } \\
\text { roots }\end{array}$ & $\begin{array}{l}\text { Decoction, } \\
\text { infusion }\end{array}$ & 0.18 & 80 & 0.4 & 0.22 & s.s, s.w & $\begin{array}{l}\text { Akwajiet al. 2017, } \\
\text { Iyama \& Idu } 2015\end{array}$ \\
\hline $\begin{array}{l}\begin{array}{l}\text { Rytigynia nigerica (S. Moore). } \\
\text { Robyns } \\
\text { BDU } 188\end{array} \\
\end{array}$ & Rubiaceae & Elegun-oko (Y.) & Rytigynia & Malaria & $\begin{array}{l}\text { Stembark, } \\
\text { roots }\end{array}$ & Decoction & 0.08 & 75 & 0.05 & 0.06 & s.w & Iyama \& Idu 2015 \\
\hline $\begin{array}{l}\text { Sansevieria trifasciata Prain } \\
\text { BDU } 209\end{array}$ & Agavaceae & Angolo (IK.) & $\begin{array}{l}\text { Mother-in- } \\
\text { Law's } \\
\text { tongue }\end{array}$ & Eye problem & Leaves & Infusion, tincture & 0.03 & 50 & 0.05 & 0.16 & s.s & Ajuru 2018 \\
\hline $\begin{array}{l}\text { Securidaca longipedunculata } \\
\text { Frer } \\
\text { BDU } 300\end{array}$ & $\begin{array}{l}\text { Polygalaceae } \\
\end{array}$ & Ofodo, Ipeta (Y.) & Violet tree & Malaria & Roots & Powdered & 0.07 & 85 & 0.05 & 0.07 & s.w & lyama \& Idu 2015 \\
\hline $\begin{array}{l}\text { Senna alata (L.) Roxb. } \\
\text { BDU } 377\end{array}$ & Fabaceae & $\begin{array}{l}\text { Sokien-inyanya (K.), } \\
\text { Nduruchi } \\
\text { (IK.) }\end{array}$ & $\begin{array}{l}\text { Candle } \\
\text { bush, } \\
\text { ringworm } \\
\text { bush }\end{array}$ & $\begin{array}{l}\text { Eczema, ringworm, } \\
\text { abscess, } \\
\text { inflammation, skin } \\
\text { disease, bleeding, } \\
\text { dysentery, female } \\
\text { infertility, vermifuge }\end{array}$ & $\begin{array}{l}\text { Leaves, } \\
\text { roots }\end{array}$ & $\begin{array}{l}\text { Infusion, juice } \\
\text { extract poultice }\end{array}$ & 0.13 & 50 & 0.4 & 0.3 & s.s & $\begin{array}{l}\text { Alade \& Ajibesin } \\
\text { 2017, Ariwaodo } \\
\text { et al. 2012, } \\
\text { Soladoye et al. } \\
2014\end{array}$ \\
\hline $\begin{array}{l}\text { Senna fistula L. } \\
\text { BDU } 358\end{array}$ & Fabaceae & Aidan-toro (Y.) & $\begin{array}{l}\text { Pudding } \\
\text { stick, } \\
\text { Golden } \\
\text { Shower, }\end{array}$ & Malaria & Roots & Decoction & 0.05 & 70 & 0.05 & 0.1 & s.w & lyama \& Idu 2015 \\
\hline $\begin{array}{l}\text { Senna hirsuta (L.) Irwin \& } \\
\text { Barneby } \\
\text { BDU } 340\end{array}$ & Fabaceae & Oziza (I.) & $\begin{array}{l}\text { Shower } \\
\text { tree }\end{array}$ & $\begin{array}{l}\text { Eye ache, ear ache, } \\
\text { antimicrobial, skin } \\
\text { infection, purgative }\end{array}$ & Leaves & $\begin{array}{l}\text { Juice extract, } \\
\text { poultice }\end{array}$ & 0.06 & 75 & 0.3 & 0.41 & s.e & $\begin{array}{l}\text { Ariwaodo et al. } \\
2012\end{array}$ \\
\hline
\end{tabular}


Ethnobotany Research and Applications

\begin{tabular}{|c|c|c|c|c|c|c|c|c|c|c|c|c|}
\hline $\begin{array}{l}\text { Senna occidentalis (L.) Link. } \\
\text { BDU } 101\end{array}$ & Fabaceae & $\begin{array}{l}\text { Akidiagbara (I.), Ewe } \\
\text { (Y.) }\end{array}$ & $\begin{array}{l}\text { Negro } \\
\text { coffee, } \\
\text { coffee } \\
\text { senna }\end{array}$ & $\begin{array}{l}\text { Abscess, } \\
\text { inflammation, skin } \\
\text { disease, bleeding }\end{array}$ & $\begin{array}{l}\text { Leaves, } \\
\text { roots }\end{array}$ & $\begin{array}{l}\text { Infusion, } \\
\text { poultices, }\end{array}$ & 0.08 & 88 & 0.2 & 0.4 & s.s & Aja et al. 2017 \\
\hline $\begin{array}{l}\text { Senna podocarpa Guill. \& Perr. } \\
\text { BDU } 266\end{array}$ & Fabaceae & Asunwonibile (Y.) & Senna & malaria & $\begin{array}{l}\text { Leaves, } \\
\text { stembark }\end{array}$ & Decoction & 0.04 & 75 & 0.05 & 0.13 & s.w & Iyama \& Idu 2015 \\
\hline Senna siamea Lam. BDU 261 & Fabaceae & Kasia & & Malaria, laxatives & $\begin{array}{l}\text { Leaves, } \\
\text { stembark }\end{array}$ & Decoction & 0.07 & 50 & 0.1 & 0.14 & s.e, s.s, s.w & $\begin{array}{l}\text { lyama \& Idu } \\
2015 \text {, Lawal et al. } \\
2010\end{array}$ \\
\hline $\begin{array}{l}\text { Scoparia dulcis L. } \\
\text { BDU } 318\end{array}$ & Plantaginaceae & Anyimdede (O-M.) & $\begin{array}{l}\text { Sweet- } \\
\text { broom, } \\
\text { licorice } \\
\text { weed }\end{array}$ & $\begin{array}{l}\text { Cough, antisickling, } \\
\text { memory enhancer }\end{array}$ & Leaves & Juice extract & 0.11 & 95 & 0.15 & 0.13 & s.s & $\begin{array}{l}\text { Babawale et al. } \\
\text { 2016, Abere et al. } \\
1993\end{array}$ \\
\hline $\begin{array}{l}\text { Sida acuta Burm. f. } \\
\text { BDU } 269\end{array}$ & Malvaceae & Udo, Nsiinyinya(I.) & $\begin{array}{l}\text { Broom } \\
\text { weed }\end{array}$ & Malaria & Stem & Decoction & 0.09 & 77 & 0.05 & 0.06 & s.e & Iyama \& Idu 2015 \\
\hline $\begin{array}{l}\text { Solanum lycopersicumL. } \\
\text { BDU } 134\end{array}$ & Solanaceae & Tomatos (I.) & $\begin{array}{l}\text { Tomatoes, } v \\
\text { ine berry }\end{array}$ & Ear ache & Leaves, fruit & $\begin{array}{l}\text { Juice extract } \\
\text { poultice }\end{array}$ & 0.06 & 50 & 0.1 & 0.16 & s.e, s.w & Afolayan 2020 \\
\hline $\begin{array}{l}\text { Solanum nigrum } \mathrm{L} . \\
\text { BDU } 220\end{array}$ & Solanaceae & Ebe-ape (U.), Anara (O.) & $\begin{array}{l}\text { Black/ } \\
\text { Common } \\
\text { Nightshade }\end{array}$ & $\begin{array}{l}\text { Malaria, eye infection, } \\
\text { jaundice, convulsion, }\end{array}$ & $\begin{array}{l}\text { Whole } \\
\text { plants, } \\
\text { leaves }\end{array}$ & Decoction & 0.14 & 75 & 0.2 & 0.14 & s.s & $\begin{array}{l}\text { Ajibesin et al. } \\
20082012 \text {, lyama } \\
\text { \& Idu 2015 }\end{array}$ \\
\hline $\begin{array}{l}\text { Solenostemon monostachycus } \\
\text { (P. Beauv.) Briq. } \\
\text { BDU } 391\end{array}$ & Lamiaceae & Egba (IK.) & & Measles, malaria & Leaves & Decoction & 0.07 & 50 & 0.1 & 0.14 & s.s & $\begin{array}{l}\text { Ajibesin et al. } \\
2008 \& 2012\end{array}$ \\
\hline $\begin{array}{l}\text { Sorghum bicolor (L.) Moench } \\
\text { BDU } 376\end{array}$ & Poaceae & Poroporo-okababa (Y.) & $\begin{array}{l}\text { Guinea } \\
\text { corn }\end{array}$ & $\begin{array}{l}\text { Malaria, female } \\
\text { infertility }\end{array}$ & Leaves & Decoction & 0.22 & 90 & 0.05 & 0.02 & s.w & $\begin{array}{l}\text { lyama \& Idu } \\
\text { 2015, Soladoyeet } \\
\text { al. } 2014\end{array}$ \\
\hline $\begin{array}{l}\text { Spathodea campanulata P. } \\
\text { Beauv } \\
\text { BDU } 169\end{array}$ & Bignoniaceae & Oruru, mojutoro (Y.) & $\begin{array}{l}\text { African } \\
\text { tulip, } \\
\text { Scarlett } \\
\text { bells }\end{array}$ & Malaria & Stembark & Decoction & 0.03 & 50 & 0.05 & 0.16 & s.w & Iyama \& Idu 2015 \\
\hline $\begin{array}{l}\text { Sphenocentrum jollyanum Pierre } \\
\text { BDU } 229\end{array}$ & Menispermaceae & Akerejupon (Y.) & $\begin{array}{l}\text { Sphenocen } \\
\text { trum }\end{array}$ & $\begin{array}{l}\text { Malaria, female } \\
\text { infertility }\end{array}$ & Roots & Decoction & 0.03 & 50 & 0.1 & 0.33 & s.w & $\begin{array}{l}\text { lyama \& Idu } \\
\text { 2015, Soladoyeet } \\
\text { al. } 2014\end{array}$ \\
\hline $\begin{array}{l}\text { Spondias mombin L. } \\
\text { BDU } 347\end{array}$ & Anacardiaceae & lyeye (Y.), Aginiran (O.) & Hogplum & $\begin{array}{l}\text { Malaria, gonorrhea, } \\
\text { dysentery, toothache, } \\
\text { diabetes, diuretic, } \\
\text { female infertility, cold }\end{array}$ & $\begin{array}{l}\text { Leaves, } \\
\text { stembark }\end{array}$ & Decoction & 0.14 & 71 & 0.5 & 0.32 & s.s, s.w & $\begin{array}{l}\text { Ariwaodo et al. } \\
\text { 2012, Iyama \& } \\
\text { Idu 2015, } \\
\text { Soladoyeet al. } \\
2014\end{array}$ \\
\hline $\begin{array}{l}\text { Stachytarpheta cayennensis (LC. } \\
\text { Rich) Schua } \\
\text { BDU } 194\end{array}$ & Verbanaceae & $\begin{array}{l}\text { Ebe (U.), Obibo (Y.), } \\
\text { Mbeku (K.) }\end{array}$ & $\begin{array}{l}\text { Rats's rail } \\
\text { Vervaine, } \\
\text { Blue } \\
\text { Snakeweed }\end{array}$ & Malaria & Leaves & Decoction & 0.05 & 60 & 0.1 & 0.2 & s.s, s.w & $\begin{array}{l}\text { Ajibesin } \text { et al. } \\
2008 \& \text { 2012, } \\
\text { lyama \& Idu } \\
2015, \text { Lawal et al. } \\
2010\end{array}$ \\
\hline $\begin{array}{l}\text { Stachytarpheta indica (L.) Vahl } \\
\text { BDU } 181\end{array}$ & Verbanaceae & Otuidun (O-M.) & $\begin{array}{l}\text { Rooter } \\
\text { comb }\end{array}$ & Fever, malaria & Leaves & Juice extract & 0.16 & 90 & 0.1 & 0.06 & s.s, s.W & $\begin{array}{l}\text { Ajibesin et al. } \\
2008 \text { 2012, Iyama } \\
\text { \& Idu 2015 }\end{array}$ \\
\hline $\begin{array}{l}\text { Sterculia setigera Dell. } \\
\text { BDU } 139\end{array}$ & Sterculiaceae & Ose-aware, kukuki (Y.) & & Constipation & & & 0.12 & 50 & 0.05 & 0.04 & s.w & Lawal et al. 2010 \\
\hline $\begin{array}{l}\text { Stellaria media (L.) Vill. } \\
\text { BDU250 }\end{array}$ & Caryophyllaceae & Ahiliaokuko (IK.) & Chickweed & $\begin{array}{l}\text { Cough, debility, } \\
\text { asthma }\end{array}$ & Leaves & Decoction & 0.06 & 83 & 0.15 & 0.3 & s.s & $\begin{array}{l}\text { Ajibesin et al. } \\
2008 \& 2012\end{array}$ \\
\hline $\begin{array}{l}\text { Syndrella nodiflora Gaertn } \\
\text { BDU133 }\end{array}$ & Asteraceae & Aluganbi (Y.). & Synedrella & $\begin{array}{l}\text { Malaria, sores, skin } \\
\text { infection }\end{array}$ & Leaves & Decoction & 0.1 & 90 & 0.15 & 0.15 & s.w & $\begin{array}{l}\text { Ariwaodo et al } \\
2012, \text { lyama \& } \\
\text { Idu } 2015\end{array}$ \\
\hline
\end{tabular}


Ethnobotany Research and Applications

\begin{tabular}{|c|c|c|c|c|c|c|c|c|c|c|c|c|}
\hline $\begin{array}{l}\text { Talinum fruticosum (L.) Juss. } \\
\text { BDU } 79\end{array}$ & Portulaceceae & Segi-beleke & $\begin{array}{l}\text { Waterleaf, } \\
\text { Ceylon } \\
\text { spinach }\end{array}$ & Boils, measles & Leaves & $\begin{array}{l}\text { Juice extract, } \\
\text { decoction }\end{array}$ & 0.15 & 93 & 0.1 & 0.06 & s.s & Akwaji et al. 2017 \\
\hline $\begin{array}{l}\text { Telfairia occidentalis Hook. f. } \\
\text { BDU } 170\end{array}$ & Cucurbitaceae & Ugu (I.) & $\begin{array}{l}\text { Fluted } \\
\text { pumpkin }\end{array}$ & $\begin{array}{l}\text { Dizziness, blood } \\
\text { detox, anaemia. acne, } \\
\text { headaches, blood } \\
\text { tonic, female } \\
\text { infertility, convulsion }\end{array}$ & $\begin{array}{l}\text { Leaves, } \\
\text { fruits }\end{array}$ & $\begin{array}{l}\text { Juice extract, } \\
\text { poultice }\end{array}$ & 0.3 & 90 & 0.4 & 0.13 & s.e & $\begin{array}{l}\text { Ariwaodo et al. } \\
\text { 2012, Soladoyeet } \\
\text { al. } 2014\end{array}$ \\
\hline $\begin{array}{l}\text { Terminalia avicennioides Guill. \& } \\
\text { Perr. } \\
\text { BDU } 319\end{array}$ & Combretaceae & Idi (Y.) & Baushe & $\begin{array}{l}\text { Malaria, female } \\
\text { infertility }\end{array}$ & $\begin{array}{l}\text { Leaves, } \\
\text { stembark }\end{array}$ & Decoction & 0.1 & 90 & 0.1 & 0.10 & s.w & $\begin{array}{l}\text { lyama \& Idu } \\
\text { 2015, Soladoyeet } \\
\text { al. } 2014\end{array}$ \\
\hline Terminalia catappa L. BDU 320 & Combretaceae & $\begin{array}{l}\text { Furuntu (Y.), Ebelebo } \\
\text { (E.) }\end{array}$ & $\begin{array}{l}\text { Indian } \\
\text { almond }\end{array}$ & Malaria & Leaves & Decoction & 0.06 & 50 & 0.05 & 0.08 & s.s, s.w & lyama \& Idu 2015 \\
\hline $\begin{array}{l}\text { Terminalia ivoriensis A. Chev } \\
\text { BDU } 324\end{array}$ & Combretaceae & Afara-dudu (Y.) & Black afara & Malaria, tranquilizer & $\begin{array}{l}\text { Leaves, } \\
\text { stembark }\end{array}$ & Decoction & 0.09 & 50 & 0.1 & 0.16 & s.w & $\begin{array}{l}\text { lyama \& Idu } \\
2015 \text {, Lawal et al. } \\
2010\end{array}$ \\
\hline $\begin{array}{l}\text { Terminalia superba Engl. \& Diels } \\
\text { BDU } 308\end{array}$ & Combretaceae & Egae (O.), Afara (Y.) & $\begin{array}{l}\text { Shingle } \\
\text { wood, } \\
\text { yellow pine }\end{array}$ & $\begin{array}{l}\text { Skin diseases, eye } \\
\text { infection, diarrhea, } \\
\text { dysentery, malaria, } \\
\text { hepatitis, yellow fever }\end{array}$ & $\begin{array}{l}\text { Leaves, } \\
\text { stem, roots }\end{array}$ & $\begin{array}{l}\text { Decoction, baths } \\
\text { and remedies }\end{array}$ & 0.14 & 96 & 0.4 & 0.3 & s.S, s.w & Akwaji et al. 2017 \\
\hline $\begin{array}{l}\text { Tetracarpidium conophorum } \\
\text { Hutch. \& Dalz. } \\
\text { BDU } 376\end{array}$ & Euphorbiaceae & $\begin{array}{l}\text { Ukpa (IK.), } \\
\text { Okeokpokirinya (I.), } \\
\text { Awusa (Y.), Okhue (B.) }\end{array}$ & $\begin{array}{l}\text { Nigerian } \\
\text { walnut, } \\
\text { conophor } \\
\text { tree }\end{array}$ & $\begin{array}{l}\text { Haemorrhoids, } \\
\text { stomach disorders, } \\
\text { high blood pressure, } \\
\text { laxative, male and } \\
\text { female fertility issues, } \\
\text { tonic, aphrodisiac, } \\
\text { snake anti- venom }\end{array}$ & $\begin{array}{l}\text { Leaves, } \\
\text { seeds, } \\
\text { stembark }\end{array}$ & $\begin{array}{l}\text { Infusion, juice } \\
\text { extract, } \\
\text { mastication }\end{array}$ & 0.19 & 84 & 0.4 & 0.21 & s.s, s.e, s.w & $\begin{array}{l}\text { Ajibesin } e t \text { al. } \\
2008 \& 2012\end{array}$ \\
\hline Theobroma cacao L. BDU 343 & Sterculiaceae & Koko $(Y)$. & Cocoa & $\begin{array}{l}\text { Malaria, gingivitis, } \\
\text { toothaches, stimulant }\end{array}$ & $\begin{array}{l}\text { Stembark, } \\
\text { roots }\end{array}$ & Decoction & 0.09 & 88 & 0.2 & 0.22 & s.w & $\begin{array}{l}\text { Ariwaodo et al. } \\
2012 \text {, lyama \& } \\
\text { Idu } 2015\end{array}$ \\
\hline $\begin{array}{l}\text { Thymus vulgaris L. } \\
\text { BDU } 290\end{array}$ & Lamiaceae & $\begin{array}{l}\text { Nch-anwu (I.), } \\
\text { Efinrinwewe (Y.), Akuko } \\
\text { (IK.) }\end{array}$ & Curry leaf & $\begin{array}{l}\text { Antibiotic, } \\
\text { carminative, } \\
\text { dysentery, } \\
\text { stomachache, } \\
\text { hemorrhoid }\end{array}$ & Leaves & Mastication & 0.07 & 85 & 0.3 & 0.42 & s.e,s.w & $\begin{array}{l}\text { Ajibesin et al. } \\
2008 \& 2012\end{array}$ \\
\hline $\begin{array}{l}\text { Tithonia diversifolia (Helmsl) A. } \\
\text { Gray } \\
\text { BDU } 368\end{array}$ & Asteraceae & Jogbo-agbale (Y.) & $\begin{array}{l}\text { Tree } \\
\text { marigold }\end{array}$ & Malaria & Leaves & Decoction & 0.06 & 50 & 0.05 & 0.08 & s.w & Iyama \& Idu 2015 \\
\hline $\begin{array}{l}\text { Treculia africana Decne. } \\
\text { BDU } 278\end{array}$ & Moraceae & Afon $(Y)$. & $\begin{array}{l}\text { African } \\
\text { Bread fruit }\end{array}$ & $\begin{array}{l}\text { Malaria, diabetes, } \\
\text { laxatives, } \\
\text { antiheminthics, cough }\end{array}$ & Stembark & Decoction & 0.15 & 93 & 0.3 & 0.16 & s.w & $\begin{array}{l}\text { Akwaji et al. } \\
\text { 2017, Iyama \& } \\
\text { Idu 2015, Lawal } \\
\text { et al. 2010 }\end{array}$ \\
\hline $\begin{array}{l}\text { Trema orientalis (L.) Blume } \\
\text { BDU } 271\end{array}$ & Ulmaceae & $\begin{array}{l}\text { Afefe }(\mathrm{Y} .) \text {, Elemukien } \\
(\mathrm{O} .)\end{array}$ & $\begin{array}{l}\text { Charcoal } \\
\text { tree, } \\
\text { gunpowder } \\
\text { tree }\end{array}$ & $\begin{array}{l}\text { Malaria, debility, skin } \\
\text { disease, cough, } \\
\text { dysentry }\end{array}$ & $\begin{array}{l}\text { Leaves, } \\
\text { stembark }\end{array}$ & $\begin{array}{l}\text { Decoction, } \\
\text { infusion, poultice }\end{array}$ & 0.06 & 50 & 0.3 & 0.41 & s.w & $\begin{array}{l}\text { Ajibesin et al. } \\
2008 \text { \& } 2012, \\
\text { lyama \& Idu } 2015\end{array}$ \\
\hline $\begin{array}{l}\text { Trichilia monadelpha (Thonn) J.J. } \\
\text { De Wilde BDU } 279\end{array}$ & Meliaceae & Akorere $(\mathrm{Y})$. & & Malaria & Stembark & Infusion & 0.07 & 50 & 0.05 & 0.08 & s.w & Iyama \& Idu 2015 \\
\hline Tridax procumbens L. BDU 347 & Asteraceae & $\begin{array}{l}\text { Sabaruma (Y.), Mbuli } \\
\text { (IK.) }\end{array}$ & 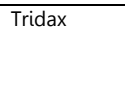 & $\begin{array}{l}\text { Malaria, skin diseases, } \\
\text { stomachache, back } \\
\text { ache }\end{array}$ & Leaves & $\begin{array}{l}\text { Decoction, } \\
\text { infusion }\end{array}$ & 0.11 & 82 & 0.2 & 0.18 & s.S, s.W & $\begin{array}{l}\text { Ariwaodo et al. } \\
\text { 2012, Iyama \& } \\
\text { Idu } 2015\end{array}$ \\
\hline $\begin{array}{l}\text { Triumfetta cordifolia A. Rich } \\
\text { BDU } 355\end{array}$ & Tiliaceae & Abiko (B.), Akee-eri (Y.) & & Malaria & Leaves & Decoction & 0.09 & 50 & 0.05 & 0.05 & s.s, s.W & Iyama \& Idu 2015 \\
\hline
\end{tabular}


Ethnobotany Research and Applications

\begin{tabular}{|c|c|c|c|c|c|c|c|c|c|c|c|c|}
\hline $\begin{array}{l}\text { Tussilago farfara L. } \\
\text { BDU232 }\end{array}$ & Asteraceae & Odoupo (EK.) & Coltsfoot & Miscarriage & Leaves & Decoction & 0.05 & 80 & 0.05 & 0.1 & s.s & $\begin{array}{l}\text { Ajibesin et al. } \\
2008 \& 2012\end{array}$ \\
\hline $\begin{array}{l}\text { Urena lobata L. } \\
\text { BDU } 290\end{array}$ & Malvaceae & $\begin{array}{l}\text { Odoazezo (I.), Akeriri } \\
\text { (Y.) }\end{array}$ & $\begin{array}{l}\text { Congo jute, } \\
\text { Caesar } \\
\text { weed }\end{array}$ & Malaria & Leaves & Decoction & 0.07 & 85 & 0.05 & 0.07 & s.e, s.w & lyama \& Idu 2015 \\
\hline $\begin{array}{l}\text { Uvaria chamae P. Beauv } \\
\text { BDU } 316\end{array}$ & Annonaceae & $\begin{array}{l}\text { Mmimi-ohia (I.), Mfee } \\
\text { (IK.) }\end{array}$ & $\begin{array}{l}\text { Cluster } \\
\text { pear }\end{array}$ & $\begin{array}{l}\text { Malaria, hemorrhoids, } \\
\text { boils, wound }\end{array}$ & $\begin{array}{l}\text { Leaves, } \\
\text { stembark, } \\
\text { roots }\end{array}$ & Decoction & 0.07 & 85 & 0.2 & 0.28 & s.e,s.s & lyama \& Idu 2015 \\
\hline $\begin{array}{l}\text { Vernonia amygdalina L. } \\
\text { BDU } 265\end{array}$ & Asteraceae & $\begin{array}{l}\text { Onugbu (I.), Origbo } \\
\text { (U.), Mbum(O-M.) }\end{array}$ & Bitter leaf & $\begin{array}{l}\text { Measles, smallpox, } \\
\text { chicken pox, diarrhea, } \\
\text { dysentery, } \\
\text { hemorrhoid, } \\
\text { inflammation, } \\
\text { diabetes, malaria, } \\
\text { constipation, } \\
\text { antifungal, } \\
\text { hypertension }\end{array}$ & $\begin{array}{l}\text { Leaves, leaf } \\
\text { sap, root } \\
\text { epidermis }\end{array}$ & $\begin{array}{l}\text { Hand crushing of } \\
\text { leaves to obtain } \\
\text { juice that is } \\
\text { either used as } \\
\text { decoction, } \\
\text { tincture, } \\
\text { maceration and } \\
\text { as poultice made } \\
\text { with crude chalk }\end{array}$ & 0.25 & 80 & 0.6 & 0.24 & s.e, s.s & $\begin{array}{l}\text { Alade \& Ajibesin } \\
2017 \text {, Lawal et al. } \\
2010\end{array}$ \\
\hline $\begin{array}{l}\text { Vitex doniana L. } \\
\text { BDU } 280\end{array}$ & Lamiaceae & Ufuru (O-M.) & Black plum & Fever, tooth decay & $\begin{array}{l}\text { Leaves, } \\
\text { stem }\end{array}$ & $\begin{array}{l}\text { Juice extract, } \\
\text { mastication }\end{array}$ & 0.13 & 73 & 0.1 & 0.07 & s.s & $\begin{array}{l}\text { Ajibesin et al. } \\
2008 \text { \& 2012, } \\
\text { Akwaji et al. } 2017\end{array}$ \\
\hline $\begin{array}{l}\text { Vitellaria paradoxa G.F. Gaertn- } \\
\text { BDU } 204\end{array}$ & Sapotaceae & $\begin{array}{l}\text { Okume (I.); Ori-oyo, } \\
\text { Emi-emi, emi (Y.) }\end{array}$ & Shea butter & Analgelsic & Fruit & Poultice & 0.09 & 66 & 0.05 & 0.06 & s.e, s.s, s.w & Lawal et al. 2010 \\
\hline $\begin{array}{l}\text { Ximenia americana L. } \\
\text { BDU } 280\end{array}$ & Olacaceae & $\begin{array}{l}\text { Out-ugba, Otua (I.), Igo } \\
\text { (Y.) }\end{array}$ & $\begin{array}{l}\text { Wild Lime, } \\
\text { Seaside } \\
\text { plum }\end{array}$ & Malaria & $\begin{array}{l}\text { Stembark, } \\
\text { roots }\end{array}$ & Decoction & 0.05 & 90 & 0.05 & 0.1 & s.e, s.w & $\begin{array}{l}\text { Ajibesin et al. } \\
2008 \text { \& } \\
2012 \text {,lyama \& Idu } \\
2015 \\
\end{array}$ \\
\hline $\begin{array}{l}\text { Xylopia aethiopica (Dunal) A. } \\
\text { Rich } \\
\text { BDU } 355\end{array}$ & Annonaceae & Enyi (K.), Eri-alamo (Y.) & $\begin{array}{l}\text { Ethiopian } \\
\text { pepper }\end{array}$ & $\begin{array}{l}\text { Malaria, womb } \\
\text { stabilization, Cough, } \\
\text { rheumatism, diarrhea, } \\
\text { dysentery, female } \\
\text { infertility, vaginal } \\
\text { infection }\end{array}$ & $\begin{array}{l}\text { Leaves, } \\
\text { fruit, seeds, } \\
\text { stembark }\end{array}$ & $\begin{array}{l}\text { Decoction, } \\
\text { tincture, } \\
\text { mastication, } \\
\text { poultice }\end{array}$ & 0.14 & 81 & 0.4 & 0.72 & s.s, s.w & $\begin{array}{l}\text { Akwaji et al. } \\
\text { 2017, Iyama \& } \\
\text { Idu 2015, } \\
\text { Soladoyeet al. } \\
2014\end{array}$ \\
\hline $\begin{array}{l}\text { Zanthoxylum leprieurï Guill. \& } \\
\text { Perr. } \\
\text { BDU253 }\end{array}$ & Rutaceae & Ata $(Y)$. & Fagara & Malaria, toothache & $\begin{array}{l}\text { Stembark, } \\
\text { roots }\end{array}$ & Tincture & 0.06 & 50 & 0.1 & 0.16 & s.w & Iyama \& Idu 2015 \\
\hline $\begin{array}{l}\text { Zanthozyllum zanthozy-loides } \\
\text { Lam. } \\
\text { BDU } 135\end{array}$ & Rutaceae & Ata (Y.) & Fagara & Asthma, antisickling & Stem, roots & $\begin{array}{l}\text { Decoction, } \\
\text { tincture, } \\
\text { mastication }\end{array}$ & 0.15 & 80 & 0.1 & 0.06 & s.w & Lawal et al. 2010 \\
\hline $\begin{array}{l}\text { Zingiber officinale Roscoe BDU } \\
244\end{array}$ & Zingiberaceae & Ajo, Ata-ile (Y.) & Ginger & $\begin{array}{l}\text { Malaria, female } \\
\text { infertility, cough, } \\
\text { catarrh }\end{array}$ & Rhizome & Decoction & 0.23 & 65 & 0.2 & 0.08 & s.w & $\begin{array}{l}\text { Alade \& Ajibesin } \\
2017 \text {, Iyama \& } \\
\text { Idu 2015, } \\
\text { Soladoyeet al. } \\
2014\end{array}$ \\
\hline
\end{tabular}




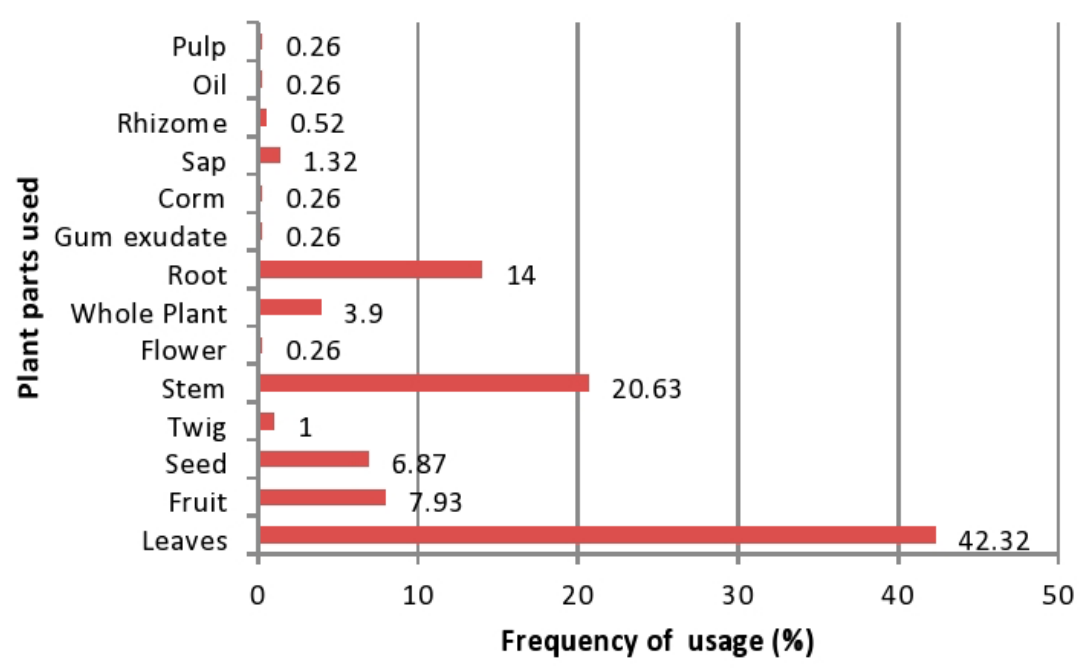

Figure 4. Frequency of plant parts usage

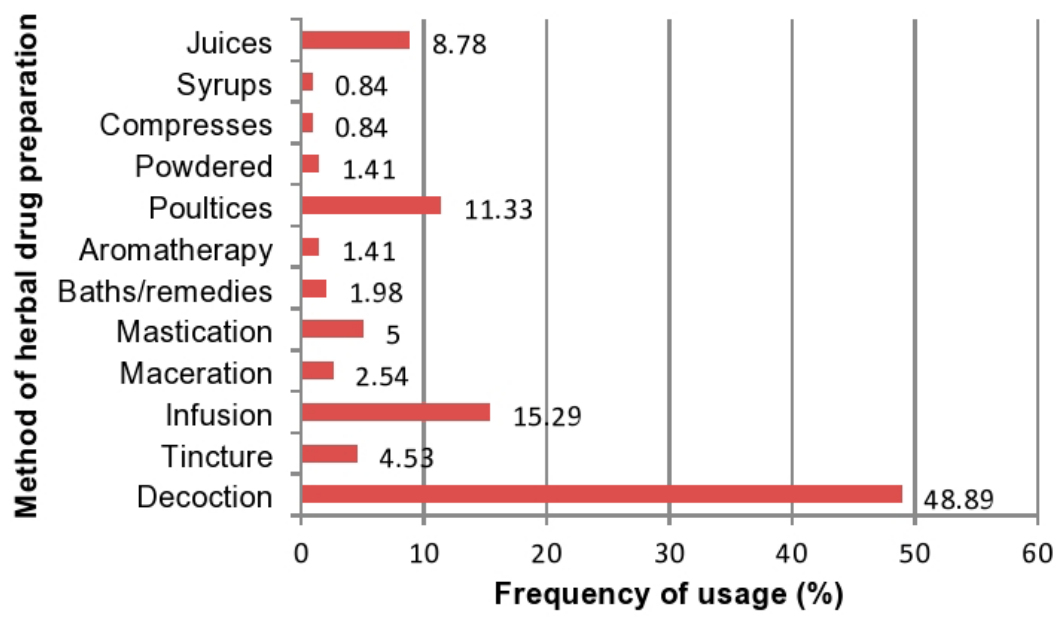

Figure 5. Method of preparation of herbal remedies.

\section{Frequency of plant therapeutic use}

Malaria showed a higher frequency (25.94\%) in plant therapeutic applications, followed by skin troubles (10.9\%), stomach ailments/ vermifuge (10.8\%), respiratory ailments (7.38\%), dental carries/ analgesic (4.86\%), infertility/ fibroid (4.5\%), antiviral (3.96\%), bone related issues and sexually transmitted disease each having a value of $3.6 \%$, hemorrhoids, eye troubles and heart disease each having a value of $2.7 \%$ respectively (Table 3 ).

\section{Relative frequency of citation (RFC)}

This is a statistical indicative of how users frequently cite a plant for different diseases. The RFC value for the species in southern Nigeria ranged between 0.02- 0.30 (Table 4). The highest RFC values for species was reported for $T$. occidentale (0.30), R. communis (0.27), A. precatorius and A. indica (0.26), C. limon, C. citratus, M. indica, M. oleifera, $O$. gratissimum and V. amygdalina (0.25 each ), A. melegueta, $A$. cordifolia, $C$. papaya, C. aurantifolia, D. tripetala, $P$. guajava and $Z$. officinale (0.23 each), A. montanus, A. boonei, A. djalonensis, E. guineense, E. senegalensis and $P$. osun (0.22 each). On the other hand, the highest RFC values for ethnomedicinal applications (Table 4) was reported for malaria (1.0), skin troubles, stomach troubles/ vermifuge (0.4 for each) and respiratory ailments (0.25). 
Table 4. Ethnomedicinal application of medicinal plants in Southern Nigeria

\begin{tabular}{|l|l|l|}
\hline Ethnomedicinal applications & $\begin{array}{l}\text { Frequency of citation } \\
\text { of ailments (FC) }\end{array}$ & $\begin{array}{l}\text { Relative frequency of citation } \\
\text { (RFC) RFC1/4 FC/N (N 1/4 200) }\end{array}$ \\
\hline Malaria & 200 & 1.0 \\
\hline Typhoid & 9 & 0.04 \\
\hline Respiratory diseases & 50 & 0.25 \\
\hline Heart diseases & 17 & 0.08 \\
\hline Bone issues & 23 & 0.12 \\
\hline Skin troubles & 77 & 0.4 \\
\hline Infertility/Fibroid & 30 & 0.15 \\
\hline Convulsions, fainting, stuttering, epilepsy & 12 & 0.06 \\
\hline Stomach troubles /vermifuge & 80 & 0.4 \\
\hline Diabetes & 16 & 0.08 \\
\hline Antiviral & 25 & 0.13 \\
\hline Antibacterial & 14 & 0.07 \\
\hline Antifungal & 12 & 0.06 \\
\hline Aphrodisiac & 5 & 0.03 \\
\hline Eye troubles & 17 & 0.09 \\
\hline Dental cares/ analgesic & 32 & 0.16 \\
\hline Sexually transmitted diseases & 23 & 0.12 \\
\hline Haemorrhoids & 17 & 0.09 \\
\hline Astringent & 3 & 0.02 \\
\hline Insanity/Insomnia & 6 & 0.03 \\
\hline Asperity, anemia, low immunity \& sickling & 20 & 0.1 \\
\hline Hair growth & 2 & 0.01 \\
\hline Dysmenorrheal, amenorrhea \& blennorrhagia & 12 & 0.06 \\
\hline Womb fixing \& poor lactation & 13 & 0.07 \\
\hline Anti-venoms & 8 & 0.04 \\
\hline Earache \& infection & 6 & 0.03 \\
\hline Vaginal/Urogenital infection & 6 & 0.03 \\
\hline Renal ailment/diuretic & 7 & 0.04 \\
\hline
\end{tabular}

\section{Informant consensus factor (ICF)}

Malaria and renal ailment (Table 5) had the highest value (0.28), followed by stomach troubles/vermifuge, womb fixing and poor lactation (0.25 each), earache/infection, insanity, insomnia and memory enhancer, vaginal and urogenital infection ( 0.20 each).

\section{Fidelity level (FL)}

Fidelity levels (FL) of the 236 species are presented in Table 3. The results are justified by the relative importance of the species to the indigenes in the study area and the therapeutic effectiveness of the plant species. High FL is an indicative of a particular disease in an area and the use of a species for its cure (Bibi et al. 2014). Species with high FL values are as follows: Elaeis guineense (99), Euphorbia hirta (97), Chrysophyllum cainto, Citrus limon, Cnestis ferruginea, Cymbopogon citratus, Funtumia africana, Gongronema latifolium, Terminalia superba (96 each), Dacryoides edulis, Dacus carota, Dialium guineense, Dracaena arborea, Eleusine indica, Jatropha Curcas, Mansonia altissima, Momordica charantia, Nauclea latifolia, Pergularia daemia, Scoparia dulcis (95 each), Alstonia boonei, Anthocleista djalonensis, Aspilla africana, Azadirachta indica, Cannabis sativa, Cocos nucifera, Erythrinia senegalensis, Morinda morindiodes, Pterocarpus osun, Sorghum bicolor, Stachytarpheta indica, Syndrella nodiflora, Telfairia occidentalis, Terminalia avicennioides, Ximenia americana (90 each) respectively.

\section{Relative popularity level (RPL)}

A total of 88 species were frequently cited by the respondents for treating various diseases but only 15 species had high relative popularity level (RPL) and these include: Abrus precatorius (0.8), Afromomum melegueta (0.7), Carica papaya, Newbouldia laevis and Vernonia amygdalina (0.6 each), Alchornea cordifolia, Ananas camosus, Baphia pubescence, Chromolaena odorata, Chrysophyllum cainito, Citrus aurantifolia, Citrus limon, Dalbergia lactea, Psidium guajava, Spondias mombin ( 0.5 each) respectively. Species most frequently cited by the respondents were accepted as popular while those not frequently cited were viewed as unpopular. 
Table 5. Informant consensus factor (ICF) values for 22 different diseases categories

\begin{tabular}{|c|c|c|c|c|c|}
\hline Category of diseases & $\begin{array}{l}\text { Number of } \\
\text { species }\end{array}$ & $\begin{array}{l}\text { Percentages } \\
\text { (\%) of species }\end{array}$ & $\begin{array}{l}\text { Number of } \\
\text { use citation }\end{array}$ & $\begin{array}{l}\text { Percentages } \\
\text { (\%) of use } \\
\text { citation }\end{array}$ & ICF value \\
\hline Malaria & 144 & 61 & 200 & 28.7 & 0.28 \\
\hline Typhoid & 8 & 3.38 & 9 & 1.3 & 0.11 \\
\hline Respiratory disorders & 41 & 17.37 & 50 & 7 & 0.18 \\
\hline Heart related ailment & 15 & 6.4 & 17 & 2.44 & 0.13 \\
\hline $\begin{array}{l}\text { Arthritis, rheumatism \& } \\
\text { bone cares }\end{array}$ & 20 & 8 & 23 & 3.29 & 0.13 \\
\hline Skin troubles & 61 & 26 & 77 & 11 & 0.22 \\
\hline Infertility & 25 & 11 & 30 & 4.3 & 0.17 \\
\hline $\begin{array}{l}\text { Convulsion, fainting, } \\
\text { epilepsy \& stuttering }\end{array}$ & 10 & 4 & 12 & 1.71 & 0.18 \\
\hline Stomach troubles & 60 & 25 & 80 & 11.49 & 0.25 \\
\hline $\begin{array}{l}\text { Asperity, anemia, low } \\
\text { immunity \& sickling }\end{array}$ & 17 & 7 & 20 & 2.86 & 0.15 \\
\hline $\begin{array}{l}\text { Dysmenorrheal, } \\
\text { amenorrhea \& } \\
\text { blennorrhea }\end{array}$ & 10 & 4 & 12 & 1.71 & 0.13 \\
\hline $\begin{array}{l}\text { Womb fixing \& poor } \\
\text { lactation }\end{array}$ & 10 & 4 & 13 & 1.86 & 0.25 \\
\hline Earache \& infection & 5 & 2.1 & 6 & 0.85 & 0.2 \\
\hline Diabetes & 14 & 6 & 16 & 2.29 & 0.13 \\
\hline Viral infection & 22 & 9 & 25 & 3.58 & 0.13 \\
\hline Eye trouble & 15 & 6.35 & 17 & 2.43 & 0.13 \\
\hline Tooth ache \& pain & 27 & 11 & 32 & 4.58 & 0.16 \\
\hline $\begin{array}{l}\text { Sexually transmitted } \\
\text { diseases }\end{array}$ & 20 & 8 & 23 & 3.95 & 0.13 \\
\hline Hemorrhoid & 15 & 6 & 17 & 2.44 & 0.13 \\
\hline $\begin{array}{l}\text { Insanity, insomnia, } \\
\text { memory enhancer }\end{array}$ & 5 & 2 & 6 & 0.86 & 0.2 \\
\hline $\begin{array}{l}\text { Vaginal \& Urogenital } \\
\text { infection }\end{array}$ & 5 & 2.1 & 6 & 0.86 & 0.2 \\
\hline Renal ailment & 6 & 2.5 & 7 & 1 & 0.28 \\
\hline
\end{tabular}

\section{Use value (UV)}

The relative importance of any species is revealed by its use value (Vendruscolo and Mentz 2006). A high use value was given by Carpolobia lutea (2.66), Heliotropium indicum (1.5), Citrus limon (1.41), Glyphaea brevis (1.3), Baphia pubescence (1.0), Xylopia aethiopica (0.72), Bixa orellana (0.66), Anthonotha macrophylla (0.58), Ceiba petandra, Combretum racemosum, Dalbergia lactea and Dioscoreae rotundata ( 0.5 each) respectively.

\section{Discussion}

Southern Nigeria has a high prevalence rate of malaria, typhoid, fevers, colds and chills, catarrh, flu, river blindness, respiratory disorders, eye problems and skin infections. In the past, programs established to eradicate numerous health problems in Nigeria have been inadequate. These have led to little improvement in the health status, especially in southern Nigeria. The study was undertaken to document information on the common plant resources employed in the ethnomedicinal practices of the indigenous people of the southern Nigeria, and to explore ways of sensitizing genuine conservation efforts.

Socio-demographic information and gender-based assessment on the use of medicinal plants by respondents

A high percentage was reported for persons above the age of 40 on the use of medicinal plant (82\%) when compared to those below that age (18\%). Also, herbal practitioners $<40(25 \%)$ and herbal practitioners $>40(75 \%)$, herbal vendors < $40(27 \%)$ and herbal vendors > 40 (73\%). This may be attributed to a lack of interest in 
ethnomedicine by the younger generation due to westernization and a poor communication between the older and younger generation. Similar trends in the area have been reported by lyama \& Idu 2015.

The statistical results show that both male and females make use of medicinal plants. However, women had a higher knowledge of medicinal uses of plants (52\%) in the study area than men (48\%). This agrees with earlier studies carried out in the area (lyamah \& Idu 2015).

\section{Regional biodiversity of southern Nigeria}

Biodiversity involves different spheres of biological variety including inter alia, species richness, taxonomic richness, genetic differences in each taxon, communities, ecosystems and landscapes inhabited by organisms and the indigenous knowledge of nature possessed by the indigenes living on the land (Kunwar et al. 2009, Ubom 2010). This review has attempted to assess the ethnomedicinal plant resources in southern Nigeria, highlighting the medicinal uses, mode of preparation and administration of herbal drugs. It also indicates the level of species richness as well as biodiversity in the study area. A total of 236 plant species belonging to 80 families were reported in this research review and these plants have shown certain adaptations to the region. Fabaceae family had the highest occurrence of plant species which numbered 30, Asteraceae 14 and Euphorbiaceae 13. South-Western Nigeria had the highest regional occurrence of plant species of about $47 \%$, south-south $31 \%$ and south-east $22 \%$. This suggests a relationship between the use of these medicinal plants, their distribution pattern and level of abundance in Southern Nigeria. Earlier research have reported legumes as having a high level of abundance and wide distribution across the ecological zones of Nigeria (Ayodele \& Yang 2012, lyama \& Idu 2015). Furthermore, ethnomedicinal uses of many species reported in this review have been reported by past research (Ariwaodo et al., 2012, Ajibesin et al., 2008, lyamah \& Idu, 2015). The high level of plant species in the South-west of Nigeria may stem from low rate of hydrocarbon mining and crude oil exploratory activities as in the south-south and, gullies and hills in the south-east. Also, it may be due to the high level of preservation of ancient sacred grooves, and forest landscapes or sanctuaries by the indigenes of south-western Nigeria for fetish or superstitious reasons.

\section{Therapeutic application of medicinal plants}

Medicinal plants play crucial role in the treatment of various ailments by the indigenous people in Southern Nigeria. Enormous health challenges and lack of adequate resources to access primary health care by the indigenes of southern Nigeria have made community elders with vast knowledge on the collection and administration of medicinal plants collaborate to provide treatment with the use of these plants. Basic factors that have promoted the use of these ethnomedicinal plants are affordability and availability, which are favored by the climate, soil type, swamps, water, and sunlight and so are easily cultivated within this region (Ajibesin et al. 2012).

Decoction (48.9\%) was the most common method of herbal drug preparation in the study area. Treasure et al. 2021 reported similar findings in the study area on the use of decoction prepared by boiling or steeping the plant material in water.

A higher frequency of use (25.94\%) was reported for malaria in plant therapeutic applications. Southern Nigeria is a coastal region noted for its tropical rainforest, high amount of rainfall and sunshine. A major challenge the area has to deal with is problems of poor drainage and waste disposal systems. The higher relative frequency of citation for malaria may arise from high level of infestation and numerous breeding grounds for mosquitoes in the area due to poor drainage and waste disposal systems. 
Table 6. Pharmacological applications of Ethnomedicinal Plants of southern Nigeria

\begin{tabular}{|c|c|c|c|c|c|}
\hline $\begin{array}{l}\text { Plants/ } \\
\text { Family }\end{array}$ & $\begin{array}{l}\text { Relative } \\
\text { frequency } \\
\text { of citation } \\
\text { (RFC) }\end{array}$ & $\begin{array}{l}\text { Ethnomedicinal applications/ } \\
\text { biological activities }\end{array}$ & Isolated Phytochemicals / Enzymes & $\begin{array}{l}\text { Stage of } \\
\text { clinical trial }\end{array}$ & References \\
\hline $\begin{array}{l}\text { Brachystegia eurycoma } \\
\text { (Fabaceae) }\end{array}$ & 0.17 & $\begin{array}{l}\text { Analgesic, anti-inflammatory, } \\
\text { antimicrobial, wound healing, } \\
\text { antioxidant, blood glucose } \\
\text { lowering, liver enzyme lipid } \\
\text { profile, gastrointestinal } \\
\text { modulating, growth inhibitory, } \\
\text { cytotoxic activities }\end{array}$ & - & Ivt & Atawodi 2017 \\
\hline $\begin{array}{l}\text { Tetracarpidium } \\
\text { conophorum } \\
\text { (Euphorbiaceae) }\end{array}$ & 0.19 & $\begin{array}{l}\text { Detoxification of venoms, anti- } \\
\text { diarrhea activity, male fertility } \\
\text { enhancing activities, antioxidant } \\
\text { activities, anti-chelating activity, } \\
\text { anti-ulcer and wound healing } \\
\text { activities, treats stomach } \\
\text { disorders, controls high blood } \\
\text { pressure }\end{array}$ & $\begin{array}{l}\text { Isolectinsfrom T. conophorum seed } \\
\text { extracts, polyphenolic compounds (3- } \\
\text { galactoside, lactoside, 3-pentoside, 3- } \\
\text { arabinoside, quercetin, p-coumaric-acid } \\
\text { and 3and 5-caffeoylquinic acids), } \\
\text { alkaloids, steroides and a moderate } \\
\text { concentration of tannins }\end{array}$ & Ivt & $\begin{array}{l}\text { Animashaun et al. 1994, Amaral et } \\
\text { al. 2004, Periera et al. 2007, } \\
\text { Olabinrin et al. 2010, Ezealisiji et al. } \\
2014 \text { a\&b, Ikpeme et al. 2014, } \\
\text { Nwachoko \& Jack 2015, Chikezie } \\
2017\end{array}$ \\
\hline $\begin{array}{l}\text { Azadirachta indica } \\
\text { (Meliaceae) }\end{array}$ & 0.26 & $\begin{array}{l}\text { Malaria fever, jaundice, syphilis, } \\
\text { anthelmintics, skin disease, } \\
\text { eczema, ringworm, emetic, } \\
\text { laxative, sore throat, antifungal, } \\
\text { immunostimulant, antibacterial, } \\
\text { antiviral, antimicrobial, measles }\end{array}$ & $\begin{array}{l}\text { Over } 135 \text { compounds have being } \\
\text { isolated and are mainly grouped into } \\
\text { two major classes: isoprenoids and its } \\
\text { derivatives- gedunin - possess anti- } \\
\text { malarial properties }\end{array}$ & Ivt, ivv & $\begin{array}{l}\text { Udeinya 1993, Dhara et al. 1999, } \\
\text { Adesegun \& Coker 2001, NNMDA } \\
2005 \text { \& 2008, Udeinya et al. 2006, } \\
\text { Odugbemi 2008, Alshawsh et al. } \\
2009\end{array}$ \\
\hline $\begin{array}{l}\text { Cymbopogon citratus } \\
\text { (Poaceae) }\end{array}$ & 0.25 & $\begin{array}{l}\text { Malaria, cough, sprains, } \\
\text { lumbago, stomach tonic, } \\
\text { stimulant, cold, chest pains, } \\
\text { rheumatic joints, diaphoretic, } \\
\text { diuretic, ringworm }\end{array}$ & $\begin{array}{l}\text { Terpenoids, aldehydes, Essential oils like } \\
\text { geranial }\end{array}$ & Ivt, ivv & $\begin{array}{l}\text { Bidla et al. 2004, Tchoumbougnang } \\
\text { et al. 2005, Odugbemi 2008), } \\
\text { NNMDA } 2008\end{array}$ \\
\hline $\begin{array}{l}\text { Mangifera indica } \\
\text { (Anacardiaceae) }\end{array}$ & 0.25 & $\begin{array}{l}\text { Malaria, yellow fever, anemia, } \\
\text { liver disease, diarrhea, diabetes, } \\
\text { skin lesion, high blood pressure, } \\
\text { hemorrhage, emmenagogue, }\end{array}$ & $\begin{array}{l}\text { Xanthone Glycosides - Mangiferin, } \\
\text { saponins, steroids and tannins }\end{array}$ & Ivt & $\begin{array}{l}\text { Awe 1998, NNMDA 2005\&2008, } \\
\text { Aiyeloja \& Bello 2006, Odugbemi } \\
2008\end{array}$ \\
\hline
\end{tabular}




\begin{tabular}{|c|c|c|c|c|c|}
\hline & & $\begin{array}{l}\text { insomnia, insanity, } \\
\text { anthelmintics, antimicrobials, } \\
\text { astringent, asthma, cough }\end{array}$ & & & \\
\hline $\begin{array}{l}\text { Carica papaya } \\
\text { (Caricaceae) }\end{array}$ & 0.23 & $\begin{array}{l}\text { Malaria, gonorrhea, syphilis, } \\
\text { amebic dysentery, roundworms, } \\
\text { abortifacients, emmenagogue, } \\
\text { diabetes, medicinal recipes, } \\
\text { hemostatic, hernia, infections of } \\
\text { urinogenital systems, } \\
\text { blennorrhagia, orchitis, papain } \\
\text { enzyme as meat tenderizer, } \\
\text { convulsion, mental disorder }\end{array}$ & Papain & Ivt & $\begin{array}{l}\text { Bhat \& Surolia 2001, Odugbemi, } \\
2008 \text {, Avwioro } 2010\end{array}$ \\
\hline $\begin{array}{l}\text { Psidium guajava } \\
\text { (Myrtaceae) }\end{array}$ & 0.23 & $\begin{array}{l}\text { Malaria fever, diarrhea, } \\
\text { stomachache, cough, laxative, } \\
\text { dysentery, irregular } \\
\text { menstruation sore throat, } \\
\text { laryngitis, skin ulcers, astringent, } \\
\text { antispasmodic, rheumatism, } \\
\text { epilepsy, cholera, convulsions, } \\
\text { mouth swelling }\end{array}$ & $\begin{array}{l}\text { Flavonoids, carbohydrates, saponins, } \\
\text { anthraquinones and terpenoids }\end{array}$ & Ivt & $\begin{array}{l}\text { Nundkumar \& Ojewole 2002, } \\
\text { NNMDA } 2005 \text { \& 2008, Obute } 2006\end{array}$ \\
\hline $\begin{array}{l}\text { Citrus aurantifolia } \\
\text { (Rutaceae) }\end{array}$ & 0.23 & $\begin{array}{l}\text { Fever, jaundice, stomache ache, } \\
\text { antimicrobials, abdominal ulcer, } \\
\text { gonorrhea, carminative, } \\
\text { hypertensive, flavoring agents, } \\
\text { measles, cough, toothache, } \\
\text { anthelmintics, scurvy, } \\
\text { insecticides }\end{array}$ & $\begin{array}{l}\text { Alkaloids, saponins, flavonoids and } \\
\text { glycosides }\end{array}$ & Ivt & $\begin{array}{l}\text { Obute 2006, Odugbemi et al. } 2007 \\
\text { \& 2008, NNMDA (2013), Bapna et al. } \\
2014 .\end{array}$ \\
\hline $\begin{array}{l}\text { Enantia chlorantha } \\
\text { (Annon-aceae) }\end{array}$ & 0.14 & $\begin{array}{l}\text { Malaria, typhoid fever, } \\
\text { antimicrobials, jaundice, } \\
\text { rickettsia, infective hepatitis, } \\
\text { hemostatic, uterus stimulant, } \\
\text { ulcer }\end{array}$ & Alkaloids, Phenolics & IVV & $\begin{array}{l}\text { NNMDA 2008, Odugbemi 2008, } \\
\text { Ayoade \& Musbau } 2010 .\end{array}$ \\
\hline $\begin{array}{l}\text { Vernonia amygdalina } \\
\text { (Asteraceae) }\end{array}$ & 0.25 & $\begin{array}{l}\text { Malaria, itching, ring worms, } \\
\text { weak erection, tonic, astringent, } \\
\text { diarrhea, antimicrobials, nervous } \\
\text { diseases, gingivitis, toothache, }\end{array}$ & $\begin{array}{l}\text { Bitter sesquiterpenes lactones } \\
\text { compounds, (i.e. vernolide, vernodalin, } \\
\text { hydroxyvernolide and the steroid }\end{array}$ & Ivt & $\begin{array}{l}\text { Tona et al. 2004, NNMDA 2005, } \\
\text { Odugbemi 2008, Omoregie et al. } \\
2011\end{array}$ \\
\hline
\end{tabular}




\begin{tabular}{|c|c|c|c|c|c|}
\hline & & $\begin{array}{l}\text { stomachache, impotency, } \\
\text { laxative, acute pains, piles, } \\
\text { rashes, hemostatic, diabetes, } \\
\text { pneumonia, enema }\end{array}$ & $\begin{array}{l}\text { related constituents), vernonioside B1 } \\
\text { and vernonoid B1 }\end{array}$ & & \\
\hline $\begin{array}{l}\text { Morinda lucida } \\
\text { (Rubiaceae) }\end{array}$ & 0.11 & $\begin{array}{l}\text { Malaria, typhoid fever, yellow } \\
\text { fever, cerebral congestion, } \\
\text { dysentery, dressing of wound, } \\
\text { diabetes, heart disease, } \\
\text { stomachache, purgative, emetic, } \\
\text { diuretic, jaundice, flatulence, } \\
\text { anti-cancer, low sperm count, } \\
\text { analgesic, laxative, trypanocidal } \\
\text { activity, ulcers, leprosy } \\
\text { gonorrhea }\end{array}$ & Damnacanthal & Ivt, ivv & $\begin{array}{l}\text { Awe \& Makinde 1998, NNMDA } 2005 \\
\text { \& 2008, Odugbemi et al. } 2007 .\end{array}$ \\
\hline $\begin{array}{l}\text { Ocimum gratissimum } \\
\text { (Lamiaceae) }\end{array}$ & 0.25 & $\begin{array}{l}\text { Fever, cough, convulsion, cold, } \\
\text { catarrh, bronchitis, colic, chest } \\
\text { pain, diarrhea, miscarriage, } \\
\text { nasal bleeding, insect repellant, } \\
\text { antimicrobials, anthelmintics, } \\
\text { hypertension, diabetes, piles, } \\
\text { antibacterial }\end{array}$ & Essential oils & Ivt & $\begin{array}{l}\text { Ngemenya et al. 2004, Olorunniyi \& } \\
\text { Morenikeji } 2013\end{array}$ \\
\hline $\begin{array}{l}\text { Chromolaena odorata } \\
\text { (Asteraceae) }\end{array}$ & 0.17 & $\begin{array}{l}\text { Malaria fever, typhoid fever, } \\
\text { diabetes, diuretic, rheumatic } \\
\text { pains, tumor, anti-inflammation, } \\
\text { stomach pain, antimicrobial, } \\
\text { dysentery, headache, toothache, } \\
\text { hemostatic, skin diseases }\end{array}$ & Quercetin-4'-methyl ether & IVV & $\begin{array}{l}\text { Odugbemi } 2007 \text { \& 2008, Ukpai \& } \\
\text { Amaechi 2012, Olorunniyi \& } \\
\text { Morenikeji 2013, Ezenyi et al. } 2014\end{array}$ \\
\hline $\begin{array}{l}\text { Anacardium occidentale } \\
\text { (Anacardiaceae) }\end{array}$ & 0.19 & $\begin{array}{l}\text { Malaria, typhoid fever, white } \\
\text { coating of the tongue, } \\
\text { toothache, sore gums, } \\
\text { dysentery, purgative, } \\
\text { elephantiasis, leprosy, } \\
\text { ringworms, scurvy, diabetes, } \\
\text { warts, anthelmintics, caries }\end{array}$ & Tannins & Ivt & $\begin{array}{l}\text { Odugbemi } 2007 \text { \& 2008, Razalia et } \\
\text { al. } 2008 \text {, Olorunniyi \& Morenikeji } \\
2013\end{array}$ \\
\hline $\begin{array}{l}\text { Ananas comosus } \\
\text { (Bromeliaceae) }\end{array}$ & 0.15 & $\begin{array}{l}\text { Malaria, Typhoid fever, cough, } \\
\text { anthelmintics, digestive }\end{array}$ & Bromelian & $1 \mathrm{vt}$, ivv & $\begin{array}{l}\text { Rajendra et al. 2012, Olorunniyi \& } \\
\text { Morenikeji } 2013\end{array}$ \\
\hline
\end{tabular}




\begin{tabular}{|c|c|c|c|c|c|}
\hline & & $\begin{array}{l}\text { problems, fibrinolytic action, } \\
\text { inhibiting platelet aggregation, } \\
\text { interfrring with the growth of } \\
\text { malignant cells, removing skin } \\
\text { (debridement), anti- } \\
\text { inflammatory, enhancing drug } \\
\text { absorption, purgative, } \\
\text { emmenagogue, vermifuge, } \\
\text { enzyme bromelaine for meat } \\
\text { tenderizer, treats angina } \\
\text { pectoris, bronchitis, sinusitis, } \\
\text { surgical trauma, osteoarthritis, } \\
\text { cardiovascular disease }\end{array}$ & & & \\
\hline $\begin{array}{l}\text { Persea americana } \\
\text { (Lauraceae) }\end{array}$ & 0.17 & $\begin{array}{l}\text { Malaria, hypertension, analgesic, } \\
\text { anti-inflammatory, anti- } \\
\text { convulsant, hypoglycaemic, } \\
\text { vasorelaxant, diuretic, parasitic } \\
\text { skin diseases, peptic ulcer, } \\
\text { aphrodisiac, insomnia, gastro- } \\
\text { intestinal disorders. }\end{array}$ & $\begin{array}{l}\text { 1,2,4-dihydroxy derivatives aliphatic } \\
\text { alcohols, called avocadenols }\end{array}$ & Ivt & Dike et al. 2012, Falodun et al. 2014 \\
\hline $\begin{array}{l}\text { Nauclea latifolia } \\
\text { (Rubiaceae) }\end{array}$ & 0.21 & $\begin{array}{l}\text { Febrile conditions, cough, } \\
\text { antifungal, thrush, jaundice, } \\
\text { piles, emetic, menstrual } \\
\text { disorders, stomach disorders, } \\
\text { measles, sore. }\end{array}$ & $\begin{array}{l}\text { Flavonoids, saponin, terpenoids and } \\
\text { tannin, Alkaloids. }\end{array}$ & Ivt & $\begin{array}{l}\text { Benoit-Vicala et al. 1998, Traore et } \\
\text { al. 2000, Odugbemi } 2008\end{array}$ \\
\hline $\begin{array}{l}\text { Alstonia boonei } \\
\text { (Apocynaceae) }\end{array}$ & 0.22 & $\begin{array}{l}\text { Malaria fever, anti-inflammatory, } \\
\text { stomach pain, tonic, } \\
\text { anthelmintics, yellow fever, } \\
\text { filaria worms, breast } \\
\text { development, antidote. }\end{array}$ & Alkaloid-alstonine, Terpenoids & $\begin{array}{l}\text { Ivt, subsequently } \\
\text { compounded } \\
\text { into tablets after } \\
\text { testing }\end{array}$ & $\begin{array}{l}\text { Tantchou et al. 1986, Okpekon et al. } \\
\text { 2004, NNMDA } 2005 \text { \& 2008, Obute } \\
\text { 2006, Odugbemi 2008, } \\
\text { Majekodunmi et al. } 2008\end{array}$ \\
\hline
\end{tabular}


The relative frequency of citation value for the species in southern Nigeria ranged between 0.02- 0.30. However, the highest RFC values for species were reported for T. occidentale (0.30). In ethnomedicinal applications, the highest relative frequency of citation value was reported for malaria (1.0). Relative frequency of citation in ethnomedicinal studies is used to select plant species having high medicinal values for intensive research and drug discovery (Malik et al. 2019).

Among the 64 most cited species in this study, 18 species have been investigated for their medicinal potencies based on reports obtained from previous studies on in vitro and in vivo activities of these plant species (Table 4). Earlier documentation have shown Azadirachta indica contains phytochemicals such as alkaloids, flavonoids, terpenoids, saponins, tannins, phenols and cardiac glycosides (Ayeni and Yahaya, 2010), Chromolaena odorata contains Quercetin-4'-methyl ether (Odugbemi, 2007 \& 2008), Cymbopogon citratus contains alkaloids, saponins, tannins, anthraquinones, steroids, phenols and flavonoids (Asaolu et al. 2009), and Tetracarpidium conophorum contains Isolectins isolated from seed extracts, polyphenolic compounds such as 3-galactoside, lactoside, 3pentoside, 3-arabinoside, quercetin, p-coumaric-acid and 3- and 5-caffeoylquinic acids, alkaloids, steroids and a moderate concentration of tannins (Amaral et al. 2004). In same vein, Vernonia amygdalina have been reported to contain Bitter sesquiterpenes lactones compounds such as vernolide, vernodalin, hydro-xyvernolide and the steroid related constituents, vernonioside B1 and vernonoid B1. Dike et al. 2012 stated various protective and therapeutic effects associated with these phytochemicals.

Plants therapeutic uses in southern Nigeria were mostly in the treatment of malaria, skin troubles, stomach ailments and vermifuge, respiratory ailment, dental cares and as analgesic. Plant species reported in the review are either administered singly or in combination with other plants of similar medicinal value in treatment of ailments to provide synergy for total elimination of disease-causing pathogens and aid quick recovery.

Decoction, infusion, poultices, and juices were the most used route in herbal drug preparation. However, plants had high incidence of oral consumption as decoction. The decoctions are usually boiled and drunk, this may indicate that the active ingredients found in most of the plants are not volatile.

Different parts of the medicinal plants are usually employed in preparation of herbal remedies. The parts of medicinal plants mostly used in herbal drug preparation were leaves, followed by stembark and root. Several studies have reported similar observation (Asase et al. 2010, Nguta et al. 2010, Ighere et al. 2011, Olorunnisola et al. 2013, Traore et al. 2013, lyama \& Idu 2015). A higher preference towards leaves may be resultant of common knowledge of leaves as the main photosynthetic organs in plants. Also, leaves act as storehouse for end products of photosynthesis or exudates which may contain more bioactive secondary metabolites for protection against predators such as Herbivores. Some of these compounds may be of medicinal value to the human body (Bhattarai et al. 2006). It has been established that the use of leaves poses less threat to the continued existence of plant species when compared to the use of underground parts like roots, stem, bark, or the use of entire plants (Zheng \& Xing, 2009, lyama \& Idu 2015).

The major issues that pose certain limitations on the use of plant as drugs are lack of information on the social, biochemical, and economic benefits that could be derived from the industrial utilization of medicinal plants, poor incentives for standardization of product, little information on the market potential and trading possibilities of these medicinal plants (Oladeji 2016).

\section{Previous studies or documentation}

Several studies have been made on the ethnomedicinal plants in selected areas (Ighere et al. 2011, Nwazuoma \& Dappa 2013, Odugbemi et al. 2017, Anowi \& Christian 2019, Chijindu et al. 2020, Chukwuma et al. 2020) or for specific uses (lyamah \& Idu 2015, Babawale et al. 2018, Ayeni \& Aliyu 2018, Chinedu \& Uyanwa 2019, Afolayan et al. 2020) across southern Nigeria in the past but not for the whole of southern Nigeria. Ethnomedicinal applications, biological activities, isolated phytochemicals, and active compounds as well as the status of scientific validation of the 18 listed plants, are shown in Table 4. These plants have been previously reported to contain various phytochemicals. Phytochemical extractions like Isolectins from Tetracarpidium conophorum seed extracts, polyphenolic compounds like 3-galactoside, lactoside, 3-pentoside, 3-arabinoside, quercetin, p-coumaric-acid and 3- and 5-caffeoylquinic acids (Tetracarpidium conophorum), tannins (Anacardium occidentale, Azadirachta indica, Mangifera indica, Nauclea latifolia, and Tetracarpidium conophorum), alkaloids (Alstonia boonei, Azadirachta indica, Nauclea latifolia, Citrus aurantifolia, Enantia chlorantha and Tetracarpidium conophorum), saponins (Citrus aurantifolia, Psidium guajava, Nauclea latifolia and Mangifera indica), glycosides (Citrus aurantifolia and Mangifera 
indica), terpenoids (Alstonia boonei, Cymbopogon citratus, Psidium guajava, Vernonia amygdalina and Nauclea latifolia), flavonoids (Citrus aurantifolia, Psidium guajava and Nauclea latifolia), polyphenolic compounds (Tetracarpidium conophorum), steroids (Tetracarpidium conophorum), essential oils (Cymbopogon citratus and Ocimum gratissimum).

\section{Conclusion}

Bioactivity and toxicity by in vitro and in vivo standard tests should be made on herbal drug extracts of the presented species for scientific validation of their efficacy, as well as isolation and possible identification of potentially active compounds. This holds a ray of hope for compounding of phtyo-drugs in an era of growing resistance of pathogenic organisms to chemically synthesized drugs. Increased anthropogenic activities in the coming years could emerge as a potential threat to conservation of biodiversity of plant species in southern Nigeria. Hence, a call is made for conservation of these species for their perpetuation.

\section{Declarations}

List of abbreviations: HIS: Health Information System, NHIS: National Health Insurance Scheme, S.E: South-East, S.S: South South, S.W: South West ,E: Edo, Y: Yoruba, I: Igbo, B: Bini, U: Urhobo, E: Efik, IK: Ikwerre, IB: Ibibio, EK: Ekpere, K: Kalabari, O: Ogoni, O-M: Oboso-Mbube, IJ: ljaw, BDU: Bioresources Development Centre Ubulu-Uku Herbarium, FC: citation frequency, N: total number of respondents, ICF: Informant consensus factor, Nur: Number of use citations for a disease category, Nt: Number of species used by informants in a given use category, FL: Fidelity level, RPL: Relative popularity value, UV: Use value.

Ethics approval and consent to participate: Informed prior consent was obtained from all respondents before commencement of the interview. Data were collected with respect to confidentiality, anonymity and consent.

Consent for publication: Not applicable

Availability of data and materials: The data was not deposited in public repositories.

Competing interests: The authors declare no conflict of interest.

Funding: This research did not receive any grant in any manner from funding agencies in the public, commercial, or not-for-profit sectors.

Authors' contributions: Enebeli-Ekwutoziam Katherine Chinwe, Aruah Blessing Chinenye, Ogbonna Blessing Onyemaechi, Eze Uju Judith, Egedeye-Fubura Fubaraye Sokari, Nwankwo Chigozie Fredrick, Oliseyenum Nelly Ifeoma, Udoha Ngozi Winfred, AfuyeTubosun Nathaniel, Asogwa Grace Nkechi: Study design, ethnomedicinal surveys conduction, active participation in structuring of the methodology, manuscript writing, data analysis and interpretation. Enebeli-Ekwutoziam Katherine Chinwe, Aruah Blessing Chinenye, Egedeye-Fubura Fubaraye Sokari, Study conception and supervision, methodology description and botanical identification. Chinweokwu Kris Ada, Anenih Farida Onose, lyamu James Ekhorutormwen, Oboti Feyinwa Deborah: Contribution to the study design and authorizations of different community heads to conduct the surveys. Nwaizu Nkechi, Ajabor James Ifeanyi, Ozadibe Ogechukwu Yvonne, Otunla Racheal Abosede, Okoronkwo Bertha Francis, Orji Miriam: Work supervising, contribution to methodology, manuscript improving and review-editing. All authors read, reviewed, and approved the manuscript.

\section{Acknowledements}

The authors wish to acknowledge the support provided by the management of Bioresources Development Centre Ubulu-Uku -National Biotechnology Development Agency. The authors extend gratitude to Mrs. EnebeliEkwutoziam, K.C. for editing the manuscripts and carrying out statistical analysis of research data, and Ms. Aruah, C.B. for proofreading the manuscripts.

\section{Literature cited}

Abd El-Ghani, MM, 2016. Traditional medicinal plants of Nigeria: an overview. Agriculture and Biology Journal of North America 7(5):220-247. doi:10.5251/abjna.2016.7.5.220.247.

Abebe, D, Ayehu, A. 1993. Medicinal Plants and Enigmatic Health Practices of Northern Ethiopia. B.S.P.E., Addis Ababa, Ethiopia.

Abere, TA, Okoye, J, Agroreyo, FO. 2015. Antisickling and toxicological evaluation of the leaves of Scoparia dulcis Linn. (Scrophllariaceae). BMC Complementary and Alternative Medicine Journal 15: 414. doi:10.1186/s12906-0150928-5. 
Adesegun SA, Coker HAB. 2001. Plants used in tradition medicine against malaria. Nigerian Journal of Pharmacology 32:50-62.

Adeyemo DO. 2005. Local Government and Health care delivery in Nigeria: A case study. Journal of Human Ecology 18:149-60. doi: 10.1080/09709274.2005.11905822.

Afolayan ID, Sulaiman KA, Okunade WT. 2020. Ethnobotanical survey of plants used in cancer therapy in Iwo and Ibadan, South-Western of Nigeria. Journal of Pharmacy and Pharmacognosy Research 8(5):346367.http://jppres.com/jppres (Accessed 22/07/2021).

Aiyeloja AA, Bello OA. 2006. Ethnobotanical potentials of common herbs in Nigeria: a case study of Enugu state. Journal of Educational Research and Reviews 1:16-22. http://academicjournals.org/ERR2 (Accessed 22/07/2021).

Aja PM, Ugwu OPC, Keke K, Ibere JB, Ekpono EU. 2017. Phytochemical Analysis of Senna occidentalis leaves. IDSOR Journal of Applied Sciences 2(1):75-91. www.idosr.org (Accessed 1/08/2021).

Ajibesin KK, Danladi NB, Uwemedimo FU. 2012. Ethnomedicinal survey of plants used by the indigenes of Rivers State of Nigeria. Pharmaceutical Biology, 50 (9), 1123-1143. doi: 10.3109/13880209.2012.661740.

Ajibesin KK, Ekpo BA, Bala DN, Essien EE, Adesanya SA. 2008. Ethnobotanical survey of Akwa Ibom State of Nigeria. Journal of Ethnopharmacology 115:387-408. doi: 10.1016/j.jep.2007.10.021.

Ajuru MG. 2018. Ethnobotanical inventory of Oguru-ama Town, Degema Local Government Area, Rivers State, Nigeria. Journal of Advances in Biology and Biotechnology 19(2):1-13. doi: 10.9734/JABB/2018/13576.

Akinyemi O, Oyewole SO, Jimoh KA. 2018. Medicinal plants and sustainable human health: a review. Horticulture International Journal 2(4):194-195. doi: 10.15406/hij.2018.02.00051.

Akwaji PI, Eyamand EO, Bassey RA. 2017. Ethnobotanical Survey of Commonly Used Medicinal Plants in Northern Cross River State, Nigeria. World Scientific News 70(2):140-157. www.worldscientificnews.com (Accessed 1/8/2021).

Alade G, Oladele A, Okpako E, Ajibesin K, Olanrewaju A. 2018. A Survey of plants used for family planning in Bayelsa State, southern Nigeria. Journal of Intercultural Ethnopharmacology 7(1):25-44. doi:10.5455/jice.20171202114930.

Alade GO, Ajibesin, KK. 2017. Herbal medicine cleric's knowledge in sub-urban centre in Niger-Delta Nigeria: a pilot study. Journal of Pharmacy and Pharmacognosy Resesearch 5 (4):200-216. http://jppres.com/jppres (Accessed $1 / 8 / 2021)$.

Alshawsh MA, Mothana RA, Al-shamahy HA, Salah F, Lindequist AU. 2009. Assessment of anti-malaria activity against Plasmodium falciparium and phytochemical screening of some Yemeni medicinal plants. Evidence Based. BMC Complementary and Alternative Medicine 6:453-456. doi: 10.1093/ecam/nem148.

Amaral JS, Seabra RM, Andrade PB, Valentao P, Pereira JA, Ferreres F. 2004, 'Phenolic profile in the quality control of walnut (Juglans regia L.) leaves. Food Chemistry 88:373-379. doi: 10.1016/j.foodchem.2004.01.055.

Alexiades MN, Sheldon JW. 1996. Selected guidelines for ethnobotanical research: A Field Manual. Boranx, NY: the New York Botanical Garden, U.S.A.

Ali-Shtayeh MS, Yaniv Z, Mahajna J. 2002. Ethnobotanical survey in the Palestinian area: a classification of the healing potentials of medicinal plants. Journal of Ethnopharmacology 71:221-232. doi: 10.1016/s03788741(00)00316-0.

Animashaun T, Togun RA, Hughes RC. 1994. Characterization of isolectins in Tetracarpidium conophorum seeds (Nigerian Walnut), Glycoconj Journal 11(4):299-303. doi: 10.1007/BF00731202.

Anowi CF, Christian UI. 2019. Ethnobotanical surveys of medicinal plants used by the natives of Umuahia, Abia State, Nigeria for the management of diabetes. IOSR Journal of Pharmacy and Biological Sciences 14:05-37. http://www.iosrjournals.org/iosr-jpbs/papers/Vol14-issue5/Series-1/B1405010537.pdf (Accessed 30/6/2021).

Ariwaodo JO, Chukwuma EC, Adeniji KA, 2012. Some medicinal plant species of Asamagbe stream bank vegetation, Forestry Research Institute of Nigeria, Ibadan. Ethnopharmacology Research and Applications10:541-549. https://www.researchgate.net/publication/281443566_13_JO_Ariwaodo_EC_Chukwuma_KA_Adeniji_2012_Some_M edicinal_Plant_Species_of_Asamagbe_Stream_Bank_Vegetation_Forestry_Research_Institute_of_Nigeria_lbadan_Eth nobotany_Research_Applications_10_541-549 (Accessed 1/8/2021). 
Asase A, Akwetey GA, Achel DC. 2010. Ethnopharmacological use of herbal remedies for the treatment of malaria in the Dangme West District of Ghana. Journal of Ethnopharmacology129:367-376. doi: 10.1016/J.JEP.2010.04.001.

Asaolu, MF, Oyeyemi, OA, Olanlokun, JO. 2009. Chemical compositions, phytochemical constituents, and in vitro biological activity of various extracts of Cymbopogon citratus. Parkistan Journal of Nutrition 8(12):1920-1922. doi: 10.3923/pin.2009.1920.1922.

Atawodi SE, Illiemene UDE. 2017. Evaluation of Brachystergia eurycoma Harmsseed dietary inclusion in the prevention of colon carcinogenesis. Annals of oncology 259. doi: 10.1093/annonc/mdx261.257.

Avwioro G. 2010. Effectiveness of some medicinal plant decoction in the treatment of malaria in Nigeria. Annals of Biology Research 1 (2):230-237. www.scholarsresearchlibrary.com (Accessed 1/8/2021).

Awe SO. 1998. Anti-plasmodial and antipyretic screening of Mangifera indica extract. Phytotherapy Research 12:437. doi: 10.1002/(SICI)1099-1573(199809)12:6<437::AID-PTR313>3.0.CO,2-C.

Awe SO, Makinde JM. 1998. Effect of petroleum ether fractions of Morinda lucida on, Plasmodium berghei in mice. Pharmaceutical Biology 36:301-304. doi: 10.1076/phbi.36.4.301.4581.

Ayeni EA, Aliyu N. 2018. Ethnobotanical survey and documentation of healing river sources among the Yoruba people (ljesha Land) Nigeria. Journal of Complementary Medicine Research 8:59-70. doi: 10.5455/jcmr.20180504090258.

Ayeni KE, Yahaya SA. 2010. Phytochemical screening of three medicinal plants: neem leaf (Azadirachta indica), hibiscus leaf (Hibiscus rosa-sinensis) and Speargrass leaf (Imperata cylindrical). Continental Journal of Pharmaceutical Science 4:47-50. https://wiloludjournal.fandom.com/wiki/Continental_J_Pharmaceutical_ Sciences_Volume_4_(2010) (Accessed1/8/2021).

Ayoade AA, Musbau AA. 2010. Antimalaria bioactivity of Enantia chlorantha stem bark. Medicinal Plants: Phytochemistry, Pharmacology and Therapeutics 1 (2010), 441-447. https://basicmedicalkey.com/bioactivity-ofenantia-chlorantha-stem-bark/ (Accessed 1/8/2021)

Ayodele AE, Yang Y. 2012. Diversity and distribution of vascular plants in Nigeria. Qingdao Publishing House, Qingdao, China.

Babawale B, Taiye FR, Adetunji OS. 2016. Ethnobotanical survey of plants used as memory enhancer in 3 states of southwestern Nigeria. Journal of applied pharmaceutical science 6(09):209-214. doi: 10.7324/JAPS.2016.60931.

Balick MJ, Cox PA. 1996. Plants, culture, and people. WH Freeman \& Co., Scientific American New York, United States of America.

Bapna S, Ramaiya M, Chowdhary A. 2014. Brine shrimp toxicity and invitro antimalarial activity of Citrus aurantifolia (Christm.) Swingle against Plasmodium falciparum 3D7. IOSR Journal of Pharmaceutical and Biological Science 9 (5):24-27. doi: 10.9790/3008-09512427.

Benoit-Vicala F, Valentina A, Cournaca V, Pelissierb Y, Malliea M, Bastidea JM.1998. In vitro anti-plasmodial activity of stem and root extracts of Nauclea latifolia S.M. (Rubiaceae). Journal of Ethnopharmacology 61:173-178. doi: 10.1016/s0378-8741(98)00036-1.

Bhat GP, Surolia N. 2001.In vitro anti-malaria activity of extracts of three plants used in the traditional medicine of India. Am. J. Trop. Med. Hyg 65(4):304-8. doi: 10.4269/ajtmh.2001.65.304.

Bhattarai S, Chaudhary RP, Taylor RS. 2006. Ethnomedicinal plants used by the people of Manang district, central Nepal. Journal of Ethnobiology and Ethnomedicine 2:41 doi: 10.1186/1746-4269-2-41.

Bibi T, Ahmad M, Tareen RB, Tareen NM, Jabeen R, Rehman S, Sultana S, Zafar M, Yaseen G. 2014. Ethnobotany of medicinal plants in district Mastung of Balochistan province- Pakistan. Journal of Ethnopharmacology 157:79-89. doi: 10.1016/j.jep.2014.08.042.

Bidla G, Titanji VPK, Jako B, Ghazali GE, Bolad A, Berzins, K. 2004. Anti-plasmodial activity of seven plants used in African folk medicine. Indian Journal of Pharmacology 36, 245-246. http://www.bioline.org.br/request?tc08032 (Accessed 1/8/2021). 
Bryce J, Boschi-Pinto C, Shibuya K. 2005. Who estimates the cause of death in children? Lancet PubMed, 365:114752. doi: 10.1016/S0140-6736(05)71877-8.

Burkhill HM. 1995. The useful plants of west tropical Africa. $2^{\text {nd }}$ Edition, Volume 3, Royal Botanic Gardens, Kew, Richmond, UK.

Chijindu PCl, Okpoma, MO, Atubi O. 2020. Ethnobotanical survey of medicinal plants used in Erhuwaren community in Ughelli south local government area of Delta state. Unilag Journal of Medicine, Science and Technology (UJMST) (CEBCEM Special Edition) 8(1):176-206. http://journals.unilag.edu.ng/index.php/ujmst/citationstylelanguage/ get/turabian-fullnote-bibliography?submissionld=1025 (Accessed 1/8/2021).

Chikezie UN. 2017. Phytochemical and proximate composition of Tetracarpidium conophorum (African Walnut) seeds. International Journal of Research Studies in Biosciences 5(10):25-31. doi:10.24297/JBT.V7I1.6411.

Chinedu F, Uyanwa IC. 2019. Ethnobotanical survey of medicinal plants used by the natives of Umuahia, Abia State, Nigeria for the management of diabetes. IOSR Journal of Pharmacy and Biological Sciences 14(5):05-3. doi: 10.9790/3008-1405010537.

Chukwuma EC, Chukwuma DM, Adio AF. 2020. Flora diversity of ljero Local Government Area of Ekiti State, Southwestern Nigeria. Tropical Plant Research 7(1):5-64. doi: 10.22271/tpr.2020.v7.i1.009.

Clark TW, Daneshvar C, Pareek M, Perera N, Stehenson I. 2010. Enteric fever in a United Kingdom regional infectious diseases unit. A 10 years retrospective review. Journal of Infection 60(2):91-8. doi: 10.1016/j.jinf.2009.11.009.

Dhara R, Zhang K, Talwar GP, Gargb S, Kumara N. 1999. Inhibition of thegrowth and development of asexual and sexual stages of drug-sensitive and resistant strains of the human malaria parasite Plasmodium falciparum by Neem (Azadirachta indica) fractions. J. Ethnopharmacol. 61, 31-39. doi: 10.1016/s0378-8741(98)00012-9.

Dike IP, Obembe OO, Adebiyi FE. 2012. Ethnobotanical survey for potential anti-malarial plants in South-Western Nigeria. Journal of Ethnopharmacology 144:618-626. doi: 10.1016/j.jep.2012.10.002.

Duchelle AE. 2007. Observations on natural resource use and conservation by the shuar in Ecuador's cordillera Del condor. Ethnobotany Research and Application 5:5-23. doi: s10.17348/ERA.5.0.5-23.

Eke P. 1999. Intergroup Relation In: Introduction to Nigerian socio-cultural heritage. Edited by Anikpo, MOC, Atemie JD, Osia International publishing company, Nigeria.

Ezealisiji KM, ljeomah SC, Agbo MO. 2014a. Anti-ulcer activity of African walnut, Tetracarpidium conophorum nuts against gastric ulcers in Rats. Asian Pacific Journal of Tropical Disease 4(1):670-673. doi: 10.1016/S22221808(14)60772-6.

Ezealisiji KM, Omotosho AE, Udoh R, Agbo MO. 2014b. Wound healing activity of $n$-hexane and methanol extracts of Tetracarpidium conophorum (Mull. Arg.) Hutch (African Walnut) in Wistar rats. Malaysia Journal of Pharmaceutical Sciences 12(1):79-88. https://www.researchgate.net/publication/273313981_ wound_healing_activity_of_n-hexane_and_methanol_extracts_of_tetracarpidium_conophorum_mull_arg_hutch_ african_walnut_in_wistar_rats (Accessed 7/6/2021).

Ezenyi IC, Salawu OA, Kulkarni R, Emeje M. 2014. Antiplasmodial activity aided isolation and identification of quercetin-4'-methyl ether in Chromolaena odorata leaf fraction with high activity against chloroquine resistant Plasmodium falciparum. Parasitology Research 113(12):4415-4422. doi :10.1007/s00436-014-4119-y.

Ezenduka CC, Fallieros DR, Godman BB. 2017. Evaluating the treatment cost for uncomplicated malaria at a public healthcare facility in Nigeria and its implications. PharmacoEconomics 1:185-194. doi: 10.1007/s41669-017-00218.

Falodun A, Imieje V, Erharuyi O, Ahomafor J, Jacob MR, Khan SI, Hamann MT. 2014. Evaluation of three medicinal plants extracts against Plasmodium falciparium and selected microorganisms. African Journal of Traditional, Complementary and Alternative Medicine 11(4):142-146. doi: 10.4314/ajtcam.v11i4.22.

Gbille ZO, Adesina SK. 1986. Nigerian flora and its pharmaceutical potentials. Journal of Ethnopharmacology 19:116. doi: 10.1016/0378-8741(87)90135-8.

Giday M, Asfaw Z, Elmqvist T, Woldu Z. 2003. An ethnobotanical study of medicinal plants used by the Zay people in Ethiopia. Journal of Ethnopharmacology 85:43-52. doi: 10.1016/s0378-8741(02)00359-8. 
Gillespie S. 2018. What are intestinal worms? In: Healthline, Reviewed by Sampson S. https://www.healthline.com/health/intestinal-worms (Accessed 3/8/2021).

Gill LS. 1992. Ethnomedicinal uses of Plants in Nigeria.Uniben Press, Benin City, Nigeria.

Heinrich M, Ankli A, Frei B, Weimann C, Sticher O. 1998. Medicinal plants in Mexico: Healers' consensus and cultural importance. Social Science \& Medicine 47(11):1859-1871. doi: 10.1016/s0277-9536(98)00181-6.

Hostellmann K, Marston A. 2002. Twenty years of research into medicinal plants: results and perspectives. Phytochemistry Reviews 1:275-285. doi:10.1023/A:1026046026057.

Huntington HP.2000. Using traditional ecological knowledge in science: methods and applications. Ecological applications 10(5):1270-1274. doi: 10.2307/2641282.

Hutchinson J, Dalziel JM. 1954. Flora of West Tropical Africa. Vol 1 part 1, $2^{\text {nd }}$ Edition. The White Frairs Press, Limited, London, UK.

Hutchinson J, Dalziel JM. 1958. Flora of West Tropical Africa.Volume 1 part 2. The White Frairs Press, Limited, London, UK.

Hutchinson J, Dalziel JM. 1968. Flora of West Tropical Africa. Volume 3 part 1. The White Frairs Press, Limited, London, UK.

Idowu OA, Soniran OT, Ajana O, Aworinde DO. 2009. Ethnobotanical survey ofanti-malarial plants used in Ogun State, Southwest Nigeria. African Journal of Pharmacy and Pharmacology 4:055-060. doi: 10.5897/AJPP.9000138.

Idu M, Ndukwu BC, Osemwegie OO. 2007. Ethnofloristic studies of Ethiope Council Area of Delta State, Nigeria. Journal of Plant Science 2:1-13. doi: 10.3923/jps.2007.1.13.

Idu M, Onyibe HI, Timothy O, Erhabor JO. 2008. Ethnomedicinal flora of otuo people of Edo State, Nigeria. Asian Journal of Plant Science 7:8-12. doi: 10.3923/ajps.2008.8.12.

Ighere, DA, Ajiboye TO, Edagbo DE, Borokini TI, Alowonle AA, Micheal C,Giwa A, Adeyemo A. 2011. Ethnobotanical survey of local herbs used for the treatment of malaria fever among the Urhobo people in Delta State, Nigeria. International Journal of Current Research 3:336-339. http://www.journalcra.com (Accessed 1/8/2021).

Ikpeme EV, Ekaluo UB, Udensi O, Ekerette EE, Ekpo PB, Asuquo BO. 2014. Sperm quality and hormone profile of male albino rats fed with seeds of African walnut (Tetracarpidium conophorum, Mull.). Annual Research and Review in Biology 4(9):1379-1386. doi: 10.9734/ARRB/2014/5426.

Iwu MM. 1993. Handbook of African Medicinal Plants. CRC Press, Inc., Corporate Blvd., Florida, USA.

lyamah PC, Idu M. 2015.Ethnomedicinal survey of plants used in the treatment of malaria in Southern Nigeria. Journal of Ethnopharmacology 173:287-302. doi: 10.1016/j.jep.2015.07.008.

Jimoh A, Sofola O, Petu A, Okorosobo T. 2007. Quantifying the economic burdenof malaria in Nigeria using the willingness to pay approach. Cost Effect. ResourceAllocation 5:6-13. doi: 10.1186/1478-7547-5-6.

Kajang YG, Keswet LA. 2006. Health challenges in the present democratic era in Nigeria: the place of technology. International Journal of Technical Research and Applications 4 (1):124-129. http://hdl.handle.net/123456789/1443 (Accessed 7/6/2021).

Keay RWJ. 1989. Trees of Nigeria. Clarendon Press, Oxford, Great Britain.

Khan TI, Dular AK, Deepika MS. 2003. Biodiversity conservation in Thar Desert, with emphasis on endemic and medicinal plants. Environmentalist 23:137-144. doi: 10.1023/A:1024835721316.

Kunwar RM, Uprety Y, Burlakoti C, Chowdhary CL, Bussmann RW. 2009. Indigenous use and ethnopharmacology of Medicinal plants in far-West Nepal. Ethnobotany Research and Applications7:5-28. https://www.researchgate.net/publication/229062180_Indigenous_Use_and_Ethnopharmacology_of_Medicinal_Pla nts_in_Far-West_Nepal (Accessed 1/8/2021).

Lawal IO, Uzokwe NE, Igboanugo ABI, Adio AF, Awosan EA, Nwogwugwu JO, Faloye B, Olatunji BP, Adesoga AA. 2010. Ethnomedicinal information on collation and identification of some medicinal Plants in Research Institutes 
of South-West Nigeria. African Journal of Pharmacy and Pharmacology 4 (1):001-007. https://academicjournals.org/journal/AJPP/edition/January_2010 (Accessed 3/8/2021).

Majekodunmi SO, Adegoke OA, Odeku OA. 2008. Formulation of the extract of the stem bark of Alstonia booneias tablet dosage form. Tropical Journal of Pharmaceutical Research 7(2):987-994. doi: 10.4314/tjpr.v7i2.14683.

Menizibeya OW. 2011. The Nigerian health care system: Need for integrating adequate medical intelligence and surveillance systems. Journal of pharmacy and bioallied sciences 3(4): 470-478. doi: 10.4103/0975-7406.90100.

Murray P. 2013. Medical microbiology, 7th Ed. Elsevier Saunders, Philadelphia, U.S.A.

Mustapha AA, Fawibe OO, Ajiboye AA, Agoobla DA. 2014. Ethnobotanical survey of medicinal plants used in the treatment of diabetes in Irepodun Local Government of Osun State of Nigeria. Greener Journal of Biological Sciences 4(2):059-068. doi: 10.15580/GJBS.2014.2.010314006.

National Health insurance scheme decree No35 of 1999 Laws of the Federation of Nigeria. https://www.ilo.org (Accessed 1/8/2021).

Ndukwu BC, Ben-Nwadibia NB. 2005. Ethnomedicinal aspects of plants used as spices and condiments in the Niger Delta Area of Nigeria. Ethnobotanical Leaflets 19(10):1-32 https://opensiuc.lib.siu.edu/ebl/vol2005/iss1/10 (Accessed 1/8/2021).

Nguta JM, Mbaria JM, Gakuya DW, Gathumbic PK, Kiamad SG. 2010. Traditional antimalarial phytotherapy remedies used by the South Coast community, Kenya. Journal of Ethnopharmacology 131:256-267. doi: 10.1016/j.jep.2010.06.031.

Ngemenya MN, Tane P, Berzins K, Titanji VPK. 2004. Antiplasmodial activity of some medicinal plants used in Cameroon: preliminary toxicity studies of highly active plant extracts, in proceedings of the $11^{\text {th }}$ Annual Conference of The Cameroon Bioscience Society, December 2004.

Nigeria Health Information System Policy. FMOH, September, 2014. https://ehealth4everyone.com/ wpcontent/uploads/2015/09/Nig-Health-Info.pdf (Accessed 1/8/2021)

Nigeria National Health Conference Communique, Abuja Nigeria. https://www.ngnhc.org (Accessed 18/11/2020).

Nigeria Natural Medicine Development Agency (NNMDA). 2005. Medicinal Plants of Nigeria South-west Nigeria, vol. 1, NNMDA, Lagos, Nigeria.

Nigeria Natural Medicine Development Agency (NNMDA). 2008. Medicinal Plants of Nigeria Southeast Zone, vol. I, Lisinda Consulting, Lagos, Nigeria.

Nigeria Natural Medicine Development Agency (NNMDA). 2013. Medicinal Plants of Nigeria South-South Zone, vol. I, Koredex Associates, Lagos, Nigeria.

Nnadozie O. 2015. Estimating malaria burden in Nigeria: a geostatistical modeling approach. Geospatial Health 205(10):306. doi: 10.4081/gh.2015.306.

Nundkumar N, Ojewole JAO. 2002. Studies on antiplasmodial properties of some South African plants used as antimalaria remedies in Zulu folk medicine. Experimental and Clinical Pharmacology 24(7):397-401. doi: 10.1358/mf.2002.24.7.696540.

Nwachoko N, Jack IR. 2015, Phytochemical screening, and anti-diarrhea activities of Tetracarpidium conophorum induced in albino rats. Sky Journal of Biochemistry Research 4(4):21-24. http://www.skyjournals.org/SJBR (Accessed 2/8/2021).

Nwauzoma AB, Dappa MS. 2013. Ethnobotanical studies of Port Harcourt metropolis, Nigeria. International Scholarly Research Notices Botany1-11. doi:10.1155/2013/829424.

Obute G. 2005. Ethnomedicinal plant resources of south-eastern Nigeria. Ethnobotanical Leaflets 2005(1):5. https://openiuc.lib.siu.ed/ebl/vol/2005/iss1/5 (Accessed 30/6/2021)

Obute GC. 2007. Ethnomedicinal plant resources of South Eastern Nigeria. African Journal of Interdisciplinary Studies 3(1):90-94. https://opensiuc.lib.siu.edu/ebl/vol2005/iss1/5 (Accessed 9/12/2020). 
Obute GC, Osuji LC. 2002. Environmental awareness and dividends: a scientific discourse. African Journal of Interdisciplinary studies 3(1):90-94.

Odugbemi T. 2008. A Textbook of Medicinal Plants from Nigeria Lagos. University of Lagos Press, Nigeria.

Odugbemi TO, Oduunayo R, Akinsulire I, Albinu E, Fabeku PO. 2007. Medicinal plants useful for malaria therapy in okeigbo, Ondo State, southwest, Nigeria. African Journal of Traditional Complementary and Alternative Medicine 4(2):191-198. doi: 10.4314/AJTCAM.V4I2.31207

Ojo OE, Oyekunle MA, Ogunleye AO, Otesile EB. 2009. E. coli 0157:H7 in food animals in part of S/Western Nigeria: Prevalence and in vitro antimicrobial susceptibility. Tropical veterinarian 26 (3 \& 4):23-30. https://scholar.google.com/citations?user=DTDK8LoAAAAJ\&hl=en (Accessed 6/7/2021)

Oke JM, Oladosun B, Okunola MC. 1999. Sweet potato (Ipomea batatas) tuber - potential oral anti-diabetic agent. African Journal of Biomedical Research 2(1):13-17. https://www.ajol.info/index.php/ajbr/article/view/140557 (Accessed 3/8/2021).

Okonko IO, Soleye FA, AmusanTA, Ogun AA, Udeze AO, Nkang AO, Ejembi J, Faleye TOC. 2009. Prevalence of malaria Plasmodium in Abeokuta, Nigeria.Malaysian Journal of Microbiology 5:113-8. doi:10.21161/MJM.16509.

Okpekon T, Yolou S, Gleye C, Roblot F, Loiseau P, Bories C, Grellier P, Frappier F, Laurens A, Hoquemiller R. 2004. Antiparasitic activities of medicinalplants used in Ivory Coast. Journal of Ethnopharmacology 90: 91-97. doi: 10.1016/j.jep.2003.09.029.

Okujagu TF. 2005. Welcome address at the Zonal training for traditional medicine practitioners, Port- Hacourt, Nigeria. Nigeria Natural Medicine Development Agency, Federal Ministry of Science and Technology.

Olabinrin BM, Eniyansoro OO, Okoronkwo CO, Olabinrin PF, Olaleye MT.2010. Evaluation of chelating ability of aqueous extract of Tetracarpidium conophorum (African walnut) in vitro. International Journal of Applied Research in Natural Products 3(3):13-18. https://journaldatabase.info/articles/evaluation_chelating_ability_aqueous.html (Accessed 3/7/2021).

Oladeji O. 2016. The characteristics and roles of medicinal plants: some important medicinal plants in Nigeria. Natural Products: An Indian Journal 12 (3):102. https://www.tsijournals.com/articles/the-characteristics-and-rolesof-medicinal-plants-some-important-medicinal-plants-in-nigeria.html (Accessed 3/8/2021).

Oladunmoye MK, Kehinde FY. 2011. Ethnobotanical surveyof medicinal plants used in treating viral infections among Yoruba tribe of southwestern Nigeria. African Journal of Microbiology Research 5(19): 2991-3004. doi: 10.5897/AJMR10.004.

Olanrewaju O, Akanni OL. 2010. Health expenditure and health status in Northern and Southern Nigeria: a comparative analysis using NHA framework. Paper presented the 2010 CSAE conference held at St. Catherine College, University of Oxford, Oxford, UK.

http://citeseerx.ist.psu.edu/viewdoc/download?doi=10.1.1.192.6678\&rep=rep1\&type=pdf (Accessed 9/12/2020).

Olorunnisola OS, Adetutu A, Balogun EA, Afolayan AJ. 2013. Ethnobotanicalsurvey of medicinal plants used in the treatment of malaria in Ogbomoso, South-west, Nigeria. Journal of Ethnopharmacology 150: 71-78. doi: 10.1016/j.jep.2013.07.038.

Olorunniyi OF, Morenikeji OA. 2013. The extent of use of herbal medicine inmalaria management in Ido/Osi Local Government Area of Ekiti State, Nigeria. Journal of Medicinal Plants Research 7(42):3171-3178. doi: 10.5897/JMPR2013.5101.

Olowokudejo JD, Kadiri AB, Travih VA. 2008. Ethnobotanical survey of herbal markets and medicinal plants in Lagos, Nigeria. Ethnobotanical Leaflets 12:851-65. https://opensiuc.lib.siu.edu/ebl/vol2008/iss1/116 (Accesssed 3/8/2021).

Omoregie ES, Pal A, Sisodia B. 2011. In vitro antimalarial and cytotoxic activitiesof leaf extracts of Vernonia amygdalina (Del.). Niger. Journal of Basic and Applied Sciences 19(1):121-126. doi: 10.4314/njbas.v19i1.69356.

Omoruan Al, Bamidele AP, Phillips OF. 2009. Social Health Insurance and sustainable health reforms in Nigeria. EthnoMedicine 3:105-10. doi:10.1080/09735070.2009.11886346. 
Onwujekwe OE, Obikeze EN, Ogonnia GO, Onoka CA. 2010. Investigating determinants of out-of-pocket spending and strategies for coping with payments for health care in southeast Nigeria. BMC Health Services Research 10(67):1472-6963. doi:10.1186/1472-6963-10-67.

Periera JA, Oliveira SA, Valentao P, Andrade PB, Ferreira IC, Ferreres F. 2007. Walnut (Juglans regia L.) polyphenolic compounds, antibacterial activity and antioxidant potentials of different cultivars, Food and Chemical Toxicology 45(11):2287-2295. doi: 10.1016/J.FCT.2007.06.004.

Peters RH. 1991. A critique for Ecology. Cambridge University Press, New York, U.S.A.

Rajendra P, Sapna J, Shraddha K, Ajay K. 2012. Properties and therapeutic application of bromelian: A review. Biotechnology Research International 2012:1-6. doi: 10.1155/2012/976203.

Razalia N, Razaba R, Junita SM, Aziz AA. 2008. Radical scavenging and reducing properties of Cashew shoots (Anacardium oocidentale). Food Chemistry 111:38-44. doi: 10.1016/J.FOODCHEM.2008.03.024.

Ryan J, 2018. Boards and Beyond: infectious Disease. Create Space Independent Publishing Platform, U.S.A.

Savikin K, Zdunic G, Menkovic N, Zivkovic J, Cujic N, Terescenko M, Bigovic D. 2013. Ethnobotanical study on traditional use of medicinal plants in SouthWestern Serbia, Zlatibor district, Journal of Ethnopharmacology 146:803-10. doi: 10.1016/j.jep.2013.02.006.

Soladoye MO, Chukwuma EC, Sulaiman OM, Feyisola RT. 2014. Ethnobotanical survey of plants used in the traditional treatment of female infertility in southwestern Nigeria. Ethnobotany Research and Applications 12:081090. doi:10.17348/ERA.12.0.081-090.

Soladoye MO, Lewis GP. 2003. A checklist of Nigerian Legumes. CENRAD Natural Resources Research Assessment and Conservation Series 03, Ibadan, Nigeria, West Africa.

Tantchou TPK, Aldivo J. 1986. Studies on Cameroonian medicinal plants 1: antimalarial activity of the extracts of Alstonia boonei and Guibourtia tessmanii on the Vietnam Smith strain of Plasmodium falciparum. Revue Scientifique et Technique Office International Des Epizooties III (3 and 4):69-77.

Tardio J, Pardo-De-Santayuanna M, Morales R. 2006. Ethnobotanical review of wild edible plants in Spain. Botanical Journal of the Linnean Society 152:27-71. doi: 10.1111/J.1095-8339.2006.00549.X.

Tona L, Cimanga RK, Mesia K, Musuamba CT, De Bruyne T, Apers S, HernansN, Van Miert S, Pieters L, Totte J, Vlietinck AJ. 2004. In vitro antiplasmodial activity of extracts and fractions from seven medicinal plants used in the Democratic Republic of Congo. Journal of Ethnopharmacology 93:27-32. doi: 10.1016/j.jep.2004.02.022.

Traore F, Gasquet M, Laget M, Guiraud H, Di-Giorgio C, Azas N, Doumbo O,Timon-David P. 2000. Toxicity and genotoxicity of antimalarial alkaloid rich extracts derived from Mytragyna inermis $\mathrm{O}$. Kuntze and Nauclea latifolia. Phytotherapy Research 14:608-611. doi: 10.1002/1099-1573(200012)14:8<608:aid-ptr667>3.0.co,2-d.

Treasure IO, Adjene JO, Odigie MO. 2020. Ethnobotanical survey of medicinal plants in Ughelli North Local Government Area of Delta State. Journal of Medicine: Study and Research 3(14):1-9. doi: 10.24966/MSR$5657 / 100014$.

Ubom RM. 2010. Ethnobotany and biodiversity conservation in the Niger Delta, Nigeria. International Journal of Botany 6(3):310-322. doi:10.3923/JJB.2010.310.322.

Udeinya IJ, Brown N, Shu EN, Udeinya FI, Quakeyie I. 2006. Fractions of an antimalarial neem-leaf extract have activities superior to chloroquine and are gametocytocidal. Annals of Tropical Medicine and Parasitology 100:1722. doi: $10.1179 / 136485906 \times 78508$.

Ugbogu OA, Chukwuma EC. 2019. Ethnobotany of Okomu Forest Reserve, Edo, State, Nigeria. Journal of Applied Sciences and Environmental Management 2397:1391-1401. doi: 10.4314/jasem.v23i7.31.

Uzochukwu BSC, Ughasoro MD, Etiaba E, Okwuosa C, Envuladu, E, Onwujekwe OE. 2015. Health care financing in Nigeria: Implications for achieving universal health coverage. Nigerian Journal of Clinical Practice 8(4):437-444. doi: 10.4103/1119-3077.154196.

Wambebe C. 1998. Development of standardized phytomedicines. African Journal of Pharmaceutical Research and Development 3:1-11. 
WHO. 1978. Alma Ata Declaration Primary Health Care. Health for all Series No. 1.

WHO. 1978. The promotion and development of traditional medicince. Technical Report No.622, Geneva, Switzerland.

Zheng X, Xing F. 2009. Ethnobotanical study on medicinal plants around Mt. Yinggeling, Hainan Island, China. Journal of Ethnopharmacology 124:197-210. doi: 10.1016/j.jep.2009.04.042. 